\title{
Fragmentation Versus Cohesion
}

\author{
Emmanuel Villermaux ${ }^{1,2}$ \\ ${ }^{1}$ Aix Marseille Université, CNRS, Centrale Marseille, IRPHE, Marseille, France \\ ${ }^{2}$ Institut Universitaire de France, Paris, France
}

(Received xx; revised xx; accepted $\mathrm{xx}$ )

Capillarity is the familiar manifestation of the cohesion of liquids. Since Laplace (1805), we know that intense attractive forces between the molecules bridge the small with the large as they shape liquid/vapor interfaces at the macroscopic scale through the concept of surface tension (menisci, drops, bubbles, puddles, liquid rise in tubes, etc...). We concentrate on situations where liquids 'disgregate', following the neologism of R. Clausius (1862), meaning that they fragment by the action of deformation stresses whose intensity competes with that of cohesion forces. Various examples, including explosions, blow-ups, hard and soft impacts, and shears applied to liquid jets, sheets and drops are reviewed. They concern applications ranging from liquid propulsion, agricultural spraying, to the formation of ocean spray, raindrops, and human exhalations by violent respiratory events. In spite of their diversity, the various modes of fragment production share an ultimate common phenomenology -the ligament dynamics-, suggesting that the final stable droplets size distribution can be interpreted from elementary principles.

\section{CONTENTS}

\section{Overview: issues and scope}

1.1. Objects of interest and scope 3

1.2. Distributions rather than just averages 3

1.3. A fragmented world 5

2. Cohesion, Aggregation and Fragmentation $\quad 5$

2.1. Founding idea of Laplace (1805) 5

2.2. Clausius disgregation (1862) 7

2.3. Fragmentation of a necklace of magnets $\quad 7$

2.4. Outline 10

3. Static and dynamic equilibria, classical results 11

3.1. Shapes of liquid volumes at rest: bubbles, films, minimal surfaces, puddles, menisci, capillary ascent, capillary length

3.2. Dynamic equilibria: Film edge recession, Taylor-Culick formulae, paradox, holes, Savart sheets and bells 13

4. Rupture of sheets and films 20

4.1. Phenomena at the rim: cusps 20

4.2. Spontaneous hole formation 23

4.3. Kinematic thinning 26

4.4. 'Effervescent' Atomization 28

5. Shells 29

5.1. Exploding bubbles and drops 29

5.2. Singular Savart Bell $\quad 32$

5.3. Surface bubbles $\quad 32$

6. Gone with the wind: shear, flags, spray and exhalations 34

6.1. Shear at the interface $\quad 35$ 
6.2. Viscoelastic fluids 36

6.3. Thin films: flapping 36

$\begin{array}{ll}\text { 6.4. The wavy corridor mechanism } & 37\end{array}$

7. Impacts 38

7.1. Hard impacts 39

$\begin{array}{ll}\text { 7.2. Soft impacts } & 45\end{array}$

7.3. Chemical synthesis by impact $\quad 47$

8. Jets, threads, smooth ligaments $\quad 47$

8.1. Production of jets 48

8.2. Mode selection on a varying substate $\quad 52$

9. The post Plateau-Rayleigh era: Corrugated ligaments 53

9.1. Paradigm of the corrugated ligament 54

9.2. Inverse cascade of aggregations: lessons from a necklace of magnets $\quad 54$

9.3. Rough ligaments: rearrangements versus instability timescales $\quad 57$

9.4. Blobs on a ligament: fractional convolutions, Gamma distributions $\quad 60$

$\begin{array}{ll}\text { 9.5. Dissection of a jet } & 64\end{array}$

$\begin{array}{ll}\text { 9.6. Compound distributions } & 66\end{array}$

10.Formation of 'fines', direct cascades 71

$\begin{array}{ll}\text { 10.1. Iterated thread fission, coalescence cascade } & 72\end{array}$

$\begin{array}{ll}\text { 10.2. Imploding cavities } & 73\end{array}$

10.3. Collision of rims $\quad 73$

$\begin{array}{ll}\text { 10.4. Reactive droplets, fireworks } & 74\end{array}$

$\begin{array}{ll}\text { 10.5. Minimal fragment size } & 74\end{array}$

$\begin{array}{ll}\text { 11.Energetics of fragmentation } & 75\end{array}$

$\begin{array}{ll}\text { 11.1. Misconceptions } & 75\end{array}$

$\begin{array}{ll}\text { 11.2. Efficiency } & 77\end{array}$

$\begin{array}{ll}\text { Appendix A } & 78\end{array}$

$\begin{array}{ll}\text { A.1. Rayleigh Taylor } & 78\end{array}$

$\begin{array}{ll}\text { A.2. Keller Kolodner } & 79\end{array}$

A.3. Helmholtz Kelvin Rayleigh $\quad 81$

$\begin{array}{ll}\text { A.4. Squire } & 82\end{array}$

A.5. Plateau Rayleigh Weber $\quad 83$

\section{Overview: issues and scope}

In his treatise on Cohesion, J. S. Rowlinson notes that Some problems have always been with us. Among them notably: Why does matter stick together (Rowlinson 2002)? We could, identically, ask the same question concerning fragmentation: Why does condensed matter break? After all, even if stressed, sheared, compressed or stretched, a piece of cohesive matter could well remain as a whole rather than splitting into pieces; and it actually does so when perturbed sufficiently gently. But then, what does gently means? And when a stressed object breaks, why is it typically into many pieces, with many more small than bigger ones, rather than just in two pieces (think of a glass dropped on the floor)? Is there a way to quantify the diversity of the sizes? Is there a minimum fragment size, or can the object divide ad-infinitum? Why is it that a liquid volume may fragment spontaneously (like a jet), or on the contrary keeps its integrity (like a puddle lying on a solid surface) depending on its shape only?

Answers to some of the above questions exist, the others are still under debate and are 


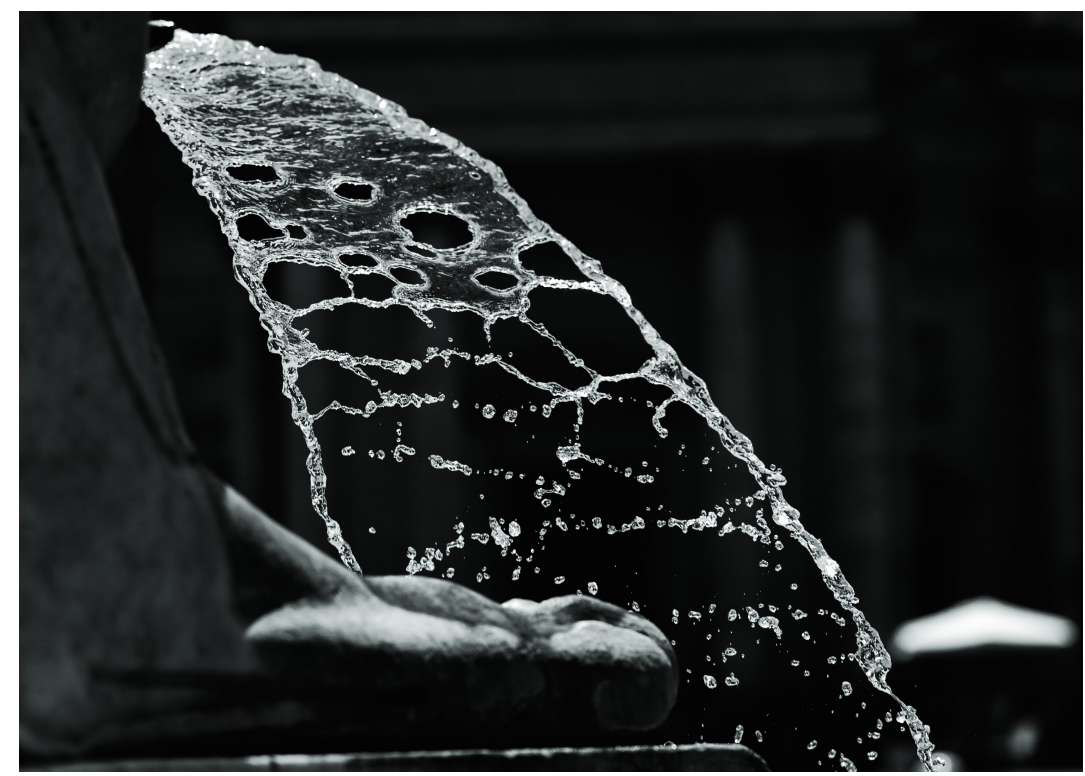

Figure 1. Rome, Piazza del Popolo, the lions of the central fountain expectorate a turbulent water sheet. Random velocity fluctuations and possible bubbles inclusions in the liquid nucleate holes which, driven by surface tension, grow and merge, leaving the sheet as a set of connected ligaments. These corrugated ligaments further breakup into a collection of disjointed droplets broadly distributed in size.

the subject of active research in Fluid Mechanics and beyond. Their study is the topic of the present review.

\subsection{Objects of interest and scope}

The subject matter, illustrated in Fig. 1, will be concerned with liquid sheets, shells, holes, jets and ligaments, those which ultimately break into stable fragments, namely the final drops. Since these ligaments, kind of elongated, finite sized rough columns will be shown to be the 'sinews' of liquid fragmentation (Villermaux 2007), their formation will be tracked, and described along with the instabilities giving birth to them in a variety of different situations with applications ranging from liquid propulsion, agricultural spraying, to the formation of ocean spray, raindrops, and human exhalations by violent respiratory events etc... It will be shown that their dynamics, and final outcome, results from a trade-off between cohesion and fragmentation.

Attention will be paid in each case to understanding the overall drop size distribution, for at least two reasons: First because a satisfactory understanding of a physical phenomenon requires its complete statistical description, particularly when it is non-trivial, and second because most applications urge us to do so, as we explain next.

\subsection{Distributions rather than just averages}

We list below a number of situations taken from various fields showing the importance, in fragmentation, to understand or control the overall drops size distribution, rather than just a mean size.

(i) Liquid propulsion, combustion, drying: Fragmentation is a mandatory step in various industrial processes like in furnaces, liquid propulsion engines, or drying facilities, contexts where it is usually called atomization (i.e. literally, down to the atom size). 
Although produced in a swarm with other droplets densely packed in space at the atomization nozzle, it is nevertheless often the fate of an individual liquid droplet which is of practical interest: the length of a liquid-propulsion combustion chamber, or the height of a drying tower depend on the distance from the injection nozzle for the last, biggest droplet of fuel, or solvent, to evaporate, burn (Marble 1964), or solidify (Yule \& Dunkley 1994). Conversely, fine droplets burn too soon after their formation, and may damage the injectors. A better control of the fuel droplet size distribution at injection is one of the ways to improve car engines consumption (Taylor 2008).

(ii) Agricultural spraying, snow lances, painting and printing industry, dust control: Spray drift is a major concern in agriculture. Standard flat fan atomizers used to spray fields with fertilizers and pesticides produce broad drops sizes distributions, with a notable fraction with diameter below $100 \mu \mathrm{m}$ (called 'fines') likely to be swept by the wind, reaching the farmer's neighbor field who may not like it, or the river next to it (Hewitt 2000; Kooij et al. 2018). Strategies to reduce their relative number are the subject of active research (Hilz et al. 2012; Vernay et al. 2015a). The biggest droplets however soon fall by their own weight, are too heavy to hook on plants leaves, or rebound on them, splash like raindrops do, favoring plant-to-plant contamination (Gilet \& Bourouiba 2015). Snow lances, whose popularity is indexed on the progress of global warming, suffer from the same flaw: light snowflakes from tiny droplets wander aloft, missing the targeted ski slope, while big droplets fall on the ground before freezing, finally producing undesired ice. For similar reasons, the rotary atomizers used in the automotive painting industry produce many droplets that fail reaching the car bodies they are supposed to coat (Wilson et al. 2018). Dust particles are routinely removed from air by sprays with a capturing efficiency depending critically on the droplets sizes (Swanson \& Langefeld 2015). The formation of undesired small satellite droplets causes notorious difficulties in inkjet printing (Wijshoff 2010; Basaran et al. 2013).

(iii) Geophysics, planetary sciences, precipitations: The Earth was built by high-energy impacts of planetesimals with metallic cores of their own. The composition of the Earth mantle depends critically on the time offered to metal-silicate chemical equilibration as the impactor material settles towards the Earth core. It fragments very much like raindrops form as they fall from clouds in the atmosphere (Villermaux \& Bossa 2009), producing broad size distributions (Marshall \& Palmer 1948). Efficient equilibration is achieved with smaller fragments allowing fast metal-silicate mass transfer, while the fate of bigger ones is to accumulate in the -therefore iron-rich-core. The overall planet chemical composition relies on fragmentation (Deguen et al. 2014; Landeau et al. 2014; Wacheul et al. 2014).

(iv) Sea spray: The spray produced by wave breaking in the surf zone, or white caps at the ocean surface is a superposition of different mechanisms which results in broadly distributed droplets sizes (nanometers to millimeters, see e.g. O'Dowd \& de Leeuw (2007); Veron (2015)). If they all contribute to the global air-sea exchanges, the fate of each droplet in this distribution is not identical: The smallest aerosols are long lived in the atmosphere, carry salt and diverse chemical/biological substances (Cochran 2017; Pietsch et al. 2018) over large distances inland while the biggest spume droplets carry momentum, heat and produce moisture by evaporation, feeding hurricanes for example, before settling by gravity.

(v) Exhalations, disease transmission, seeding: Human exhalations like coughing or sneezing produce a broad spectrum of droplets sizes, possibly carrying pathogens and thus mediate disease transmission (Pasteur 1861; Flügge 1897; Winslow \& Robinson 1910; Duguid 1946). This well known fact is currently re-examined in the light of modern methods and ideas (Turner et al. 1941; Bourouiba et al. 2014). The contamination radius 
of an infected coughing individual is the distance needed for the last liquid droplet exhaled from his mouth and staying aloft in air to evaporate (Wells 1955), but it is not clear which are the nastiest most long-lived droplets in the exhaled spectrum; It has been suggested that the finest the nastiest, through a collective evaporation delay mechanism (Villermaux et al. 2017). Fungi reproduce by ejecting encapsulated seeds of different sizes, the biggest, heaviest and thus farthest reaching ones being responsible for substrate exploration, while the smallest exploit the substrate in the immediate emitter vicinity (Ingold 1971). These airborne spores, like pollens, can be carried over long distances, crossing oceans (Hirst et al. 1967; Aylor 2017).

(vi) Medicine, forensic science, inhaled drugs, scents: Some therapies rely on inhaled aerosols carrying drugs which should be embedded in particles whose size is critical to escape from the lung's natural clearance mechanisms. Controlling particles sizes, and density is vital in this context (Edwards et al. 1997). Experts in the forensic technique of Blood Pattern Analysis routinely contemplate blood splats to decipher the circumstances of a homicide. These splats consist of myriads of stains on the floor which are meaningful only when the fragmentation process creating them has been understood (Attinger et al. 2013). Electronic cigarettes (e-cigs) are efficient, cancer agent free, tobacco smoking alternatives. However, the nicotine-rich aerosol produced by e-cigs has a droplet size distribution appreciably more skewed towards small sizes than the tobacco smoke particles, with a fat tail towards 'nano' particles; they are, for this reason, considered as suspect (Glantz \& Bareham 2018). There is a, yet unexplored, relationship between the way a fragrance spray has been atomized, and the persistence of its scent, admittedly a fascinating question.

\subsection{A fragmented world}

The examples above suggest that we are indeed living in a fragmented world, in more than one sense. There are other sectors of science where the dilemma between cohesion and fragmentation has been identified as paramount: For example, the survival of colonies of living species is known to be influenced by their habitat fragmentation. While fragmentation is usually believed to have a deterring impact on wildlife colonies because it promotes species competition for limited resources and inbreeding, thus hampering bio-diversity (Betts 2019), other aspects like long lasting adaptation to a given small perimeter substrate in an immigration/emigration-free isolation is known to favor persistence (Mc Arthur \& Wilson 1967; Letcher et al. 2007). In sociology, spatial fragmentation and its cultural corollary have been identified to impede social cohesion, and are considered as a threat to democracy. These topics are however out of the scope of this review.

\section{Cohesion, Aggregation and Fragmentation}

Before we wonder how cohesive objects break, it is useful to recall in which manner they have been understood to be, precisely, cohesive.

\subsection{Founding idea of Laplace (1805)}

The founding idea is due to Laplace (1805). Liquids rise spontaneously in narrow tubes. Well aware of Newton's Query 31 (Newton 1704), and of the subsequent experimental observations by Hawksbee and Jurin intended to solve this inadmissible exception to the theory of gravitation (see the detailed history in Rowlinson (2002)), Laplace conjectured, a century later, the existence of a central force between nearby 'molécules' (or 'particles' 

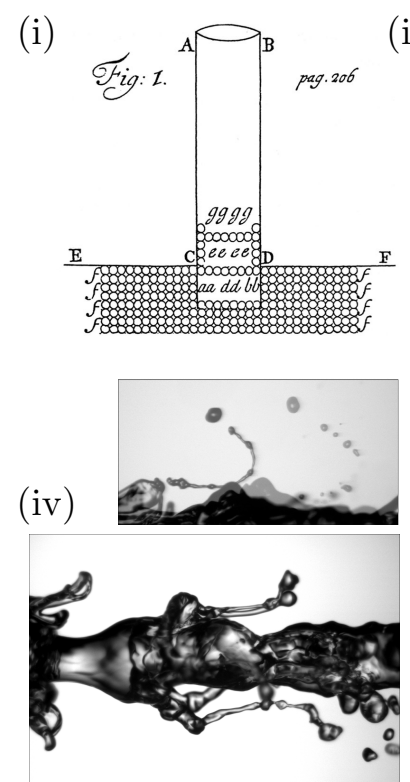

(ii)
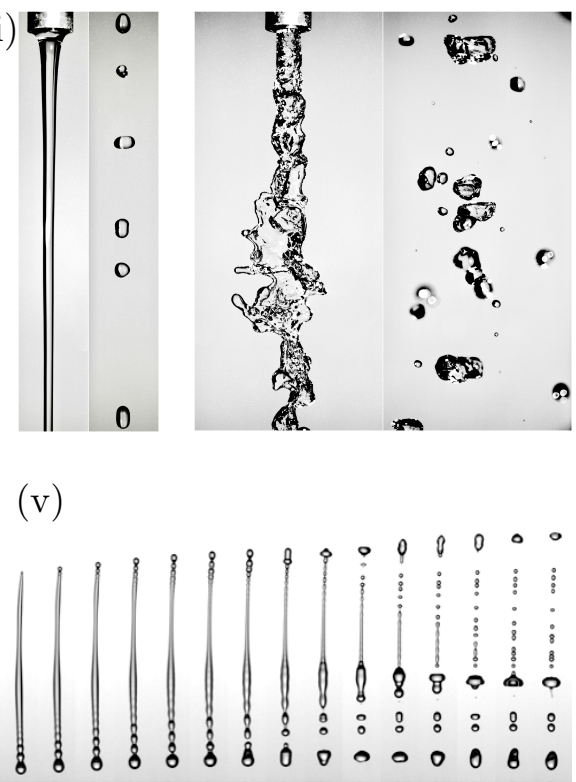

(iii)

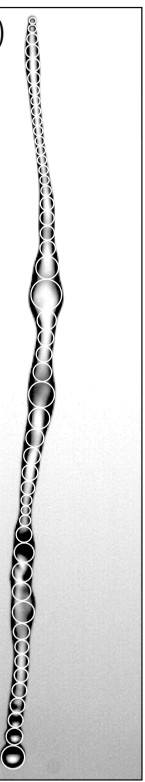

Figure 2. (i) A sketch from Hawksbee (1709) where 'particles' are clearly singled-out as the constitutive components of a liquid, here ascending in a capillary tube. (ii) Breakup of a laminar, falling smooth water jet $\left(h_{0}=4 \mathrm{~mm}\right.$ and $\left.v_{0}=1 \mathrm{~cm} / \mathrm{s}\right)$ contrasted with the fragmentation of the same, but turbulent jet. (iii) The 'particulate' vision of the rearrangements occurring during ligament breakup (Villermaux et al. 2004). (iv) Stripping of ligaments at the surface of a water jet sheared by a fast coaxial stream (Marmottant \& Villermaux 2004b). (v) The time-lapse of an isolated ligament featuring longitudinal rearrangements as it breakup.

as Hawksbee called them; this word has to be understood as a synonym of small amount, for instance, Newton (1687) refers to a particle of time to designate an infinitesimal timeincrement): Two masses $m$ and $m^{\prime}$ distant by $f$ are attracted by a force $m m^{\prime} \phi(f)$ with $\phi(f)$ assumed to be very intense at short distance, and evanescent for large separation distances $f$, in Laplace's notations. This formalism, exposed in his Traité de Mécanique Céleste is directly imported from the one operating for gravitational forces. The work of separation, or binding energy of the particles pair is $u(f)=\int_{f}^{\infty} \phi\left(f^{\prime}\right) d f^{\prime}$. Lord Rayleigh (1890), in his exegesis of Laplace's contribution, shows how successive summations provide the attraction from a half space with density $\rho$ on a unit mass at distance $z$ from its bounding plane $\psi(z)=2 \pi \rho \int_{z}^{\infty} f u(f) d f$, that the mutual attraction between two half spaces separated by $x$ is $\theta(x)=\rho \int_{x}^{\infty} \psi(z) d z$ per unit plane surface area, producing an internal bulk pressure $K=\theta(0)$, and that the separation work of the two planes from contact is $2 \sigma=\int_{0}^{\infty} \theta(x) d x$.

Thus defined as an energy per unit surface, what is now called the surface tension of the material or, equivalently, its fracture energy (we will come back to this delicate question in Section 11), is related to the hypothesized microscopic force $\phi(f)$ by the celebrated formulae

$$
\sigma=\frac{\pi}{8} \rho^{2} \int_{0}^{\infty} f^{4} \phi(f) d f
$$

Surface tension is a finite quantity provided the force $\phi(f)$ decays fast enough with $f$, and it is not the least of Laplace talents to have embarked in this description without, at the time, an empirical proof of the existence of molecules and ignoring that, more than a century later, quantum mechanics would rationalize the so-called van der Waals 
forces (Israelachvili 1991) by showing that $u(f) \sim f^{-6}$ and that therefore $\phi(f) \sim f^{-7}$, a decay making the integral in (2.1) convergent indeed (Laplace had envisaged, safely, an exponential decay).

\subsection{Clausius disgregation (1862)}

Clausius (1862) created the ephemeral concept of disgregation which he himself soon replaced by the notion of entropy. Clausius wanted to formalize the idea that a body mass exchanging heat or work with an outside source suffers an 'alteration of arrangement' of the 'aggregation state' of its constitutive molecules:

... the effect of heat always tends to loosen the connexion between the molecules, and so to increase their mean distances from one another. In order to be able to represent this mathematically, we will express the degree in which the molecules of a body are dispersed, by introducing a new magnitude, which we will call the disgregation of the body...

We would nowadays call disgregation the configurational part of the entropy but it is interesting to note that by 'dispersed', Clausius equally meant loosely aggregated (like molecules in a liquid), or completely disjointed (like in a gas). Cohesion, aggregation and fragmentation are thus notions which were thought at this time as parts of the same problem, or at at least as having strong inter-relationships.

We have on purpose recalled the early 'particulate' description of matter cohesion first to underline its origin as an inspiration from the theory of gravitation and central forces between isolated bodies. It is also possible that Laplace, who knew well the experimental background of the Cambridge group, notably the one of Hawksbee, has been influenced by one of his drawings, reproduced in Fig. 2, where sizable 'particles' are clearly evidenced as the constitutive components of a liquid. And second, because this representation will be useful to understand the dynamics of fragmentation per-se, and the origin of the multiplicity in fragments sizes, through considerations à la Clausius where disgregation and aggregation compete on an unstable substrate (Section 9). We examine such an example below.

\subsection{Fragmentation of a necklace of magnets}

In order to see how cohesion and disgregation compete, it is instructive as a starting exercise to work-out the caricatural problem of the sudden forced radial expansion of an assembly of attracting particles arranged in a circle: the shatter of necklace of magnets (Vledouts et al. 2015, 2016b).

A set of spherical magnets is assembled in a necklace positioned on a cone with an incline at $45^{\circ}$. The cone, guided by an axle, is released from rest at a given height and when it hits the ground, it is suddenly stopped. An initial impulse in the radial direction is communicated to each sphere, and the necklace expands at radial speed $V$. Consequently, the spheres separate from each other; the necklace is literally 'atomized'. But, because of the attractive force linking them, the spheres start concomitantly to aggregate in bigger clusters, a process going on at an ever slowing down pace to finally stop when the clusters are too heavy, and distant from each other to aggregate. Fragments of various sizes are then irreversibly formed (the collision between clusters is inelastic, with virtually no rebound, see Hinch \& Saint-Jean (1999) for the opposite situation). The sequence of events, with the corresponding inverse cascade of aggregations is illustrated in Fig. 3. 
(i)
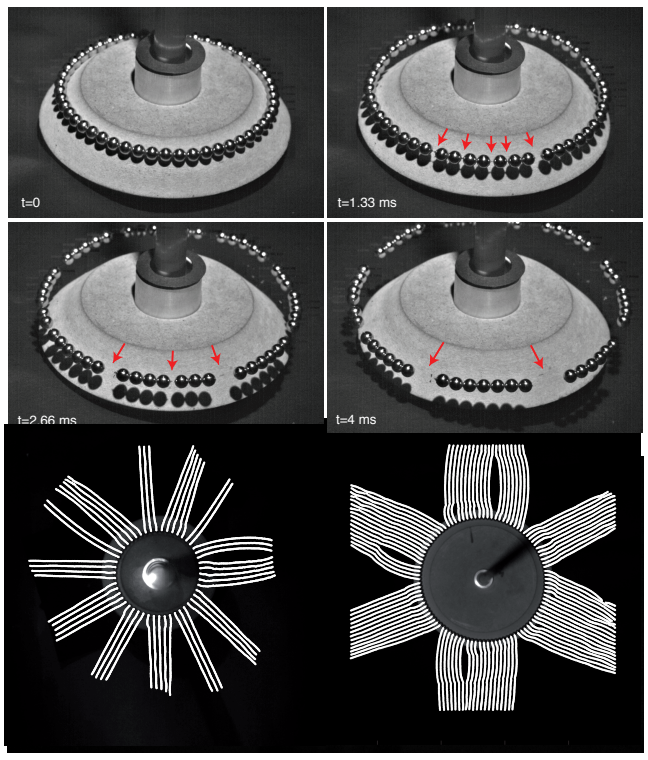
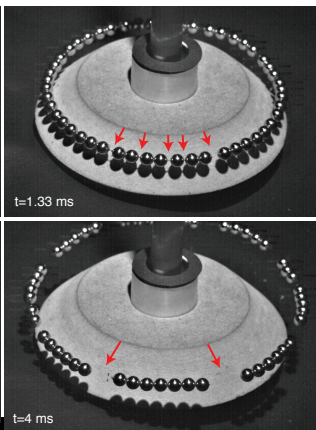

(a)

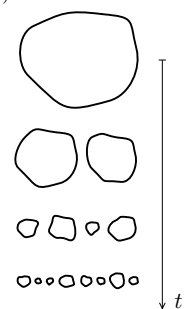

(ii)

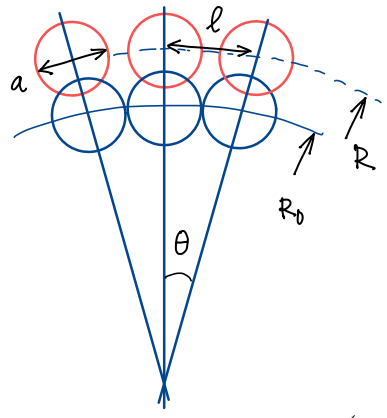

(b)

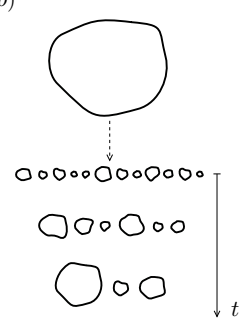

Figure 3. Fragmentation of a necklace of spherical magnets (Vledouts et al. 2015, 2016b). (i) A ring made of spherical magnets of diameter $a=5 \mathrm{~mm}$, mass $m=0.5 \mathrm{~g}$ and mutual contact force $\phi(a)$ is placed on a cone, which is let fall on the ground. At impact, each sphere is communicated a radial speed $V$ and the ring expands. The spheres separate from each other and start to aggregate, driven by the attractive magnetic force. The arrows indicate intervals between fragments. (ii) Sketch of the separation process. (iii) (a) The conventional direct, sequential cascade of breakups for which the arrow of time points towards ever smaller fragments sizes. (b) The inverse cascade of aggregations, occurring after the smallest sizes accessible by the system have been formed in a first step (dotted arrow). Fragments sizes get larger as time proceeds.

\subsubsection{Radial deceleration}

The central force between two spherical particles of diameter $a$, of magnetization density $M$ separated by a centre-to-centre distance $\ell$ is

$$
\phi(\ell)=\phi(a) \times\left(\frac{a}{\ell}\right)^{4}
$$

with $\phi(a)=\pi \mu_{0} M^{2} a^{2} / 24$ where $\mu_{0}$ is the vacuum permeability (Jackson 1998). Spheres are essentially attracted by their nearest neighbors, and the first consequence of this triplet interactions along the curved necklace shape is the decrease of the expansion radial speed. Attractive forces on a curved substrate induce a Laplace, surface tension force decelerating the cohesive necklace. If $R$ is the necklace radius and $R(t=0)=R_{0}$, its equation of motion writes

$$
\begin{aligned}
m \ddot{R} & =-\phi(R \theta) \times 2 \sin (\theta / 2) \\
\theta & =a / R_{0} \ll 1
\end{aligned}
$$

where $m$ is the sphere mass, integrating into

$$
\dot{R}^{2}=V^{2}-V_{c}^{2}\left(1-\left(\frac{R_{0}}{R}\right)^{3}\right), \quad \text { with } \quad V_{c}^{2}=\frac{2}{3} \frac{a \phi(a)}{m}
$$

The critical velocity $V_{c}$ is the escape velocity of the process, meaning the velocity below which an initial expansion at velocity $V$ will coalesce back to the initial state $R_{0}$. For 
$V>V_{c}$, the expansion velocity $\dot{R}$ is strongly slowed down at short time, before reaching a steady value, smaller that $V$ as

$$
\left(\frac{\dot{R}}{V}\right)^{2} \underset{t \rightarrow 0}{\longrightarrow} 1-\frac{V_{c}^{2}}{V} \frac{t}{R_{0}}, \quad \text { and } \quad \dot{R}^{2} \underset{R \rightarrow \infty}{\longrightarrow} V^{2}-V_{c}^{2}
$$

This decay and final shift from $V$ reflects the transfer of kinetic energy into potential energy, the one which was spent at breaking the liaisons between the spheres. We will come back to these energetic considerations in Section 11.

\subsubsection{Ortho-radial aggregation}

The other consequence of the central force $\phi(\ell)$ besides expansion slowing down is the concomitant aggregation of the spheres, and then of clusters of spheres. Two neighbor fragments with $p$ and $q$ spheres respectively, separated by a distance $\ell$ between the center of their facing end spheres interact through $\phi(\ell)$, the force between the end spheres. The equation of motion for $\ell$ then reads

$$
\ddot{\ell}=-\frac{\phi(a)}{m}\left(\frac{a}{\ell}\right)^{4}\left(\frac{1}{p}+\frac{1}{q}\right)
$$

whose first integral is again

$$
\dot{\ell}^{2}=u_{p, q}^{2}-\frac{p+q}{p q} V_{c}^{2}\left(1-\left(\frac{a}{\ell}\right)^{3}\right), \quad \text { with } \quad u_{p, q}=V \frac{a}{R_{0}} \frac{p+q}{2}
$$

where $u_{p, q}$ is the geometrical initial divergence velocity (i.e. in the ortho-radial direction) of two clusters of size $p$ and $q$. Making $\zeta=\ell / a$ and $\tau=u_{p, q} t / a$, we have

$$
\frac{d^{2} \zeta}{d \tau^{2}}=-\frac{3}{2} \frac{\epsilon_{p, q}}{\zeta^{4}}
$$

with $\zeta(0)=\dot{\zeta}(0)=1$ and

$$
\epsilon_{p, q}=\left(\frac{u_{c}}{u_{p, q}}\right)^{2}, \quad \text { with } \quad u_{c}^{2}=V_{c}^{2} \frac{p+q}{p q}
$$

The critical velocity $u_{c}$ is the divergence velocity above which two cohesive fragments with sizes $p$ and $q$ will not reconnect. Initially $p=q=1$ and successive cluster-cluster coalescences increase these numbers. Aggregation thus proceeds as long as $u_{p, q}<u_{c}$, or $\epsilon_{p, q}>1$, and the necklace fragmentation is completed as soon as $\epsilon_{p, q}<1$ for all neighboring fragments of size $p$ and $q$. The fragments trajectories described by (2.9), the cluster coalescence timescales, the mean fragment growth and the details of the aggregation cascade are discussed by Vledouts et al. (2015); the role of pre-existing defects along the necklace is considered in Vledouts et al. (2016b).

\subsubsection{The Weber number}

In this necklace shattering example like in all fragmentation problems, disgregation and cohesion compete and cooperate in building the overall fragment size distribution.

There is a number which measures the initial ratio of these two ingredients, namely the Weber number (it was, actually, introduced by Constantin Weber (Weber 1931) not even in the form of a dimensionless quantity, and in the very different context, examined in Section 8, of jet stability, see also Appendix A.5). In our example, fragmentation is completed at the end of the inverse coalescence cascade marked by $\epsilon_{p, q}=1$, a condition 
we will first write for the average fragment size $p=q=\langle n\rangle$, leaving the discussion about the distributions of fragments sizes to Section 9. From (2.10), we see that

$$
\langle n\rangle^{3 / 2} \sim \frac{V_{c}}{V} \frac{R_{0}}{a}
$$

The surface tension $\sigma$ of the necklace is approximately such that $a^{2} \sigma \approx \int_{a}^{\infty} \phi(\ell) d \ell$ for this one-dimensional cohesive object so that $\sigma \approx \phi(a) / a$, and the mass of a sphere with density $\rho$ is $m \sim \rho a^{3}$. Introducing the particle Weber number

$$
W e=\frac{\rho u_{1,1}^{2} a}{\sigma}
$$

where $u_{1,1}=V a / R_{0}$ is the divergence velocity at the scale of the elementary molecule, or particle constitutive of the cohesive necklace, we can re-write $(2.11)$ in terms of $W e$ as

$$
\langle n\rangle \sim W e^{-1 / 3}
$$

This scaling relationship, in which $W e$ can at most be equal to 1 for $\langle n\rangle \gtrsim 1$ can also be viewed as a simple (but flawed, see Section 11) energy balance in which the kinetic energy $\frac{1}{2} \rho\langle n\rangle a^{3} u_{\langle n\rangle,\langle n\rangle}^{2}$ of the diverging motion $u_{\langle n\rangle,\langle n\rangle}=\langle n\rangle u_{1,1}$ equilibrates the cohesion energy $a \phi(a)=a \sigma$. It was derived essentially in this form, and from the above reasoning, in different but related contexts (Grady 1982, 2006; Bazant \& Caner 2013).

The relation (2.13) involves naturally the divergence velocity at the scale $a$ of the molecule and at that scale, $W e<1$ when the necklace is not trivially atomized into single particles with $\langle n\rangle=1$. It is nevertheless customary in many applications to construct the Weber number on the sample lengthscale $R_{0}$, and on the injection, directly controllable velocity $V$ (Lefebvre 1989; Bayvel \& Orzechowski 1993; Ashgriz 2011). This large-scale Weber number $\rho V^{2} R_{0} / \sigma$ is typically very large indeed since

$$
W e=\frac{\rho V^{2} R_{0}}{\sigma}\left(\frac{a}{R_{0}}\right)^{3}
$$

with $a / R_{0} \ll 1$ in a macroscopic sample. Even if we will mostly use the (de-facto much larger than unity) macroscopic Weber number in the sequel, one has to remember that it is the one in (2.12) which is dynamically relevant regarding fragmentation (see in particular Section 11 about energetics).

\subsection{Outline}

We are now equipped with the essential concepts to apprehend fragmentation phenomena. After recalling classical results concerning static and dynamic equilibria in Section 3, we will consider the fragmentation of liquid sheets and films in Section 4 , the case of shells in Section 5, the specific aspects associated with a shear with a gas phase in Section 6 and those with impacts in Section 7. The spontaneous fragmentation of jets and threads will be discussed in Section 8 and the important case of corrugated ligaments will be shown to be the key to understand drops size distributions in Section 9. Direct cascades and the formation of fines will be illustrated in Section 10 before we end by considerations on the energetics of fragmentation in Section 11. The Appendix is a catalogue of instabilities relevant to the discussion. 

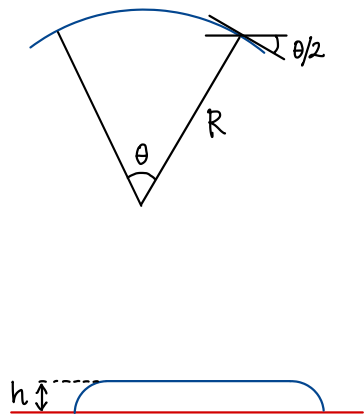
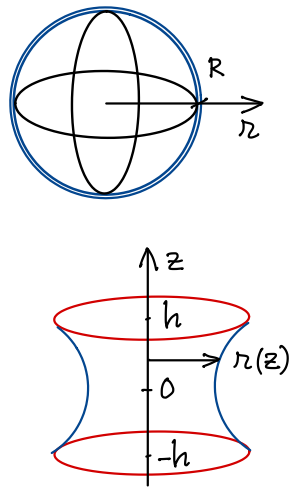

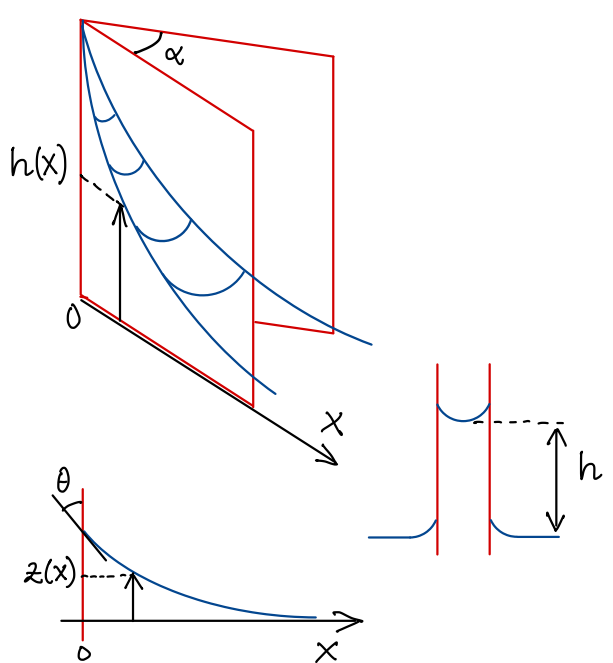

Figure 4. Classical static shapes discussed in Section 3.1. From left to right and top to bottom: The Segner representation of surface tension; A spherical liquid shell of radius $R$; Ascent in a wedge; A puddle lying on a solid floor; A liquid film stretched between two opened rings separated by $h$; A menisci at a solid wall (wetting angle $\theta$ ); Capillary ascent in a tube.

\section{Static and dynamic equilibria, classical results}

In a liquid volume bounded by an interface with principal radii of curvature $R_{1}$ and $R_{2}$ and in view of the construction which has led to (2.1), the pressure $p$ is (Laplace 1805; Maxwell 1875; Lord Rayleigh 1890)

$$
\begin{aligned}
p & =\frac{1}{2} \rho^{2} \int_{0}^{\infty} f^{3} u(f) d f \int_{0}^{2 \pi}\left(\frac{\cos ^{2} \psi}{R_{1}}+\frac{\sin ^{2} \psi}{R_{2}}\right) d \psi \\
& =\frac{\pi}{8} \rho^{2} \int_{0}^{\infty} f^{4} \phi(f) d f\left(\frac{1}{R_{1}}+\frac{1}{R_{2}}\right) \\
& =\sigma\left(\frac{1}{R_{1}}+\frac{1}{R_{2}}\right)
\end{aligned}
$$

which is commonly called Laplace law. It was Laplace's program to compute the shape of static liquid volumes, but this relation shows that the pressure depends solely on the shape of the interface, may it be immobile, or not. We recall below classical results.

3.1. Shapes of liquid volumes at rest: bubbles, films, minimal surfaces, puddles, menisci, capillary ascent, capillary length

The shapes discussed below are all reported in Fig. 4.

\subsubsection{Segner (1751) surface tension}

The energy per unit surface $\sigma$ is also a force per unit length, hence the name surface tension since $\sigma d \mathbf{s}$ is the force acting tangent to the interface perpendicular to the arc length $d \mathbf{s}$ as first suggested by Segner in 1751 (cited by Maxwell (1875), see also Pomeau \& Villermaux (2006)). From there, the structure of Laplace law in (3.3) is readily recovered since, in two dimensions like in our necklace example in Section 2.3, the pressure force $p R \theta$ acting on an interface arc length $R \theta$ is equilibrated by the projection of the surface 
tension force $2 \sigma \sin (\theta / 2)$ perpendicular to the interface, leading to

$$
p=\frac{\sigma}{R}
$$

\subsubsection{Bubble}

Similarly, the surface energy $E$ of a spherical soap bubble can also be computed from the work spent by the pressure force $p(r)=4 \sigma / r$ (since in (3.3) $R_{1}=R_{2}=r$ if $r$ is the current bubble radius, and the bubble shell is bounded by two sides) while inflating it up to its final radius $R$ as

$$
\begin{aligned}
E & =\int_{0}^{R} 4 \pi r^{2} p(r) d r \\
& =2 \times 4 \pi R^{2} \sigma \quad \text { (two sides) }
\end{aligned}
$$

A soap bubble is an example of liquid shells whose fragmentation modes will be studied in Section 5. Under gravity, their structure is constantly evolving, large bubbles are not even spheres, but the spheroidal shape is a good approximation for centimetric bubbles and the reason is that this shape minimizes the surface area (and therefore the surface energy) for a given volume (see the isoperimetric problem in Courant et al. (1996), Ch. VII, $\S 8$ for a simple geometrical justification). It is a nice realization of the principle of least action (see e.g. Isenberg (1978) for a straightforward application to axi-symmetrical shapes).

\subsubsection{Minimal surface}

The catenoid obtained by stretching a film between two circular rings is another celebrated example of an optimal surface. The rings are opened (hence $p=0$ ), their radius is $R$ and they are separated by a distance $2 h$. The radius of the film along the axis of revolution $z$ is $r(z)$ and $r^{\prime}=d r / d z$. The surface energy $E$ and its variation $\delta E$ upon modulations $\delta r$ from the sought optimal shape are

$$
\begin{aligned}
E & =2 \sigma \int_{-h}^{h} f\left(r, r^{\prime}\right) d z, \text { with } f\left(r, r^{\prime}\right)=2 \pi r \sqrt{1+r^{\prime 2}} \\
\delta E & =2 \sigma \int_{-h}^{h}\left\{\frac{\partial f}{\partial r}-\frac{d}{d z} \frac{\partial f}{\partial r^{\prime}}\right\} \delta r d z
\end{aligned}
$$

The optimal shape corresponds to $\delta E=0$ whatever $\delta r$, a condition which cancels the integrand in (3.8), thus providing the Euler-Lagrange equation whose solution is the film shape

$$
\frac{r(z)}{r_{\mathrm{m}}}=\cosh \left(\frac{z}{r_{\mathrm{m}}}\right), \text { and } \frac{R}{r_{\mathrm{m}}}=\cosh \left(\frac{h}{r_{\mathrm{m}}}\right)
$$

a solution (in fact a couple of solutions with one having a lower surface energy), which does exist as long as $h / R \lesssim 0.66$ for $h / r_{\mathrm{m}} \approx 1.2$. The net curvature of the catenoid is indeed zero (Plateau 1873), and Delaunay (1841) has generalized this result to any non-zero constant curvature (like if the sections of the end rims were closed, and the film were either inflated, or deflated with $p \neq 0$ ).

\subsubsection{Puddle, meniscus}

A circular liquid puddle (radius $R$ ) lying on a flat horizontal solid surface is another illustration of the energetic cost associated with interface creation: we assume for simplicity that the liquid is indifferent to the solid, meaning that it has no tendency to wet 
it, or to be repelled by it (the complete problem involves a wetting angle, first introduced by Young (1805), taken here as equal to $\pi / 2$; the subtleties of wetting phenomena are very well discussed in de Gennes (1985), for example). Two kinds of energies compete, the potential energy $\pi R^{2} \int_{0}^{h} \rho g z d z$ of the liquid in the gravitation field if $h$ is the puddle height, and the surface energy $\pi R^{2} \sigma$. For a given puddle volume $\Omega=\pi R^{2} h$, the puddle height $h$ makes the net energy $E$

$$
\frac{E}{\Omega}=\frac{1}{2} \rho g h+\frac{\sigma}{h}
$$

minimum $\left(\partial E / \partial h=0, \partial^{2} E / \partial h^{2}>0\right)$, when

$$
h=\sqrt{\frac{2 \sigma}{\rho g}} \equiv a
$$

where $a$ is called the capillary lengthscale (Landau \& Lifshitz 1987). It is, for the same reason, this lengthscale which sets the typical dimensions of the liquid meniscus wetting a vertical solid wall at an angle $\theta$. The expression of the meniscus profile $z(x)$ is complicated (see e.g. Landau \& Lifshitz (1987); de Gennes et al. (2004)), but the maximal elevation of the meniscus above the flat interface level far from it $(x \gg a)$ is given by $h=a \sqrt{1-\sin \theta}$ while, when $\theta \lesssim \pi / 2$, one has $z(x) \approx h e^{-x / a}$; the meniscus is localized indeed.

\subsubsection{Capillary ascents}

Menisci are one example of the famous shapes, which had remained paradoxical before he rationalized them, solved by Laplace. Others are the ascent of a wetting liquid in the sharp interstice between two plates forming a dihedral (Taylor 1712; Bouasse 1924; Higuera et al. 2008), whose profile is $z(x) \sim a^{2} /(\alpha x)$ with $\alpha$ the angle of the dihedral, and $x$ the distance from its apex, and of course the celebrated capillary ascent in a narrow tube: The weight of the liquid column $\rho g h \pi r^{2}$ is balanced by the capillary force $2 \pi r \sigma \cos \theta$ at the capillary circumference, if $r$ is its radius, and $\theta$ the wetting angle. The liquid elevation is given by Jurin's law (Jurin (1719), who quantified the inverse relationship $h \sim r^{-1}$ )

$$
\frac{h}{a}=\frac{a}{r} \cos \theta
$$

and is equal to zero when the liquid is indifferent to the solid (i.e. $\theta=\pi / 2$ like for water on a surface of silanized glass).

\subsection{Dynamic equilibria: Film edge recession, Taylor-Culick formulae, paradox, holes, Savart sheets and bells}

Equilibrium does not mean absence of motion. Capillary forces may be balanced by inertial and viscous forces, as in the situations we describe below.

\subsubsection{Rim recession: straight edges}

Liquid sheets extruded through slits are bordered by a rim, which has its own dynamics. Holes may nucleate in liquid films, sheets or shells (see Section 4), and open spontaneously. The reason is that at the edge of a ruptured film, surface tension forces are no more balanced, and communicate inertia to the liquid. We discuss below the nature of this transfer, its sensitivity to the liquid properties, geometry (planar or circular), starting first by the integral formulation of Taylor $(1959 c)$.

A film of uniform thickness $h$ and surface tension $\sigma$ is bounded by a free straight edge with velocity $v=\dot{x}$. As the edge recedes under the action of capillary traction, 
it accumulates a mass $m$ initially constitutive of the undisturbed film, in a region of size $L$, which is the typical distance from the edge where fluid particles are accelerated irreversibly from $v=0$ (the film is at rest), to $v$. This region defines the extent of the sheet rim. The force, and mass balances (per unit rim length) over this region write

$$
\dot{P}=2 \sigma, \quad \text { and } \quad \dot{m}=\rho h v
$$

where $P=m v$ is the rim momentum. The factor 2 stands for the two sides of the film. Expanding the first relation in (3.13) as $m \dot{v}+v \dot{m}=2 \sigma$ we seek a constant receding velocity (as suggested by experiments, see Ranz (1959); Koros et al. (1960); McEntee \& Mysels (1969a); Frankel \& Mysels (1969); Pandit \& Davidson (1990)), leading to

$$
v=\sqrt{\frac{2 \sigma}{\rho h}}
$$

called the Taylor-Culick velocity. It was established along the above lines by Taylor $(1959 c)$ in the context of freely expanding liquid sheets and independently, in the circular geometry suitable to holes opening on a soap film, by Culick (1960), himself inspired by experiments of Ranz (1959). Equations (3.13) can be written

$$
\frac{d}{d t}\left(v \int_{0}^{t} \rho h v d t\right)=2 \sigma
$$

which, for a film with constant thickness indeed leads to $x \dot{x}=v^{2} t$, or $x=v t$ when $m(0)=0$. The velocity is constant, given by (3.14), from the start. Although the pulling force $2 \sigma$ is permanent, it is the rim velocity which is constant, and not its acceleration, because the mass $m$ is itself increasing on the way. The cases of an initial non-uniform film profile $h(x)$ was considered by Keller et al. (1995) and Raufaste et al. (2015), and non-zero mass by Lhuissier \& Villermaux (2011).

Culick offered a deeper understanding of (3.14) in correcting a notable, but interesting mistake by Dupré $(1867,1869)$, echoed in plain words by Lord Rayleigh (1891). The model of Dupré consisted in attributing the entire surface energy consumed when the rim has travelled a distance $x$ to the kinetic energy of the rim mass $m=\rho h x$, that is $\frac{1}{2} m v^{2} \neq 2 \sigma x$, a flawed equality leading to an over-estimated velocity $\sqrt{2} v$ when $v$ is given by the (correct) value in (3.14). The reason why this 'sentimental' energy balance (on which we come back in Section 11) fails is that it disregards the energy sink of the inelastic process of accelerating the fluid in the undisturbed film up to the velocity of the edge, a fraction of the available energy dissipated by internal viscous fluid friction which represents exactly half the consumed surface energy. Equivalently, this sink represents the energy loss at a sudden expansion when the fluid particles pass from the film to the thicker rim (see Section 11).

Estimating the viscous dissipation rate $\int \eta(\nabla \mathbf{v})^{2} d \Omega$ by $\eta(v / \delta)^{2} \Omega$ for a dissipation volume $\Omega \approx h \ell \delta$ and equating it to the rate of energy loss $\frac{1}{2}(2 \sigma \ell v)$ along a rim portion length $\ell$, Culick (1960) further computed the dissipation lengthscale $\delta$ as

$$
\begin{aligned}
\frac{\delta}{h} & \sim \frac{\eta v}{\sigma} \\
& \sim \frac{\eta}{\sqrt{\rho h \sigma}} \equiv O h,
\end{aligned}
$$

thus defining the Ohnesorge number $O h$.

The problem of a receding rim is thus one of the rare instances in Fluid Mechanics where the net amount of dissipation can be computed exactly from first principles, even in the presence of inertia. Like in turbulent flows, the dissipative scale self-adapts to make 
(i)
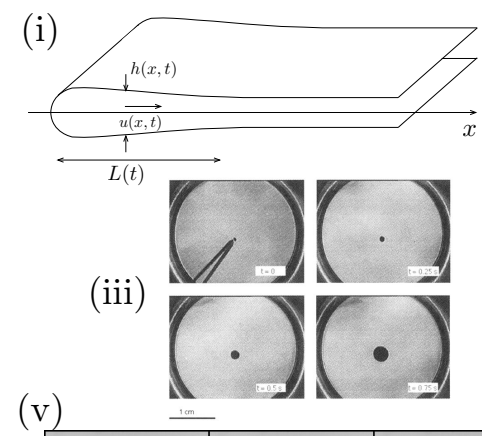

( v)

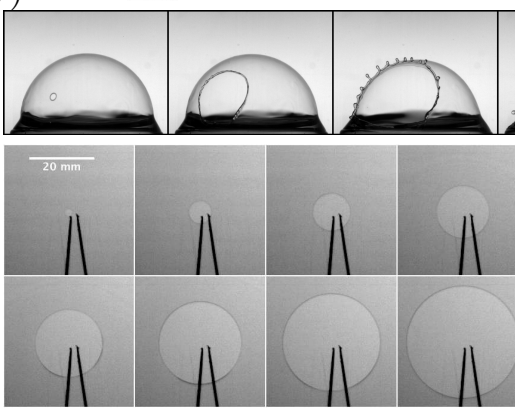

(iv) (ii)
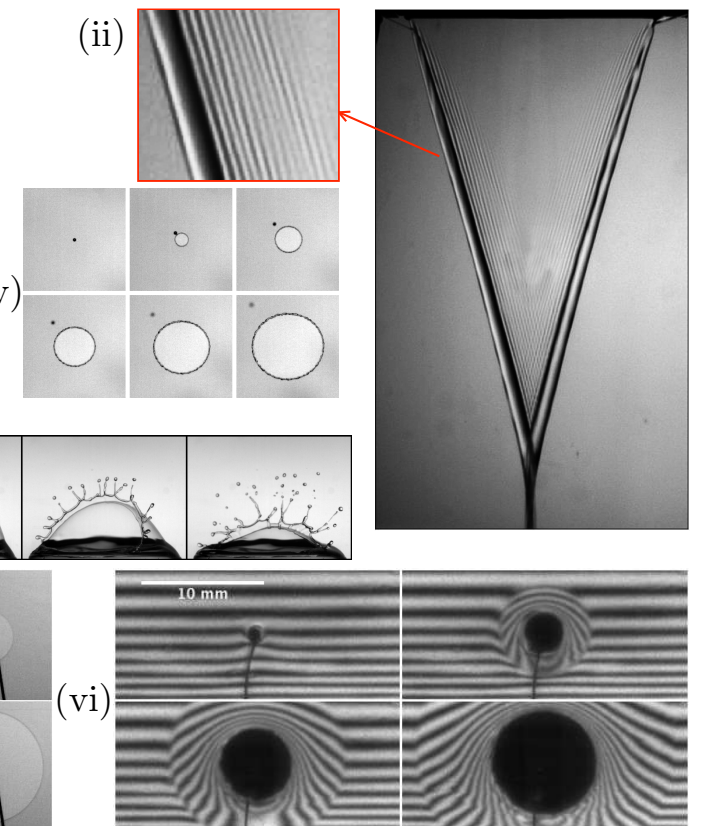

Figure 5. Bounded liquid sheets: rim recession and hole opening. (i) Sketch of a receding rim. (ii) A liquid sheet formed from a slit with edges receding at the constant Taylor-Culick velocity. Short capillary waves propagate ahead from the front. (iii) Hole expansion on a viscous film (Debrégeas et al. 1995). (iv) Hole expansion on a water film. (v) Hole expansion on a surface bubble (Lhuissier \& Villermaux 2012a). (vi) Hole expansion on a soap film seen in transparency (left) and in interferometry, highlighting the characteristic 'aureole' (Lhuissier \& Villermaux $2009 a)$.

the energy dissipation finite and independent of the fluid viscosity $\eta$ (thus including when $\eta \rightarrow 0$ ). It is, in this respect, the analogue of Taylor's microscale (Taylor (1935), or Liepmann-Taylor scale if one likes to view it as the thickness of internal shear layers (Dimotakis 2000)) in turbulent flows obtained by equating the viscous dissipation rate per unit mass $\nu(u / \delta)^{2}$ with the-viscosity independent- kinetic energy injection rate $u^{2} /(L / u)$ with $L$ and $u$ the stirring scale and velocity, respectively, leading to the familiar relation $\delta / L \sim R e^{-1 / 2}$ if $R e=u L / \nu$ is the Reynolds number based on the kinematic viscosity $\nu$ of the fluid. The end of the 'cascade' towards smaller scales where now $u(\delta) \sim u(\delta / L)^{1 / 3}$ is marked by the Kolmogorov scale $\delta / L \sim R e^{-3 / 4}$ (Kolmogorov 1941a). Like in the rim problem, $\delta \rightarrow 0$ when $\eta \rightarrow 0$ while the energy dissipation rate remains finite.

Viscosity does impact this problem however, through a not yet alluded to quantity, namely the rim extent $L$. We just saw that the dissipation scale $\delta$ in (3.17) adapts to fulfill the mass and momentum constraints in (3.13). The rim extent follows a qualitatively similar trend. For $O h \ll 1$, the liquid collects in a rim with a close-to-circular crosssection with radius $L \sim h\left[1+(v t / h)^{2}\right]^{1 / 4} \rightarrow \sqrt{v h t}$ given by mass conservation (see e.g. Gordillo et al. (2011)) and where recirculation motions dissipate the necessary amount of energy at a scale $\delta \ll L$.

For $O h \gg 1$ however, the picture is different. There is no collecting rim anymore, because $\delta$ is larger than $h$ from the start. Dissipation is spread-out diffusively along the - essentially flat- film which is now set into motion by longitudinal viscous stresses. Savva \& Bush (2009) have shown that in this limit, a fair, short time, slender slope $\left(h^{\prime} \ll 1\right)$ description of the velocity $u(x, t)$ along the film (with $\dot{x}_{0}=u\left(x_{0}, t\right)$ the film edge velocity) 
is given by

$$
\partial_{t} u=4 \nu \partial_{x}^{2} u+v^{2} \delta\left(x-x_{0}\right),
$$

valid for $x>h$. The film curvature $\kappa$ is of order $h^{-1}$ for distances to the edge of order $h$ and essentially zero elsewhere, so that its spatial variation at the edge location $x_{0}$ is caricatured as a Dirac Delta, that is $\partial_{x} \kappa \approx \frac{2}{h} \delta\left(x-x_{0}\right)$. The velocity along the film is thus given by the impulse response of a diffusion equation, providing

$$
\begin{aligned}
\dot{x}_{0} & \sim v \sqrt{\frac{t}{\tau_{\text {vis }}}}, \text { and } L \sim h\left(\frac{t}{\tau_{\text {vis }}}\right)^{3 / 2} \\
\text { where } \quad \tau_{\text {vis }} & =\frac{\eta h}{2 \sigma} \equiv \frac{\nu}{v^{2}}
\end{aligned}
$$

with $v$ the Taylor-Culick velocity in (3.14) above. The relaxation distance $L$ of the viscous stress from the film edge does depend on the liquid viscosity $\eta$, but this short-time behavior is a transient, and the film edge finally relaxes towards $v$, independent of $\eta$ (see also Chepushtanova \& Kliakhandler (2007)). During the transient as the edge accelerates, the global momentum balance $\dot{P}=2 \sigma$ in (3.13) is however always fulfilled, provided the film momentum $P=\rho \int h u d x$ is fully integrated, from the film edge in $x_{0}$ to distances far away from it, namely larger than $L$, which itself increases in time.

\subsubsection{Circular geometry: holes}

Liquid films and sheets may nucleate holes. We devote the entire Section 4.2 to the cause of the piercing event, but we describe here the opening dynamics of the hole once it has been formed. The circular geometry offers a slight complication in the sense that the border of the film curvature presents now two contributions, of opposite sign, reading $\kappa=1 / h-1 / r_{0}(t)$ where $r_{0}(t)$ is the hole radius. The classical study by Taylor \& Michael (1973) has elegantly shown that the shape of the hole envelope is analogous to the catenary discussed in Section 3.1.3 and that the condition for hole opening coincides with the condition for the catenoid stability: The hole has to be large enough $\left(r_{0} / h>0.76\right)$ to open spontaneously, otherwise it heals (i.e. $r_{0}(t) \rightarrow 0$ in a finite time, see Courbin \& Stone (2006) for an experimental illustration). The axisymmetric version of the balances in (3.13) is approximately given, in the inviscid limit for the scaled hole radius $\xi=r_{0}(t) / h$ with $t \equiv v t / h$, by

$$
\dot{\xi}^{2}+\frac{1}{2} \xi \ddot{\xi}=1-\frac{\pi}{4 \xi}
$$

which both restores the planar limit in (3.14), that is $\dot{\xi}=1$, and describes hole healing $\xi \sim(1-t)^{2 / 3}$ depending on $\xi$ being much larger or smaller than $\pi / 4 \approx 0.78$, respectively (see also Lv et al. (2018) for the healing dynamics in the presence of gravity).

The circular geometry has nevertheless a real consequence on this rim recession problem in the viscous limit. The analogue of the initial dynamics (3.18) describing now the radial velocity field $u(r, t)$ in the film for $r_{0} / h \gg 1$ is

$$
\partial_{t}(u r)=4 \nu\left(\partial_{r}^{2}(u r)-\frac{\partial_{r}(u r)}{r}\right)+v^{2} r_{0} \delta\left(r-r_{0}\right)
$$

a two-dimensional diffusion equation which solves for the hole radius velocity $\dot{r}_{0}(t)=$ $u\left(r_{0}, t\right)$ as

$$
r_{0}(t)=r_{0}(0) e^{t / 2 \tau_{\mathrm{vis}}},
$$

an exponential growth in contrast to the power law found in (3.19) for the planar limit (Savva \& Bush 2009). Geometry thus impacts the form of laws. 
Debrégeas et al. $(1995,1998)$ have given an "à la Dupré" description of this problem, by attributing the entire film surface energy consumed by the hole opening to the dissipation of energy by viscous stresses, an equality which fails quantitatively in the inertial limit as recalled above, but which is legitimate for $\mathrm{Oh} \gg 1$ when inertia is sub-dominant. Equating the rate of viscous dissipation

$$
\int_{r_{0}(t)}^{\infty} 2 \pi r h \frac{\eta}{2}\left[\left(\partial_{r} u\right)^{2}+(u / r)^{2}\right] d r
$$

with $u(r, t)=\dot{r}_{0} r_{0} / r$ to the rate of surface energy destruction $2 \pi \sigma h \dot{r}_{0}^{2}$ over an essentially constant in thickness film actually leads to (3.23).

Equation (3.23) is the axi-symmetric response to a genuine diffusive, viscous dynamics and should not, as noted by Debrégeas et al. (1995), be confused with a viscoelastic effect. When the mechanical response of the material is elastic, the initial stress propagation along the film is mediated by a shock wave, a phenomenon also observed in soap films coated by a rigid layer of surfactants (McEntee \& Mysels 1969b; Frankel \& Mysels 1969) and responsible for the formation of a so-called 'aureole' propagating upstream from the hole edge with a dramatic consequence on its stability (see Lhuissier \& Villermaux (2009a) and Section 6). When released, the extremity of a stretched rubber band recedes at constant velocity, proportional to the speed of sound in the material, and to a function of the initial stretch (Vermorel et al. 2007) while an isotropically stretched rubber sheet will, when ruptured at a point, more likely dissipate energy by opening radial fractures (Moulinet \& Adda-Bedia 2015).

\subsubsection{Savart sheet}

The premonitory intuition of Félix Savart was that by diverting promptly a liquid stream, one would gain information on the nature of the liquid itself. The configuration, declined in two memoirs (Savart 1833c,d) out of four (the two others being Savart $(1833 a, b)$, all published in 1833 in the Annales de Chimie Physique), consists in letting a jet impacting normally onto a small solid disk. It has indeed proved to be an extraordinary laboratory to study the consequence of liquid cohesion on the sensible world.

The incident momentum flux (per unity liquid density $\rho$ ) carried by the jet $\pi d_{0}^{2} / 4 u_{0}^{2}$ is cancelled by the reaction force of the impactor, and is distributed radially in an isotropic fashion. It is however reduced by an amount of order $\pi d_{i} \delta u_{0} u$, lost in the boundary layer at the surface of the impactor (with diameter $d_{i}$ ). The thickness of that boundary layer is of order (Schlichting 1987)

$$
\delta \sim \sqrt{\frac{\nu d_{i}}{u_{0}}} .
$$

If $\delta$ is usually small compared to the diameter of the impactor, it may compare with the sheet thickness itself whose inviscid value at the impactor lip is given by mass conservation

$$
h_{\mathrm{inv}}=\frac{d_{0}^{2}}{8 d_{i}}
$$

It is only when the Reynolds number $R e=u d_{0} / \nu$ is much larger than unity that the ratio

is appreciably small.

$$
\beta=\frac{\delta}{h_{\mathrm{inv}}}=2 \sqrt{\frac{\left(d_{i} / d_{0}\right)^{3}}{R e}}
$$

The step velocity across the sheet profile at the impactor lip relaxes by viscous smoothing as it leaves the impactor over a short radial distance of order $u_{0} h^{2} / \nu$ towards 
(i)

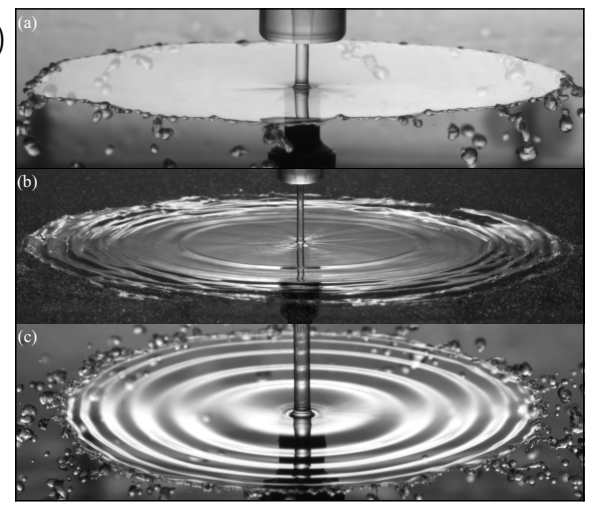

(iii)

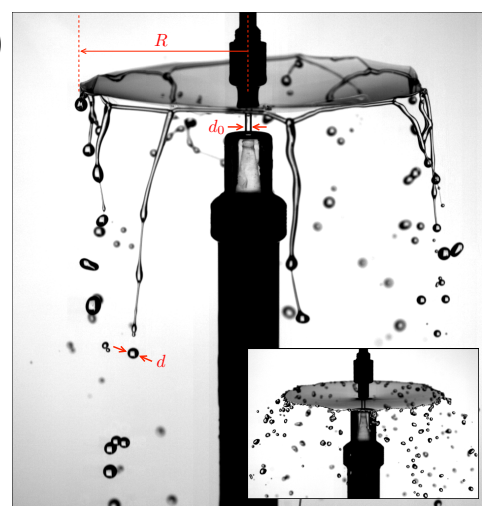

(ii)
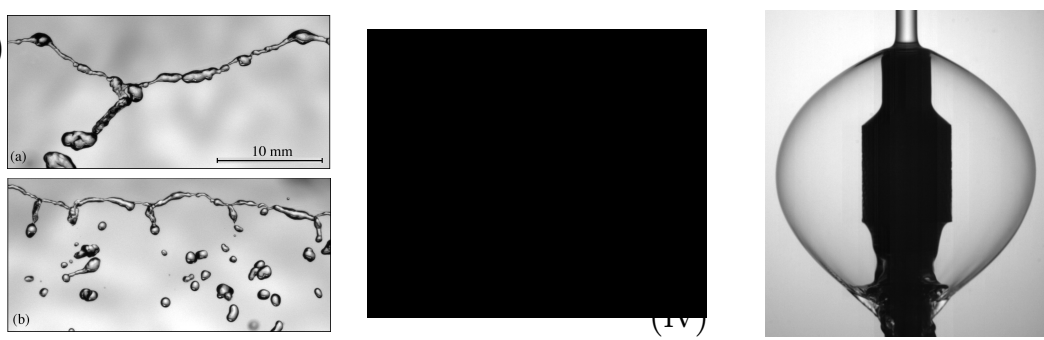

( $\mathrm{V})$

Figure 6. Savart sheets and bell. (i) Axisymmetric liquid sheet states: (a) Smooth regime. (b) Flapping regime for larger Weber number (higher than 1000). (c) Perturbation of the smooth regime by imposing vertical oscillations of the impact rod. (ii) Close-up views of the free rim of a smooth liquid sheet, for cases (a) and (b) above, adapted from Bremond et al. (2007). (iii) An instantaneous view of a viscous Savart sheet with a liquid 320 more viscous than water (the water case with the same injection parameters is shown in the insert, adapted from Villermaux et al. (2013)). (iv) Collision at an angle $\left(90^{\circ}\right)$ of two jets of ethanol (left) and water (right), adapted from (Bremond \& Villermaux 2006). (v) A stationary Savart bell exhibiting the characteristic catenoid shape.

a uniform (shear-less) profile with velocity $u$ and thickness $h$, carrying the rest of momentum $2 \pi r h u^{2}$, and the jet flow rate $\pi d_{0}^{2} / 4 u_{0}=2 \pi r h u$, thus providing (Villermaux et al. 2013)

$$
u=\frac{u_{0}}{1+\beta} \quad \text { and } \quad h(r)=\frac{d_{0}^{2}}{8 r}(1+\beta) .
$$

The ratio of the impulse carried by the sheet in a given radial direction (the average over the $2 \pi$ angular directions is zero for an axisymmetric sheet), to the incident impulse is thus

$$
\frac{\text { sheet impulsion }}{\text { incident impulsion }} \sim \frac{u^{2} h d_{i}}{u_{0}^{2} d_{0}^{2}}=\frac{1}{1+\beta},
$$

indeed smaller than unity. The above relations interpolate between the thin boundary layer limit $(\beta \ll 1)$ for which $u \approx u_{0}(1-\beta)$ and the viscous limit $(\beta \gg 1)$ which involves corrections in $\beta^{2}$ (Watson 1964).

Although the surface area of an element of volume travelling radially increases in proportion of $r / d_{0}$ (or of $d_{0} / h(r)$, equivalently), surface tension plays no role in its radial dynamics, which is purely ballistic. The reason is that the net force in the plane of the (essentially flat) sheet integrated over any closed contour

$$
\oint \sigma d \mathbf{s}=\mathbf{0}
$$


is exactly zero, as long as $\sigma$ is constant over the sheet, as noted by Marmottant et al. (2000). In the absence of other ingredients like the interaction with the surrounding ambient medium, or heterogeneous hole nucleation processes studied in the next sections, the velocity $u$ is thus purely radial, and conserved. Cohesion only compensates for the radial divergence of the streamlines in thinning the sheet for volume conservation, but at the expense of no net work (see in this respect the historical mistake of Hagen reported literally in Bouasse (1923), §107).

Following Taylor $(1959 c)$, it is admitted that the radial expansion is halted by capillary confinement when the Taylor-Culick velocity in (3.14) equals the radial speed $u$ or, equivalently, when the momentum flux of the sheet equilibrates surface tension forces, that is

$$
\rho u^{2} h\left(R_{\mathrm{TC}}\right)=2 \sigma .
$$

We will see in section 4 that this is not exactly true and that the corresponding sheet radius anticipated from $(3.31)$

$$
\begin{aligned}
& R_{\mathrm{TC}}=d_{0} \frac{W e}{16} \frac{1}{1+\beta} \\
& \underset{\beta \rightarrow 0}{\longrightarrow} d_{0} \frac{W e}{16} \quad \text { with } \quad W e=\frac{\rho u_{0}^{2} d_{0}}{\sigma}
\end{aligned}
$$

actually represents an upper bound. As $W e$ increases by an increase of the injection velocity $u_{0}$, the Reynolds number increases as well, and hence $\beta$ decreases. The overall dependence of $R_{\mathrm{TC}}$ on $W e$ is thus a steeper than the one known for the inviscid case $\left(R_{\mathrm{TC}} / d_{0} \sim W e\right)$, precisely because the relative momentum loss due to viscosity progressively fades away for larger $R e$. Also, viscosity delays the transition to the flapping régime to be analyzed in section 6 . There are two concomitant reasons for this delay: The liquid is slowed down, and the sheet is conversely thickened (3.28). For instance, the sheet thickness at the maximal smooth radial extension is

$$
h\left(R_{\mathrm{TC}}\right)=\frac{d_{0}}{W e}(1+\beta)^{2}
$$

It is thus doubly difficult for the weakened shear $u$ (by a factor $1+\beta$, see (3.28)) to move a heavier because thicker sheet (by a factor $(1+\beta)^{2}$ ) perpendicular to its plane and trigger an undulatory motion (even if the transit time has been augmented) hence the mandatory recourse to higher Weber numbers for it to be possible (see Section 6).

Note finally that the same concepts apply to non axi-symmetric impacts like those made from the collision of two identical jets at an angle, forming an elongated, bay leaf kind of shape which is well represented by elementary mass and momentum balances, as long as the rim does not fragment. The global extent of the sheet, its width and length, are both proportional to We, like the radius of a circular sheet (Hasson \& Peck 1964; Bremond \& Villermaux 2006).

\subsubsection{Savart bell}

Making a perfectly flat sheet from the impact of a perpendicular jet on a solid disk is actually difficult (see the method of Clanet \& Villermaux (2002) to achieve this singular limit). The angle $\alpha$ with which the sheet leaves the impactor may not be equal to $\pi / 2$. In that case, for instance when $\alpha<\pi / 2$, the sheet bends in an axi-symmetric way, forming a so-called bell, eventually closing at some downstream distance from the impact point (Savart 1833c; Boussinesq 1869a,b; Taylor 1959a). The reason for the bending is liquid cohesion, through Laplace pressure, balanced by the sheet centrifuge force. If $r(z)$ is the radius of the bell at the axial position $z$ from the impact point, the sheet presents two 
components of curvature, namely $R_{1}^{-1}=1 / r \sqrt{1+r^{\prime 2}}$ and the other $R_{2}^{-1}=r^{\prime \prime} /\left(1+r^{\prime 2}\right)^{3 / 2}$ in the plane containing the $z$-axis. When gravity is negligible and when the pressure difference between the inside and the outside of the bell is zero, equilibrium is described by

$$
\frac{\rho u^{2} h(r)}{2 \sigma} \frac{1}{R_{2}}=\frac{1}{R_{1}}+\frac{1}{R_{2}}
$$

where $u$ and $h(r)$ are both given in (3.28). Unlike for a bubble where the two radii of curvature are both equal to the bubble radius, the equilibrium above is not left unchanged by the substitution $R_{1} \leftrightarrow R_{2}$ (Clanet 2007). Symmetry is broken, and the bell is not spherical; it is actually a catenoid (Boussinesq $1869 a, b$ )

$$
\frac{L-r(z)}{L-r_{\mathrm{m}}}=\cosh \left(\frac{z-z_{\mathrm{m}}}{L-r_{\mathrm{m}}}\right)
$$

with $z_{\mathrm{m}}$ being the distance at which the maximal radius $r_{\mathrm{m}}$ is reached, both functions of the initial sheet deflection $r^{\prime}(0)=\tan \alpha$ and of the lengthscale $L=d_{0} W e / 16$ setting the overall size of the bell, which closes on the axis at $2 z_{\mathrm{m}}$. The influence of gravity, when aligned with the $z$-axis, can be quantified (Clanet 2007), and Lhuissier \& Villermaux $(2012 b)$ have studied the important case where the bell is pressurized with respect to its environment, leading to a singular, unstable shape studied in Section 5.2.

\section{Rupture of sheets and films}

Having considered how equilibria, static or dynamic, are ruled in some caricatural instances, we now come to breakup, starting with plane sheets and films.

Free liquid films -by free, we mean not confined between surfactants layers- are formed in various instances: they arise as a result of various jet impacts (see section 3.2.3) or splashes in the form of ejecta sheets (Worthington 1908), they constitute the shell bounding surface bubbles (Blanchard 1963), they can also be made on purpose through the extrusion of a liquid stream through a slit or annular thin jet for some spreading applications, in liquid propulsion devices, or ornament fountains (see Dombrowski \& Fraser (1954) and Fig. 1). Liquid films are of course also involved in various flows of effluents down inclined plates and coating processes (Craster \& Matar 2009; Kalliadasis et al. 2012).

Suspended liquid films, that is those not lying on a solid surface, usually breakup through their boundary. There, surface tension forces are unbalanced, pulling the liquid constitutive of the film into a receding rim, which undergoes various instabilities, before forming cusps, ligaments, and isolated drops.

\subsection{Phenomena at the rim: cusps}

The main shortcoming of Taylor's representation in (3.31) is that it is solely grounded on momentum equilibrium, disregarding the fate of the liquid accumulating in the rim. Another flaw is that in nature, the rims bordering liquid sheets do not remain straight for long, and soon present a series of indentations, particularly obvious on the steady state configuration of the Savart sheet.

In reality, the liquid does not accumulate in the rim, and is always removed in some way; there are plenty of mechanisms serving this purpose: by gravity dripping from stationary rims as those found in Savart sheets (Clanet \& Villermaux 2002; Villermaux et al. 2013), by centripetal forces when the liquid flows along a curved rim (Bush \& Hasha 2004; Bremond \& Villermaux 2006) or when the rim trajectory is curved like in 

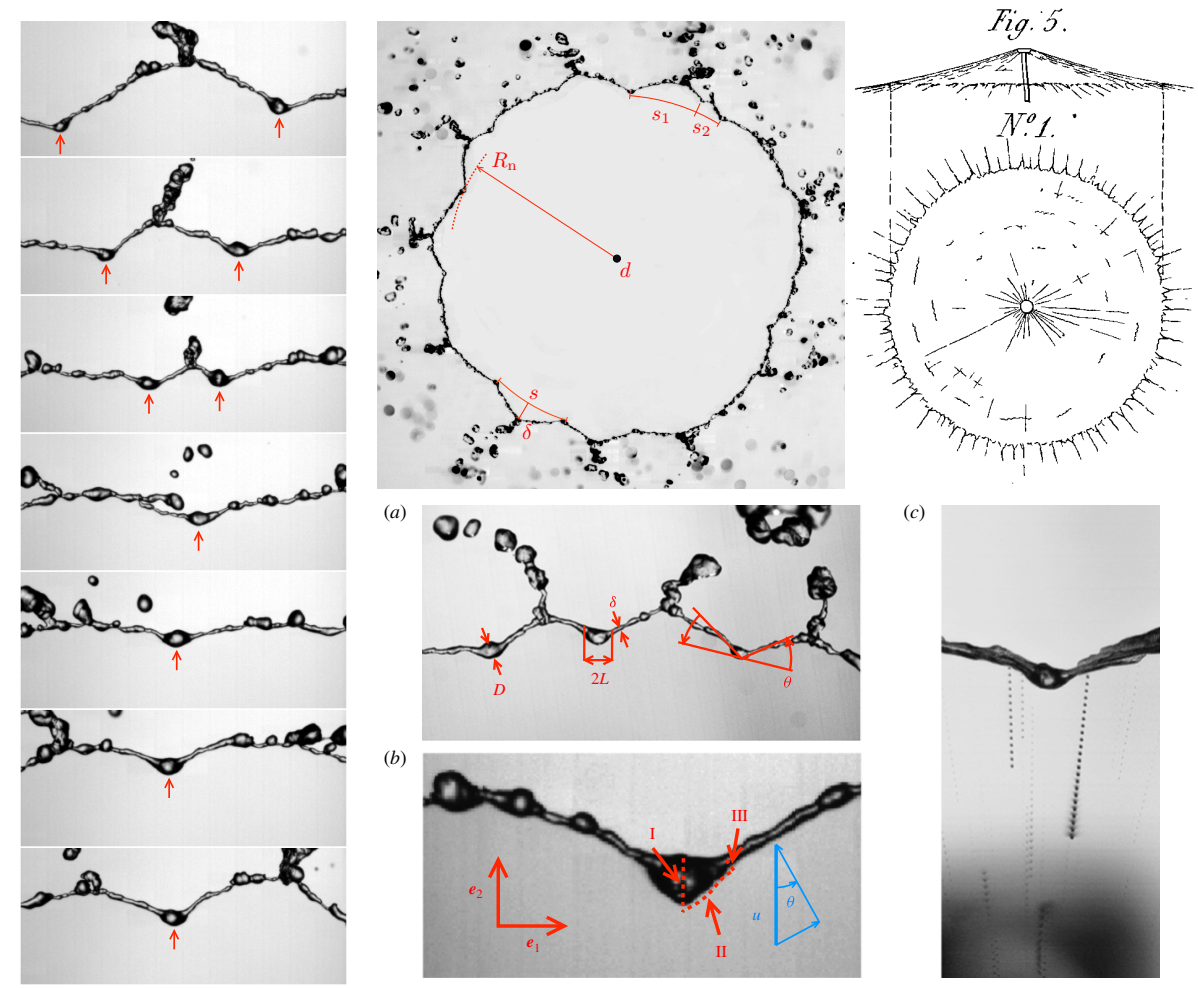

Figure 7. Cusps at the border of Savart sheets: shape and dynamics. Instantaneous views from above and a comparison with Savart's drawings, made by eye. There are in the mean of the order of $N \approx 10$ cusps around the sheet, at the tip of which droplets are formed. Their mean shape is well defined, but they also nucleate, coalesce as they move along the sheet (adapted from Gordillo et al. (2014) and Villermaux \& Almarcha (2016)).

spinning cups or bursting bubbles (Eisenklam 1964; Lhuissier \& Villermaux 2012a), by non-Gallilean body forces when the rim is decelerated (Villermaux \& Bossa 2011), by an electric field for charged liquids (Rozhkov et al. 2015; Brosseau \& Vlahovska 2017) etc... In all cases, the liquid is torn-off from the rim at a lengthscale prescribed by a corresponding instability, thus altering its straight shape, which gets distorted.

de Gennes (1996) had a very clever proposal to solve the Dupré-Rayleigh paradox recalled in Section 3.2.1, by offering a non-dissipative solution to the rim recession problem, involving droplets ejection from the rim, at $45^{\circ}$ out of the sheet plane. In that vision, both mass and momentum are conserved (as well as kinetic energy), with no mass accumulation by the rim. de Gennes was not truthfully convinced by his scenario which indeed proved to be incorrect (he writes: 'Up to now, as far as I know, nobody has seen droplets escaping during film rupture!' which is also incorrect, but for other reasons discussed in Section 6), a scenario which has nevertheless the right flavor of the true scenario.

The liquid is evacuated from the rim essentially in the sheet plane (modulo weak ejection orientation variations due to the turbulent motions in the rim) from singular structures called cusps by Gordillo et al. (2014). These were already visible from Savart's drawings (Fig. 7). Like in the de Gennes scenario, the cusp structure accommodates for both mass and momentum conservation although it leads, unlike in the de Gennes 
scenario, to a slightly shifted equilibrium condition compared to the Taylor equilibrium (3.33).

These cusps represent the saturated state of the rim instabilities caused by the various effects distorting the rim, like turbulent fluctuations and drop detachment for instance, and their robust geometry is constrained by both mass and momentum conservation principles. A sheet border is indented, each indentation presenting a bulge-like stagnation point at its base called node which drain the liquid through side jets towards the apex where these jets connect with the jets of their two adjacent cusps; there, the liquid is ejected in the form of a corrugated ligament, whose breakup produces the final drops.

If $h_{\mathrm{n}}$ is the thickness of the sheet, and $\theta$ the angle at the node of the cusp in the $\left(\mathbf{e}_{\mathbf{1}}, \mathbf{e}_{\mathbf{2}}\right)$ frame, Gordillo et al. (2014) have shown that the momentum exiting the stagnation bulge (of size $L$, proportional to $h_{\mathrm{n}}$ ) is

$$
\mathbf{m} \simeq \rho u^{2} h_{\mathrm{n}} L \mathbf{e}_{\mathbf{2}}-2 \sigma L \mathbf{e}_{\mathbf{2}}+\sigma L \mathbf{e}_{\mathbf{1}} .
$$

The rim orientation satisfies the condition for a stationary inclined shock, so that $\theta$ and $h_{\mathrm{n}}$ are linked by the condition that the momentum flux $\rho u^{2} h_{\mathrm{n}} \cos ^{2} \theta$ absorbed per unit length of the rim is balanced by the capillary forces $2 \sigma$ acting perpendicularly to it, and thus

$$
\cos ^{2} \theta=\frac{2 \sigma}{\rho u^{2} h_{\mathrm{n}}}, \quad \text { or } \quad \tan \theta=\sqrt{\frac{\rho u^{2} h_{\mathrm{n}}}{2 \sigma}-1} .
$$

Since the momentum flux exiting the node has the same direction as that of the rim, it follows from (4.1) and (4.2) that

$$
\begin{aligned}
\tan \theta & =\frac{\mathbf{m} \cdot \mathbf{e}_{\mathbf{2}}}{\mathbf{m} \cdot \mathbf{e}_{\mathbf{1}}} \\
& =2\left(\rho u^{2} h_{\mathrm{n}} / 2 \sigma-1\right)=2 \tan ^{2} \theta,
\end{aligned}
$$

giving the corner angle of the cusp at its node (see Fig. 7a)

$$
\tan \theta=\frac{1}{2}, \quad \text { that is } \theta \simeq 26.6^{\circ} \text {. }
$$

When applied to a Savart sheet where the thickness $h_{\mathrm{n}}$ and radius $R_{\mathrm{n}}$ at the node location are linked by (3.28), and further making use of relations (4.2) and (4.4), the radial position of the node is expressed as a function of the Taylor-Culick radius (with, say, $\beta=0$ as suitable for water), as

$$
\frac{1}{\cos ^{2} \theta}=\frac{\rho u_{0}^{2} d_{0}^{2}}{16 \sigma R_{\mathrm{n}}}=\frac{5}{4}, \quad \text { or } \quad R_{\mathrm{n}}=\frac{4}{5} R_{\mathrm{TC}} .
$$

The node radius is smaller than $R_{\mathrm{TC}}$ as is the radius $R_{\mathrm{e}}$ at which the cusps side jets collide, ejecting ligaments at velocity $u_{\mathrm{e}}$. Gordillo et al. (2014) have documented quantitatively the hierarchy

$$
R_{\mathrm{n}}<R_{\mathrm{e}}<R_{\mathrm{TC}}
$$

and have explained why, because the oriented side jets at the node carry momentum, thus cooperating with capillary retraction, equilibrium occurs for a sheet thickness $h_{\mathrm{n}}$ larger than the one expected by the naive balance (3.31). Because of this kick back to the rim, the 'effective' recession speed of an indented sheet is larger than the Taylor-Culick speed in (3.14), by a modest factor $\sqrt{5 / 4}$. Along the same lines, the ejection velocity of the ligaments is found to be approximately $u_{\mathrm{e}} \lesssim u_{0} \sin ^{2} \theta$, that is

$$
\frac{u_{\mathrm{e}}}{u_{0}} \lesssim 1-\frac{R_{\mathrm{n}}}{R_{\mathrm{TC}}}=\frac{1}{5}
$$


independent of $W e$, in relative agreement with the measurements reported by Taylor $(1959 c)$ whose ratio is closer to 0.15 , and those of Clanet \& Villermaux (2002) who report values ranging between 0.05 and 0.25 depending on $W e$. The ejection velocities are, moreover, distributed in intensity around the above estimate, and direction, scattered above and below the sheet plane. Section 11 considers the energetics of the process.

These cusps are not stationary. They are dynamic structures which evolve in time, are born, move, merge and die randomly along the sheet edge. However, their lifetime is much longer than the transit time of the liquid particles flowing through them for which they are frozen stationary structures, hence the description above. Their steady-state size distribution has been studied by Villermaux \& Almarcha (2016); elementary geometry shows that there are, in the mean $N \approx 10$ cusps at the periphery of a Savart sheet, so that the mean drop size from the breakup of the ejected ligaments is

$$
\langle d\rangle \sim d_{0} \sqrt{\frac{u_{0} / u_{\mathrm{e}}}{N}} \sim d_{0}
$$

unless they are stripped by gravity or centrifuge forces before reaching the cusps tips (Clanet \& Villermaux 2002).

We finally emphasize that these indentations are intrinsic to the dynamics of the sheet edge and are not the result of any extrinsic forcing. The fact that they have random and moving locations on the edge means that they do not result from some asymmetries in the jet or in the impact disk, unlike in the study of Taylor (1959c) and Dressaire et al. (2013), where the location and number of the cusps was forced by imposing large amplitude azimuthal modulations of the sheet thickness at the impactor, or in Lhuissier et al. (2014) where these modulations result from a viscoelastic instability in the impacting region.

\subsection{Spontaneous hole formation}

Liquid films also happen to puncture far from their boundaries, nucleating a hole, or a collection of adjacent holes on the plane of the film. We have seen in section 3.2.2 that holes need to be large enough in radius compared to the film thickness to open irreversibly (Taylor \& Michael 1973). The criterion is identical for polymeric membranes (Ilton et al. 2016) and is somewhat different for charged liquids which feature electrostatic repulsion at the hole lip (Betterton \& Brenner 1999).

While the sequence of phenomena occurring after a hole has been formed and opens in the plane of a film is well described and relatively well understood (see Section 4.1), the fundamental question of the hole nucleation process itself has been less explored. There are in fact many answers to this question, because there are many ways a film may puncture.

\subsubsection{Extremely thin films: thermal fluctuations}

Extremely small, thin objects are sensitive to thermal fluctuations. The minimal energy input to open a hole irreversibly is the surface energy $\sigma h^{2}$ (the hole radius must be of the order of the film thickness $h$, or more). That energy can, provided a scenario leading to film rupture exists, be supplied by the quantum $k_{B} T$ from the liquid thermal bath, leading

to $h \sim \sqrt{k_{B} T / \sigma} \approx 10^{-10} \mathrm{~m}$ in water at ambient temperature, a critical film thickness of the order of the inter-atomic distances. This thermally activated puncture scenario may thus at most apply to extremely thin films, like Newton black films (Casteletto et al. 2003). 
(i)

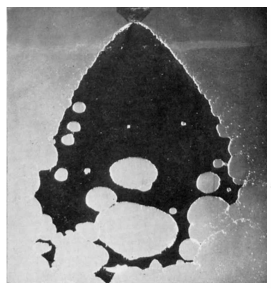

(iv)

(a)
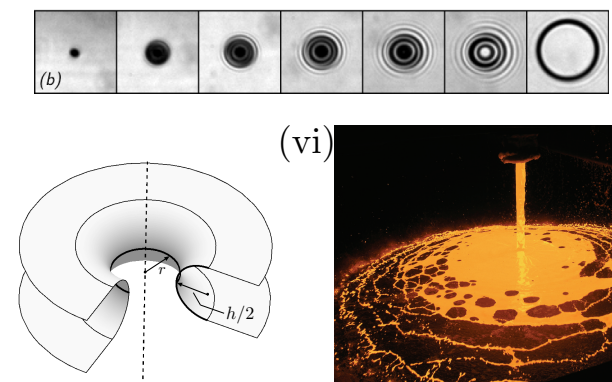

(vi)

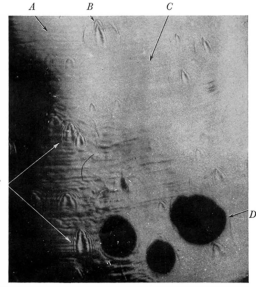

(ii)

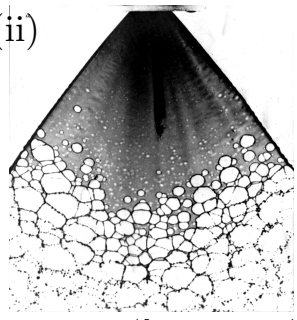

(iii)

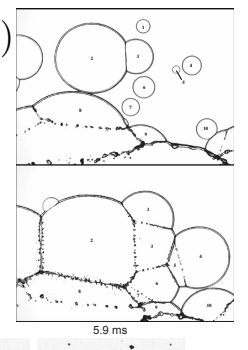

$5.9 \mathrm{~ms}$
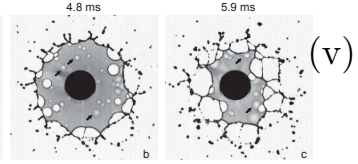

(vii)

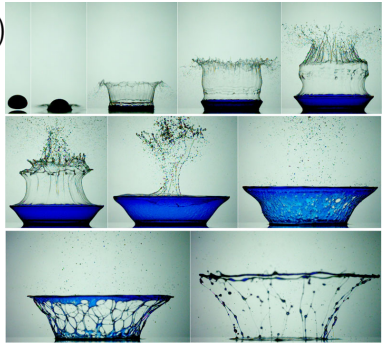

Figure 8. Nucleation of holes on thick (larger than a micron) liquid sheets. (i) Holes on a mercury sheet formed from flat fan atomizer, (ii) holes on a water sheet punctured by oil droplets dispersed in the liquid (adapted from Dombrowski \& Fraser (1954)). (iii) A network of holes punctured by bubbles in a water Savart sheet shown at two consecutive instant of time (Lhuissier \& Villermaux 2013). (iv) A water droplet coalesces in a water sheet (a) while an ethanol droplet punctures it (Néel \& Villermaux 2018). (v) Impact of a drop loaded with partially miscible oil droplets, nucleating many holes as it expands (adapted from Vernay et al. (2015a)). (vi) Disintegration of a liquid iron jet poured onto a refractory brick (Lhuissier \& Villermaux 2013). (vii) Impact of a glycerine drop onto a thin substrate film of isopropanol. The ejecta sheet punctures in a myriad of holes (Aljedaani et al. 2018).

\subsubsection{Very thin films: van der Waals forces}

Intermolecular forces have different origins depending on whether the molecules are charged, permanently dipolar, or dipolar by induction. These forces, called generically van der Waals forces (Israelachvili 1991), are attractive over a range of distances which compares to the size of the molecules itself, namely a few nanometers. The corresponding disjoining pressure $-A / h^{3}$ where $A$ is a Hamaker constant (Derjaguin et al. 1987), is responsible for puncturing very thin films up to a few tens of nanometers (Vrij 1966; Reiter 1992; Sharma \& Reiter 1996; Erneux \& Davis 1993; Champougny et al. 2017), but does not operate with much thicker films, despite the fact that appreciably thicker films, of the order of $200 \mathrm{~nm}$ (Thoroddsen et al. 2012b), and even more (Nierstrasz \& Frens 1998) have been claimed to be sensitive to this effect.

\subsubsection{External solicitations}

A liquid film can be transpierced by a sharp (with a radius of curvature smaller than the film thickness) solid object, a projectile (see Fig. 5 and Bull (1904); Courbin \& Stone (2006)), a concentrated air jet (Berendsen et al. 2012; Lhuissier et al. 2016), a focused laser beam (Wedershoven et al. 2015) or a spark (McEntee \& Mysels 1969a; Lhuissier \& Villermaux 2009b), whose action is applied for a sufficiently long time. Like all fragile objects, liquid films are also sensitive to the unsteadiness of their environment. The application of a pressure gradient, or pressure difference across a film sets it in motion, 
and the corresponding acceleration may, because the film is a density interface when surrounded by a gas, lead to destabilization through a Rayleigh-Taylor mechanism. An impulsive pressure wave (Bremond \& Villermaux 2005), or a violent explosion (Vledouts et al. 2016a) cause the film, accelerated perpendicular to its plane, to grow thickness modulations which ultimately cause its puncture with a well prescribed wavelength examined in section 6 . The same phenomenon is responsible for the crumpling of liquid bells, and the formation of transverse indentations at the edge of flapping liquid sheets (Lhuissier \& Villermaux 2012b); it may affect films of arbitrary thicknesses, the studies just mentioned had microns, to tens of microns thick films.

\subsubsection{Internal flaws and defects}

Solid hydrophobic particles introduced into a film can, when their size compares with the film thickness, lead to its rupture as they force the two interfaces of the film to pinch at the surface of the particle. Anti-foaming agents have taken advantage of this effect for a long time (Garrett 1992; de Gennes 1998). However flaws, impurities or defects in the liquid may not be solid. Immiscible oil droplets (Dombrowski \& Fraser 1954), or bubbles (Lhuissier \& Villermaux 2013) also act as efficient hole nucleation sites in water films for precise reasons that remain largely elusive. Denkov (2004) notes that, when by chance an oil droplet reaches an aqueous interface, the resulting surface tension contrast (oils have usually a weaker surface tension than water) drives a well-defined outward superficial flow. Vernay et al. (2015a), adapting this result to a radially expanding liquid sheet loaded with partially miscible oil droplets argue that it is responsible for the film piercing, which they do observe experimentally.

\subsubsection{Surface inhomogeneities and Marangoni stresses}

Chemical and temperature inhomogeneities at the surface of a liquid translate into inhomogeneities of surface tension. The corresponding Marangoni surface stress (Marangoni 1878 ) is communicated to the bulk of the liquid by viscosity in a way, when the liquid is shallow like in films, which can be dramatic (Scriven \& Sternling 1960). In the Bénard problem of a liquid layer heated from below for instance (Bénard 1900, 1901), the mean temperature decays from the hot plate to the liquid free surface. Since surface tension of a liquid typically decreases with temperature, this gradient yields a surface shear stress when the interface is distorted, setting the liquid into motion towards the cooler areas, where the surface tension is higher (Levich \& Krylov 1969). There, the film thickens when at the same time, the flow away from the hotter regions results in film thinning. Any initial disturbance of the film thickness or of the interface temperature is thus amplified (Pearson 1958). This instability may lead to film rupture, and local drying of the heating plate (Kabova et al. 2006; Vanhook et al. 1997; Boos \& Thess 1999), namely the analogue of holes formation. This effect, which may also be due to differential evaporation in mixtures (Guéna et al. 2007) is, for this reason, used as an efficient cleaning process (Leenaars et al. 1990; Matar \& Craster 2001). Other experiments of Marangoni stress induced spreading flows include those of Roché et al. (2014) and Hernández-Sánchez et al. (2015) suggesting that this kind of mechanisms can affect films up to a millimeter thick; when coupled to evaporation, it may also provoke the spontaneous emulsification of weakly miscible mixtures (Keiser et al. 2017; Wodlei et al. 2018).

\subsubsection{Thick, spontaneously puncturing films}

Yet, if the case of extremely, or very thin films (Sections 4.2.1 and 4.2.2) is satisfactorily understood, the case of thick films being neither sensitive to thermal fluctuations, nor to a disjoining pressure, in the absence of sustained temperature, or pollutant concentration 
gradient (Section 4.2.5), remains an enigma. Even the precise role of defects (Section 4.2.4), when they are temperature or soluble chemical agent spots localized in space in, or at the surface of the film, needs to be clarified. A fundamental issue is to know whether a localized surface inhomogeneity spontaneously leads to film rupture in a finite time (Bowen \& Tilley 2013), or not (Kitavtsev et al. 2018), besides the possible role of surfactants (Jensen \& Grotberg 1992, 1993). The problem is particularly acute for liquid films with high surface tension like water (Lhuissier \& Villermaux 2012a) or mercury (Dombrowski \& Fraser 1954) whose surface is easily contaminated by ambient pollutants, those films being known to puncture spontaneously even if paradoxically thick in the sense of the above classification (Aljedaani et al. 2018).

Néel \& Villermaux (2018) have uncovered a mechanism solving the paradox, relying on the extreme sensitivity of the film to surface tension inhomogeneities. The surface tension of a free liquid film is lowered by an amount $\Delta \sigma$ over a size $a$ by chemical or thermal contamination. At the same time this spot diffuses (within a time $a^{2} / D$, with $D$ the diffusion coefficient of the pollutant in the substrate), the Marangoni stress $\Delta \sigma / a$ induces an inhomogeneous outward interstitial flow which digs the film within a time

$$
\tau_{0} \sim \sqrt{\frac{\rho h a^{2}}{\Delta \sigma}}
$$

with $\rho$ the density of the liquid. When the Péclet number

$$
P e=\frac{a^{2}}{D \tau_{0}}
$$

is larger than unity, the liquid substrate motion reinforces the surface tension gradient, triggering a self-sustained instability insensitive to diffusional regularization. The paradigm is that liquid surfaces are always somewhat dirty to some extent (Poulain et al. 2018), and that even a minute amount of dirt is enough to initiate puncture. The criterion in (4.9)-(4.10) makes precise what 'dirt' means.

\subsection{Kinematic thinning}

By contrast with a round jet, a flat liquid sheet initially at rest, or animated with a uniform translational velocity along its plane will not amplify thickness corrugations spontaneously, because surface tension is stabilizing for a flat sheet (see Section 8 and Appendix A.5). However, a liquid sheet may be prepared in such a way that the velocity $u(x, t)$ of the fluid particles averaged over its thickness $h(x, t)$ is initially inhomogeneous along $x$, namely when $u(x, t=0)$ is not a constant. Analogously, the velocity at the origin of space $u(0, t)$ may be fluctuating in time, as it is notoriously the case for flat fan injectors used in the agricultural spraying context for which the liquid is turbulent at the nozzle exit. In that instance, the sheet will locally thin or thicken depending on the sign of the initial velocity gradient $(\partial u / \partial x)_{t=0}$ when spatial modulations of $u(x, 0)$ are imposed to the sheet, or of the sign of the acceleration $(\partial u / \partial t)_{x=0}$ when the velocity $u(0, t)$ is modulated in time at the injector exit. This results from trivial kinematics: if a slow particle released in $x=0$ is preceded by a fast particle, their separation distance will further increase, thus thinning the sheet in-between them. Conversely, a fast particle will catch-up a slow particle ahead to form a shock. This phenomenology applies to jets 
(i)

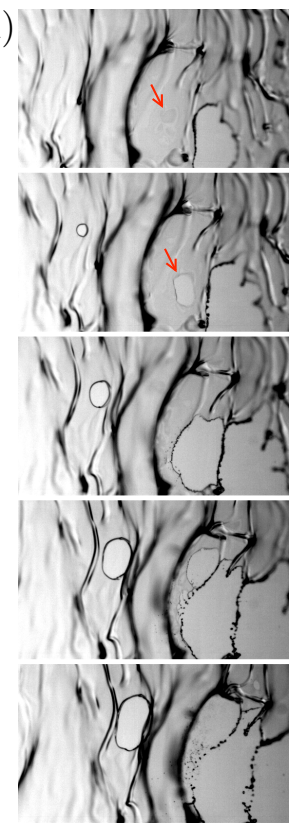

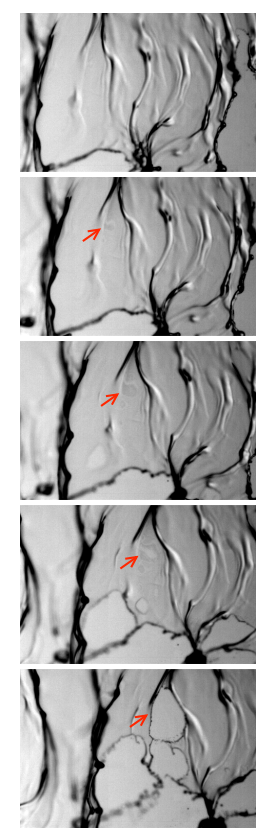
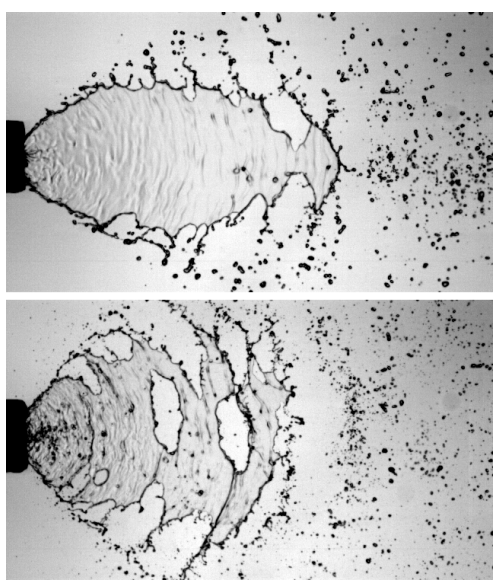

(ii)
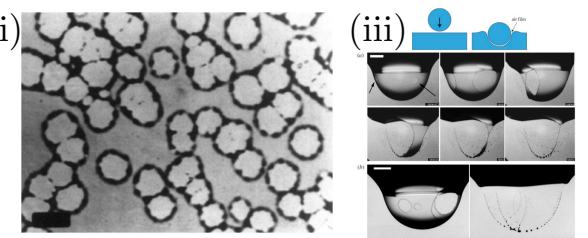

Figure 9. Kinematic thinning leads to film rupture. (i) Agricultural flat fan nozzles (Albuz) produce a turbulent sheet, possibly loaded with bubbles. Initial injection velocity modulations lead to thinning of the sheet along large valleys which, when they are thin enough (of the order of $1 \mu \mathrm{m}$ or less), nucleate holes spontaneously. The arrows indicate the regions where this scenario occurs. This case has to be contrasted with hole nucleation on very thin sheets where van der Waals forces are at play: (ii) 40 nanometer thick polymer film on a solid substrate (Reiter 1992) and (iii) bursting of relatively thin (250 nanometer) air films (Thoroddsen et al. 2012b).

also (Meier et al. 1992). The sheet thickness $h(t)$ in Lagrangian time $t$ reads

$$
\begin{aligned}
\frac{h(0)}{h(t)} & =1-t \times\left.\frac{1}{u} \frac{\partial u}{\partial t}\right|_{x=t=0} \\
t & =\frac{x}{u(0,0)} .
\end{aligned}
$$

It blows-up in finite time at concentrated shocks when $(\partial u / \partial t)_{x=0}>0$, and decays like $t^{-1}$ around large valleys when $(\partial u / \partial t)_{x=0}<0$, as seen in Fig. 9 which also shows how the thin, stretched sheet in the valleys nucleate holes spontaneously, holes which expand preferentially along the valleys, where the liquid film is the thinnest. There, the sheet thickness, computed from Taylor-Culick velocity in (3.14) is found to be about $1 \mu \mathrm{m}$, a value often encountered with clean water which coincides, at least quantitatively, with its Debye screening length (Israelachvili 1991), although no precise connection has been made yet (see the different scenarii discussed in Yaminsky et al. (2010)).

Of course, surface tension comes into play in the strongly distorted regions of the film interface, namely around the neck between valleys and shocks. Burton \& Taborek (2007) have shown that the inviscid, slender slope conservation equations

$$
\begin{aligned}
\partial_{t} u+u \partial_{x} u & =\frac{\sigma}{\rho} \partial_{x}^{3} h \\
\partial_{t} h+\partial_{x}(h u) & =0
\end{aligned}
$$


are compatible with a finite-time singularity $h(t) \sim\left(t_{\star}-t\right)^{\alpha}$ with $\alpha \approx 0.7$, presumably due to a Bernoulli suction effect at the neck.

\section{4. 'Effervescent' Atomization}

The terminology 'effervescent', or 'aerated liquid' atomization and the corresponding technique was introduced by A. H. Lefebvre in the late eighties (see Sovani et al. (2001) for a review). It consists in dispersing a minute fraction of a gas phase within the liquid to be atomized when the mixture is pressurized prior to its injection in a low pressure chamber. As the pressure suddenly drops, the gas bubbles expand and fragment the liquid into small volumes, typically much smaller than those which would have been obtained if the same quantity of gas had been used to shear the liquid, as in common co-flow atomizers (Lefebvre 1989), like coaxial jets (Marmottant \& Villermaux 2004b). This 'effervescent' process, though not routinely used yet is thus a smart alternative for which a small cause (the addition of a small quantity of gas), has dramatic effects (substantially reduced drop sizes), even under modest driving pressures. Its efficiency relies on one hand in the change of topology of the liquid in the injection stream resulting from the presence of the bubbles, and on the other hand in the fast bubble expansion dynamics as the pressure suddenly releases. The coupling between these two effects, and the associated detailed mechanisms were investigated by Lhuissier \& Villermaux (2013) using a Savart sheet seeded with small air bubbles, themselves inspired by the work of Dombrowski \& Fraser (1954) who were using a fan spray nozzle and a water/oil emulsion (see Figs 8 and 9).

Note that the presence of a dispersed phase within the liquid to atomize is not mandatory for the realization of the first step listed above, that is the nucleation of holes. A similar effect is obtained by using a mixture of surfactants inducing Marangoni stresses, which eventually puncture the sheet (see Rozhkov et al. (2010); Vernay et al. (2015a) in the context of drop impact). This mechanism is also similar to the spontaneous de-wetting (through hole nucleation, or spinodal decomposition) of a thin film from a solid surface (Reiter 1992) even if it leads to a very different drop sizes population. The impact of fine droplets of another liquid mediates the nucleation of holes as a result of Marangoni localized stresses (Thoroddsen et al. 2006; Aljedaani et al. 2018). Hole formation on a liquid film may also be the result of an inertial instability, as when the film is violently accelerated perpendicular to its plane (see Sections 5 and 7 ), leading to patterns very like those in Bremond \& Villermaux (2005). In the industrial practice, Fig. 8 illustrates that the two former steps leading to the spray are for instance observed in the GranShot process (Uddeholm AB Sweden) where a single-phase iron melt is fragmented through the disintegration of a planar liquid sheet. This process is a major industrial method for granulation of melts at large output, up to several tons per minute. It is however reputed to be "... not very flexible in term of particle size and shape, these being largely determined by the physical and chemical characteristics of the melt" according to Yule \& Dunkley (1994) p. 216, and one could therefore expect 'effervescence' to give more flexibility, if not control, to the process.

Overall, whatever the precise hole piercing mechanism may be, the process features the following successive steps:

(i) Nucleation of holes across the sheet, in a more or less synchronous way,

(ii) Growth of these holes followed by the junction of the rims bordering them into a web of ligaments which concentrates all the liquid constitutive of the sheet,

(iii) Break-up of this ligaments web into the collection of drops.

The key quantity is the surface density of the holes, or inter-holes centers distance $\lambda$. It is easy to see that, by volume conservation, the average diameter $\langle d\rangle$ of the ligaments 

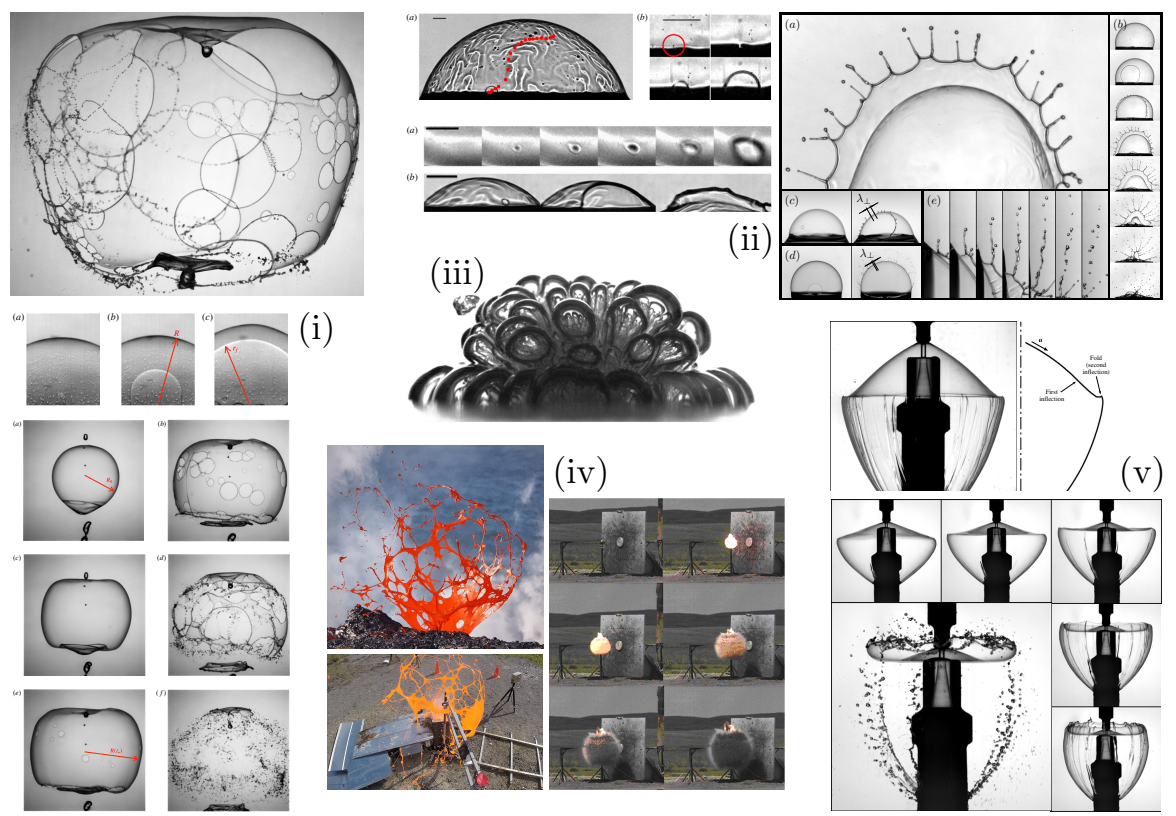

FIgURE 10. Rupture of liquid shells. (i) Explosive fragmentation of a liquid shell expanded by an exothermic chemical reaction (Vledouts et al. 2016a). (ii) Nucleation of a hole a the foot of a water bubble at the surface of a pool, and its fragmentation (adapted from Lhuissier \& Villermaux (2012a) and Poulain et al. (2018)). (iii) Explosive boiling (van Limbeek et al. 2013). (iv) Explosion of magma bubbles recorded in nature (Gonnermann 2005) and re-created artificially (Sonder et al. 2018). (iv) Explosion of a cohesive ball of metal spheres (Frost et al. 2007). (v) Fragmentation of a crumpled water bell (Lhuissier \& Villermaux 2012b).

formed from a sheet with thickness $h$ is

$$
\langle d\rangle \sim \sqrt{\langle\lambda\rangle h}
$$

a diameter which also sets the mean drop size, as will be seen in Section 8. Lhuissier \& Villermaux (2013) have further shown that the overall drop size distribution from this 'effervescent' process is universal provided the holes are not too distant (Néel et al. (2020) have quantified what 'distant' means and how the drop size distribution is correspondingly altered, see Section 10), and that it is solely related to the breakup dynamics of the ligaments, irrespective of the geometric disorder of the holes at the surface of the sheet (i.e. of the distribution of $\lambda$ ). This drop size distribution will be discussed in Section 9.

\section{Shells}

Liquid sheets are often bent by a pressure difference between their sides, like for inflated bubbles for instance. A spherical liquid shell, or a bubble lying at the surface of a pool are not bordered by a rim. Their bursting mechanisms are therefore different from those of bounded liquid sheets.

\subsection{Exploding bubbles and drops}

We consider envelopes of cohesive material enclosing a substance or a mixture of substances whose internal pressure increases rapidly, in other words, an explosion. This 
configuration is the three dimensional analogue with liquids of the ring configuration initially imagined by Mott (1947) for solids in two dimensions (see also Grady (2006); Zhang \& Ravi-Chandar (2007, 2008), and Section 2.3 for its discrete version with magnets). This problem, in which the envelope fragments distribution is the result of a competition between deformation and cohesion, is relevant to a collection of phenomena spanning over a broad range of length scales, among which are: Exploding blood cells and bacteria (antibiotics like penicillin disrupt cell walls by explosive lysis, Flores-Kim et al. (2019)), spore dispersal from plants (Ingold 1971; Hassett et al. 2013), boiling droplets (Frost 1988; van Limbeek et al. 2013; Antonov et al. 2019), underwater explosions (Cole 1948), magma eruption in volcanoes (Kedrinskii 2009; Sonder et al. 2018), up to the torn patterns of supernovae in the Universe (Burrows 2000), among other examples. Case shells, bombs are obvious examples where one would like an a-priori knowledge of the final fragments as a function of the energy released by the explosion, and of the physical properties of the enclosing envelope (Zeldovich \& Raizer 2002; Kedrinskii 2005; Frost et al. 2007).

The problem examined here is best discussed in the spherical geometry, and admits two limits, from the strong, supersonic version involving a detonation (Sedov 1946), a 'very intense explosion' in the words of Taylor (1950a), to the milder deflagration version consisting, in expanding a liquid bubble filled with a gas mixture undergoing an exothermic reaction, like a hydrogen/oxygen mixture for instance (Marangoni \& Stefanelli 1873), which propagates at low Mach number within the bubble. The interest of this configuration is that the course of events can be followed step by step and in real time, from an initially connex cohesive material (here a liquid shell), to its dispersion into a collection of stable fragments, each step being subjected to a precise description (Vledouts et al. 2016a).

\subsubsection{Explosive fragmentation of a liquid shell}

A reactive mixture is ignited inside a liquid bubble. A flame propagates at constant velocity $\dot{r}_{f}$, converting the fresh mixture with density $\rho_{u}$ into burnt gases with density $\rho_{b}$. Since the burnt gases are much lighter than the fresh mixture and are released on the inner confined side of the flame, they must expand, and by doing so, push the different phases ahead of the flame front. These are, successively at increasing distances from the flame, the envelope of the remaining fresh gases, the liquid shell, and the ambient air. The radial motion of these inertial phases is thus made at the expense of an elevation of pressure in the burnt gases with respect to the initial ambient pressure. The important quantity is the shell radius acceleration $\ddot{R}$. Vledouts et al. (2016a) have shown that it is related to the pressure at the flame front by

$$
p\left(r_{f}\right)=\rho_{u} \ddot{R}\left(\frac{R^{2}}{r_{f}}+\frac{\rho}{\rho_{u}} h+\frac{\rho_{\infty}}{\rho_{u}} R\right)
$$

where $\rho$ is the density of the liquid shell, $h$ its thickness, and $\rho_{\infty}$ the density of the outside medium (air). The pressure at the flame front is of the order of the recoil pressure $\rho_{u} \dot{r}_{f}^{2}$ and since the first term dominates in the right hand side of (5.1) for short times after ignition when $r_{f}=\dot{r}_{f} t \ll R$, the shell radius acceleration is such that

$$
\rho_{u} \frac{R_{0}^{2} \ddot{R}}{r_{f}}=\frac{3}{2} \rho_{u} \dot{r}_{f}^{2},
$$


where $R_{0}$ is its initial radius, giving

$$
\frac{R}{R_{0}}=1+\frac{1}{4}\left(\frac{r_{f}}{R_{0}}\right)^{3}
$$

The shell radius expands like $R(t) \sim t^{3}$, with an acceleration $\ddot{R} \sim t$ itself strengthening in time, a violent explosion indeed.

\subsubsection{Stability}

The dense spherical shell is sandwiched between two media with lighter densities (the inner unburnt gases, and the outer ambient air), so that, being accelerated radially, it is liable to develop an inertial instability in the sense of Rayleigh-Taylor (see Appendix A.1 and A.2). The development of this instability is, however, altered by the presence of two close-by density interfaces, a proximity which is stabilizing (Keller \& Kolodner 1954), as first demonstrated experimentally by Bremond \& Villermaux (2005). If $\ddot{R}$ is the acceleration of a density interface, the cut-off wavenumber of the instability is

$$
k_{c}=\sqrt{\frac{\rho \ddot{R}}{\sigma}}
$$

whose inverse must be compared to the shell thickness $h$. If $k_{c} h \gg 1$, the standard analysis of Lord Rayleigh (1883) holds, for which both the most amplified wavenumber $k_{c} / \sqrt{3}$, and its growth rate $\sim\left(\rho \ddot{R}^{3} / \sigma\right)^{1 / 4}$ are independent of $h$. But when

$$
k_{c} h \ll 1
$$

the coupled distortions of the nearby interfaces hinders the instability development, which now occurs around a wavenumber given by (see Appendix A.2)

$$
k \approx \frac{1}{2} k_{c}^{2} h, \quad \text { with a growth rate } \omega=\sqrt{\frac{\rho \ddot{R}^{2} h}{2 \sigma}}
$$

going to zero as $h \rightarrow 0$. The rear and front (in the direction of the shell expansion) interfaces amplify disturbances inducing thickness modulations of the shell, piercing it when they are of the order of its initial mean thickness.

Using the expression for the growth rate in (5.6), and further defining the relevant Weber number

$$
W e=\frac{\rho \dot{r}_{f}^{2} h}{\sigma},
$$

we find the instability time $t_{\star}$ and destabilization radius $R\left(t_{\star}\right)$

$$
\frac{\dot{r}_{f} t_{\star}}{R_{0}} \sim W e^{-1 / 4}, \quad \text { and } \quad \frac{R\left(t_{\star}\right)}{R_{0}}-1 \sim W e^{-3 / 4}
$$

It follows that the instability wavelength $\lambda$ at the piercing time is (Vledouts et al. 2016a)

$$
\frac{\lambda}{R_{0}} \approx \frac{2 \pi}{W e^{3 / 4}}
$$

defining the inter-distance between the growing holes which, through the 'effervescent' mechanism described in Section 4.4, also sets the mean drop size as in (4.15). The shape of the drop size distributions is discussed in Sections 9 and 10. 


\subsection{Singular Savart Bell}

A liquid film flowing along a curved path experiences centripetal acceleration, as in Savart bells, a particular liquid shell resulting from the dynamical equilibrium described in Section 3.2.4.

Stationary axi-symmetrical pressurized bells (i.e. with internal pressure larger than the ambient) can exhibit sharp pointed shapes. They are characterized by a double inflection of the bell generator profile (with curvature $R_{2}^{-1}$ ), corresponding to not globally convex bells. Lhuissier \& Villermaux (2012b) have shown that this shape is incompatible with the usual assumption that the detail of the flow across the liquid sheet constitutive of the bell is unimportant. They have considered the equilibrium of a curved liquid sheet of finite thickness, that is when

$$
\frac{h}{R_{2}}
$$

is small, but nonzero, sustaining a pressure difference between both sides, and have shown that several curvatures may be a solution under given flow conditions. The inflection of the bell profiles is then explained in terms of a spontaneous transition from a 'negative' to a 'positive' curvature which conserves mass flow, linear and angular momenta. That inflection is also a transition from a super to a subcritical flow (with respect to capillary waves with velocity given by (3.14)), having the status of a capillary hydraulic jump on a freely suspended sheet, a novel object in Fluid Mechanics.

The local curvature of the sheet along a streamline can be very large at the critical condition. This is the cause for the azimuthal wrinkles forming at the jump which result from the inertial destabilization of the sheet (a finite-thickness Rayleigh-Taylor instability as described above and in Appendix A.2), due to the centripetal acceleration fluid particles experience as they flow along the highly curved bell profile in the vicinity of the singular sheet fold. This finding, explaining why the bells are so fragile in these conditions and rupture easily, also explains the singular shape at the edge of freely flapping sheets, discussed in Section 6.

\subsection{Surface bubbles}

A surface bubble is an out-of-equilibrium, close-to-hemispherical liquid shell sitting at the surface of a liquid pool in which it progressively empties. Its shape depends on both gravity and capillary while its cap continuously drains down to rupture. It is one of the most common objects in nature since an immensely large number of bubbles burst every second at the surface of the ocean, feeding the atmosphere in a considerable amount of water vapor, salt and a collection of chemical/biological material (Veron 2015; Cochran 2017).

A bubble bursts because its cap (the liquid shell) spontaneously nucleates a hole on its surface, preferentially close to its foot. At burst, two distinct processes (discovered by Knelman et al. (1954)) further produce fragments, the 'jet drops' emitted from the collapse of the unbalanced cavity left by the bubble cap burst (Blanchard 1963; Ghabache \& Séon 2016), and the 'film drops' resulting from the destabilization of the cap itself (Blanchard \& Sysdek 1988; Resch \& Afeti 1991; Spiel 1998).

Lhuissier \& Villermaux (2012a) have depicted the complete evolution of an air bubble formed in a water bulk, from the time it emerges at the liquid surface, up to its fragmentation in dispersed drops. From the description of the drainage of the bubble cap film of tap water bubbles, they discovered that the mechanism of marginal pinching at the bubble foot and associated convection motions in the bubble cap, known as marginal regeneration drive both the bubble cap drainage rate, and are responsible for its puncture. 
Indeed, a bubble is sustained by a surface tension gradient between its foot (where $\sigma$ is smaller) and its pole. Surface tension differences are due to the presence of impurities and surface contaminants of various kinds, in a small amount in water; it is not possible to make a bubble from distilled water, but the water surface is readily contaminated at the contact with ambient air. For a bubble with radius $R$, the cap film thickness $h$ is set by an equilibrium between Marangoni convection moving the liquid upwards, and viscous drainage at the bubble foot. It decays in time $t$ as (Lhuissier \& Villermaux 2012a)

$$
h \sim a\left(\frac{\eta a}{\sigma t}\right)^{2 / 3}\left(\frac{R}{a}\right)^{7 / 3},
$$

a law which is distinct from the drainage law in soap films (Isenberg 1978), or in very viscous bubbles (Debrégeas et al. 1998). The convection motions opposing the viscous drainage of the liquid along the cap also introduce periodically spots of 'dirt' in the sense of Section 4.2.6 over the bubble cap, thus possibly triggering the mechanism discovered by Néel \& Villermaux (2018), for film bursting. It was, along these lines, argued by Poulain et al. (2018) that the celebrated relationship between the cap thickness at bursting $h_{b}$ and the bubble radius

$$
h_{b} \propto \frac{R^{2}}{\mathcal{L}}
$$

discovered by Spiel (1998) could be interpreted from (5.11) as the manifestation of a competition between Marangoni and mixing effects at the bubble foot, leading to

$$
\mathcal{L}=a S c^{2 / 3}
$$

with $S c=\nu / D$ the Schmidt number of the impurities in the water if $D$ denotes their diffusion coefficient.

The mean bubble lifetime $\left\langle t_{b}\right\rangle$ is an increasing function of its radius

$$
\left\langle t_{b}\right\rangle \sim t_{v} \times S c\left(\frac{R}{a}\right)^{1 / 2}
$$

where $t_{v}=\eta a / \sigma$ is a viscous time. Lhuissier \& Villermaux (2012a) have further shown that hole piercing at the bubble foot is a random Poisson process, and determined the (broad) distribution of bubbles lifetimes of which Poulain et al. (2018) have provided extensive measurements in different conditions.

Subsequent to a hole nucleation event, the cap bursting dynamics conditions the resulting spray. Lhuissier \& Villermaux (2012a) have given the details of the processes leading to the (Rayleigh-Taylor) centrifuge destabilization of the hole rim expanding along the curved bubble cap, the mean drop sizes $\langle d\rangle$ and their number $N$ as

$$
\langle d\rangle \sim\left(R^{3} h_{b}^{5}\right)^{1 / 8}, \quad \text { and } \quad N \sim\left(\frac{R}{a}\right)^{2}\left(\frac{R}{h_{b}}\right)^{7 / 8},
$$

as well as the distribution of the droplets sizes emanating from one bubble burst, thus offering, combined with known bubbles production rates over the ocean, an adjustable parameter free prediction for the aerosol flux and spray structure (for droplets $\gtrsim 1 \mu \mathrm{m}$ ) caused by bubble bursting in this precise context (see also Veron (2015) for a collection of recent data).

In sum, natural and ubiquitous water contaminants enable the birth of a bubble and sustain it as it ages, but they ultimately also kill it. Dirtiness thus conditions the entire life of surface bubbles. But if there is a bad, there is also a good dirt regarding the bubbles lifetime expectancy: It was found by Poulain \& Bourouiba (2018) that some specific 

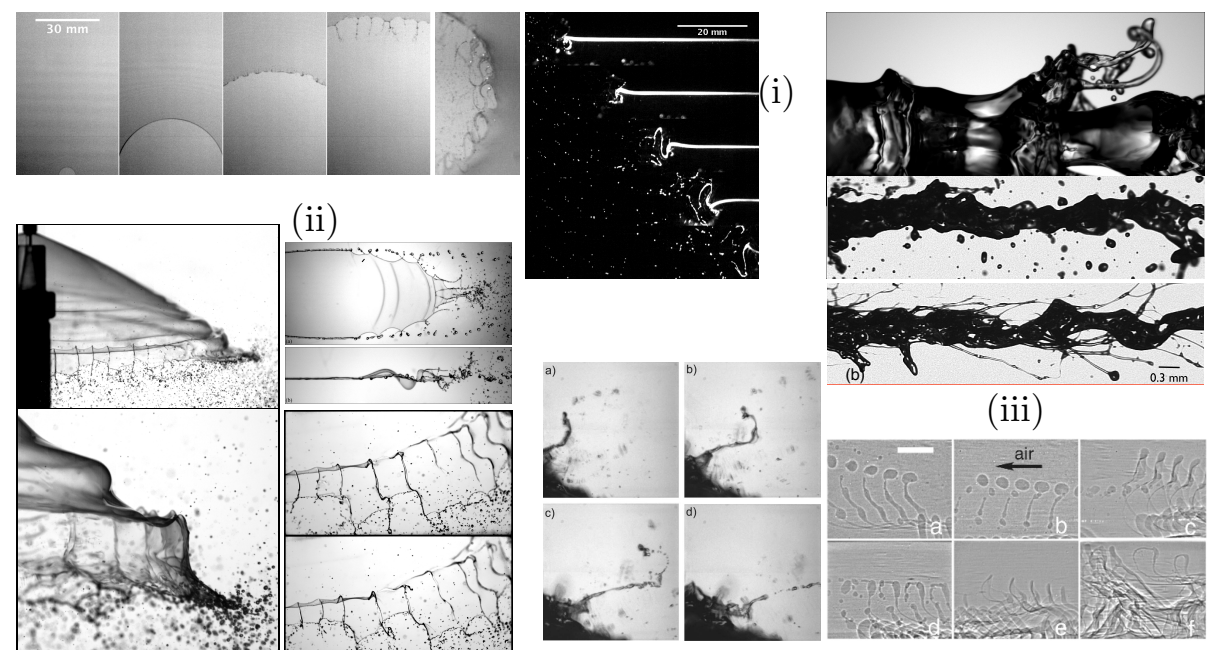

(iii)

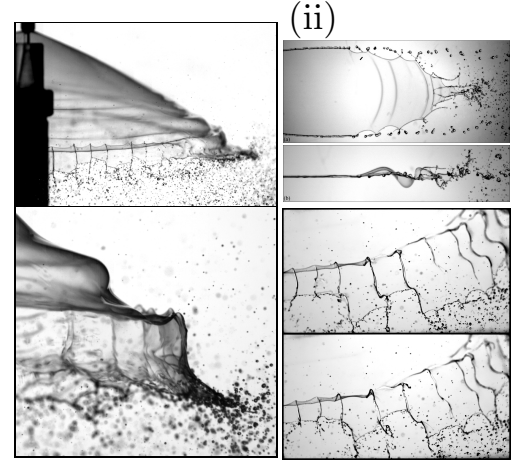

(ii)
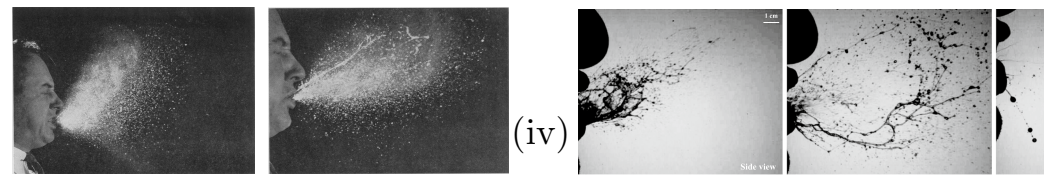

Figure 11. Shear, flags, spray and exhalations. (i) The bursting of soap films involve a flapping Squire instability with the outside gaseous environment (Lhuissier \& Villermaux 2009b). (ii) Sheets (Savart sheets and a plane sheet extruded from a slit) in the Squire flapping régime exhibiting the secondary wavy corridor transverse destabilization mechanism (Bremond et al. 2007; Lhuissier \& Villermaux 2012b). (iii) Liquids sheared by a fast gas stream exhibiting the characteristic ligaments formation at the interface: water-air (Marmottant \& Villermaux 2004b), ocean spray (Veron et al. 2012), a polymeric viscoelastic liquid (Keshavarz et al. 2016), and the phenomenon seen in a dense spray using an X-ray phase-contrast imaging technique (Wang et al. 2008). (iv) Human exhalation from a sneeze: The historical imaging of the phenomenon by $\mathrm{H}$. E. Edgerton at MIT (Turner et al. 1941), and its modern version (Scharfman et al. 2016) on the right.

bacterial secretions in the liquid enhance the lifetime of bubbles, hindering hole nucleation like surfactants do (see also Fig. 6 in Néel \& Villermaux (2018)). Initially, marginal regeneration governs the bubbles cap thinning rate, similarly to water bubbles as in (5.12). However, due to their enhanced lifetime, it is eventually evaporation that governs their thinning, thus also dramatically decreasing the cap thickness at burst (Champougny et al. 2018). Miguet et al. (2020) have nevertheless underlined the importance, in order to lock to this final régime, of working with surfactants exempt from impurities, these triggering the classical Marangoni piercing mechanism described above.

While still in need of a full rationalization, such observations are certainly relevant to understand how very fine particles are introduced in the atmosphere from the ground/sea level.

\section{Gone with the wind: shear, flags, spray and exhalations}

A liquid volume may fragment as a result of a velocity difference with its surrounding environment and indeed, a broad class of 'atomization' processes involve a shear at the interface. The disintegration and dispersion of a liquid volume by a gas stream is a phenomenon which encompasses many natural and industrial operations: The entrainment of spray droplets by the wind over the ocean, the generation of pharmaceutical sprays, 
the dispersion of liquid propellants in combustion engines, or the exhalation of postillions are among the many obvious examples (see Section 1.2).

\subsection{Shear at the interface}

By shear, we do not only mean the simple drag force exerted, for instance, on receding rims opening on soap films at low Reynolds number in a still atmosphere (Mysels \& Vijayendran 1973; Frens 1974; Reyssat \& Quere 2006), we mean something else, a new ingredient responsible for the destabilization of the interface of a liquid volume in relative motion with its environment, triggering a subsequent sequence of events leading to its fragmentation.

The interface separating two initially parallel streams having different velocities $u_{1}$ and $u_{2}$ is naturally unstable: this is the Kelvin-Helmholtz paradigm recalled in Appendix A.3. The shear $\Delta u=\left|u_{2}-u_{1}\right|$ is always destabilizing and, in the absence of other length scales in the problem, destabilization of the interface occurs around a wavelength given by

$$
\lambda \sim \frac{\sigma}{\rho_{2}(\Delta u)^{2}}
$$

where $\rho_{2}$ is the density of the lightest stream. However, the above results only applies in the rare situations where a sharp velocity discontinuity at the interface is a good description. It may be relevant to the initial destabilization of high speed jets issuing into a quiescent environment from a nozzle at very high Reynolds number (Hoyt \& Taylor 1977) and is also found to be a good model for the flapping instability of fast thin liquid sheets for which the boundary layers have no time to form (Section 6.3 below).

For a large class of other systems, the existence of boundary layers at the interface has to be taken into account. Producing a shear between parallel streams implies conveying channels separated by a splitter plate at the wall of which boundary layers form. Therefore, in addition to the intrinsic length scale in (6.1) are the two boundary layer thicknesses $\delta_{1}$ and $\delta_{2}$ of each stream. These two are linked to each other by the continuity of stress at the interface and in practice, only the one in the fastest stream (say, $u_{2}$ ) matters (see Eggers \& Villermaux (2008)). Generalizing Rayleigh's treatment of the shear instability of a smooth velocity profile (see Appendix A.3) to the case with density differences between the phases, Villermaux (1998a) has shown that the most amplified wavelength and its growth rate are (when $u_{2} \gg u_{1}$ )

$$
\lambda \sim \delta_{2}\left(\frac{\rho_{1}}{\rho_{2}}\right)^{1 / 2}, \text { and } \operatorname{Re}(-i \omega) \sim \frac{\rho_{2}}{\rho_{1}} \frac{u_{2}}{\delta_{2}}
$$

provided

$$
\begin{aligned}
W e_{\delta} & =\frac{\rho_{2}(\Delta u)^{2} \delta_{2}}{\sigma} \\
& >\left(\frac{\rho_{2}}{\rho_{1}}\right)^{1 / 2}
\end{aligned}
$$

Mode selection and growth rate are now independent of surface tension but are solely given by the shape of the velocity profile of the fast stream. Even in the presence of surface tension, the shear does not affect the layer for wavelengths shorter than $\lambda$ given in (6.2). The Rayleigh mode selection thus overcomes the selection of the Kelvin-Helmholtz mode as long as $\sigma / \rho_{2} u_{2}^{2}<\delta_{2}\left(\rho_{1} / \rho_{2}\right)^{1 / 2}$, resulting in (6.4) above.

As recalled in Appendix A.3, this instability mechanism of inflexional velocity profiles is inviscid, as opposed to the viscous mechanism of boundary layer profiles. Viscous 
corrections do exist however for $u_{2} \delta_{2} / \nu_{2}$ smaller than about 100 (Betchov \& Szewczyk 1963). They were considered for jets by Gordillo \& Pérez-Saborid (2005), in particular.

\subsection{Viscoelastic fluids}

The case of viscoelastic fluids is however more dramatic (Rallison \& Hinch 1995). As explained by John Hinch in an appendix to Azaiez \& Homsy (1994), large nonNewtonian stresses are confined to the shear layer at the interface only, and thus one anticipates that the stabilizing effect of the normal stresses on the long unstable waves is just that of an elastic membrane or (dynamical, shear sustained) surface tension on the Kelvin-Helmholtz instability. For a viscoelastic liquid with elastic shear modulus $G$ and relaxation time $\tau$, the effective surface tension writes, according to J. Hinch, as

$$
\sigma \sim \frac{G \tau^{2}(\Delta u)^{2}}{\delta_{1}}
$$

in our notations where $\delta_{1}$ stands for the boundary layer thickness in the liquid. The most amplified wavelength and its growth rate are given, Mutatis Mutandis, by (A 20) in Appendix A.3.

The phenomenology of shear instabilities with viscoelastic liquids is qualitatively similar to those with normal fluids (primary shear destabilization, ligaments formation, broad droplets size distribution, see e.g. Keshavarz et al. (2016)), but a systematic experimental study is still lacking. It would, however, be welcome: many exhalations in nature involve non-Newtonian fluids, including human exhalations (Scharfman et al. 2016). The postillions expelled from the mouth at a cough, or sneeze have been torn-off by a rapid air flow from a layer of mucus in the lung, a mucus whose rheological properties are far from Newtonian. Understanding better their mode of production is necessary to make sense of the inherently very broad size distribution of particles sizes released at a sneeze (Duguid 1946; Morawska et al. 2009; Zayas et al. 2012) which has, to date, not received a satisfactory description.

\subsection{Thin films: flapping}

The case of thin fast liquid sheets projected in a gaseous environment at rest is the closest to ideal realization of the pure Kelvin-Helmholtz scenario, albeit in its thin layer limit, which favors a sinusoidal mode of instability, conferring to the sheet a characteristic flapping motion. This is the Squire instability explained in Appendix A.4. The sheets are formed, for instance, by the deflection of a jet impacting a solid surface, or by extruding the liquid through a thin slit, as with standard flat fan agricultural nozzles.

The growth rate of the corresponding Squire instability $t_{\text {squire }}^{-1} \sim \sqrt{\rho_{2} / \rho_{1}} \times u_{1} / \sqrt{\lambda h}$ depends on the sheet thickness $h$, and on the gas to liquid density ratio whereas the most amplified wavelength $\lambda$ is given by (6.1) above (see Appendix A.4 and Hagerty \& Shea (1955); Dombrowski \& Johns (1963); Crapper et al. (1973); Villermaux \& Clanet (2002)).

As for the 'Liquid intact length' of jets in (8.16) in Section 8.2 below, the distance, or 'radius of intact sheet' (for a Savart sheet) is given by $R=u_{1} t_{\text {squire }}$ with $u_{1}$ the conserved liquid velocity. This is the distance it takes for the flapping amplitude motion to be intense enough for a secondary mechanism, involving a thin film Rayleigh-Taylor instability called the 'wavy corridor mechanism' examined below, to operate. This distance reflects the time of growth of the primary undulations amplitude, the second mechanism being comparatively much shorter, hence the proportionality of $R$ to $t_{\text {squire }}$. The breakup radius of a Savart sheet for which $h(R) \sim d_{0}^{2} / R$ is thus, in the Squire régime and in place of 
(i)

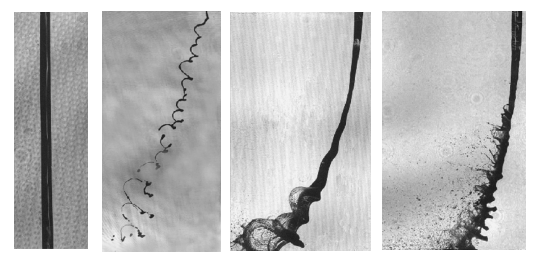

(ii)

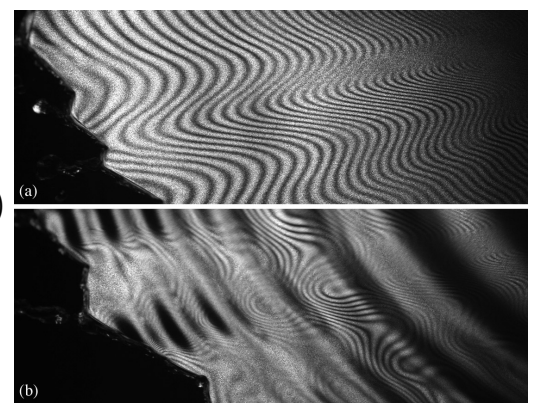

(iii)
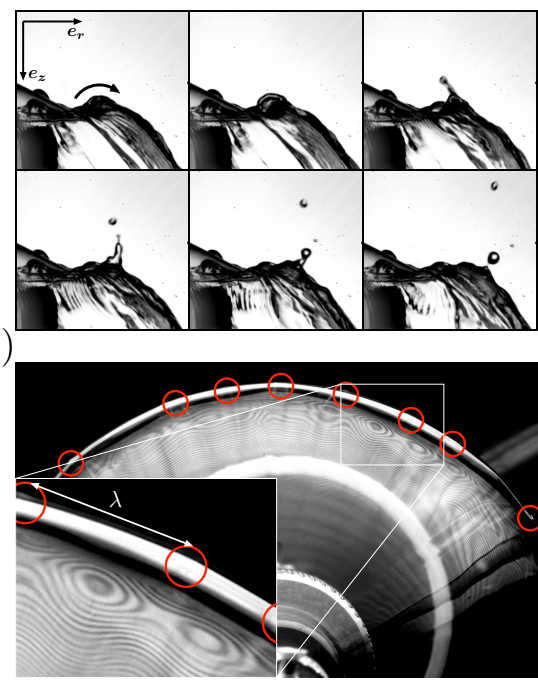

Figure 12. Illustrations of the wavy corridor mechanism: (i) Liquid jet in a transverse cross flow of air. The centripetal acceleration imparted to the liquid flowing along its curved path destabilizes the jet (Sallam et al. 2004). (ii) Undulated Savart sheet in the flapping régime. For large enough amplitudes, primary undulations undergo a transverse destabilization (thickness modulations seen by interferometry) caused by the accelerations imposed to the liquid at the passage of the primary undulations (Bremond et al. 2007). (iii) Evidence for the centrifugation of liquid particle at the passage of a sharp cusp on a crumpled Savart bell, and the consequence of this acceleration on the transverse thickness field of the sheet (Lhuissier \& Villermaux 2012b).

$$
\frac{R}{d_{0}} \sim\left(\frac{\rho_{2}}{\rho_{1}}\right)^{-2 / 3} W e^{-1 / 3}
$$

holding for $W e \gtrsim 40 \sqrt{\rho_{1} / \rho_{2}}$ of order $10^{3}$ for a water sheet moving in air (density ratio $\rho_{2} / \rho_{1} \approx 10^{-3}$ ) with velocity $u_{1}$ (Huang 1970; Villermaux \& Clanet 2002).

To the primary Squire undulated pattern of the sheet is superimposed, via the mechanism described next in Section 6.4, a thickness modulation field. On a bounded liquid sheet, these thickness modulations induce a differential recession speed at their edge, which may lead to a periodic cutting of the sheet into periodic spanwise strips of width $\lambda$ given in (6.1) above (see Fig. 9 and Kim \& Sirignano (2000)). These strips further recoil into a ligament of diameter

$$
\langle d\rangle \sim \sqrt{\lambda h}
$$

which also sets the average droplets sizes formed by this process, with viscous corrections when applicable (Dombrowski \& Johns 1963; Han et al. 2004; Kooij et al. 2018).

\subsection{The wavy corridor mechanism}

The shear instability generates the characteristic waves extending spanwise, perpendicular to the direction of the mean flow with a wavelength given either by (6.1) or by (6.2) depending on $W e_{\delta}$. It is the first step of the inertial destabilization of the interface, leading to its fragmentation. The second step consists in the destabilization of these primary waves themselves, through a mechanism we explain now.

As they grow in amplitude, the primary shear waves destabilize in the spanwise direction to form streamwise ligaments separated by a well defined wavelength $\lambda_{\perp}$. The mechanism responsible for this secondary instability was first proposed by Marmottant 
\& Villermaux (2004b) to describe the fragmentation of a slow liquid stream (density $\rho_{1}$ ) by a fast gas stream (density $\rho_{2}$ ). The fundamental idea exploits the fact that, in shear instabilities, the group velocity $u_{c} \approx u_{2} \sqrt{\rho_{2} / \rho_{1}}$ of the most amplified perturbation is intermediate between the velocity of the slow stream $u_{1}$ and that of the fast stream $u_{2}$. Fluid particles in the slow stream are thus periodically accelerated perpendicular to the interface (as if transported in a wavy corridor) which, therefore, suffers a Rayleigh-Taylor instability. In the reference frame of the liquid, the interface with elevation $\xi \sim a \sin (\omega t)$ experiences an oscillatory acceleration of intensity

$$
|g| \sim a \omega^{2}
$$

with $\omega \sim u_{c} / \lambda$ and $a$ the amplitude of the primary shear waves. The transverse instability of the primary wave crests occurs when the Rayleigh-Taylor instability timescale $\left(\sigma / \rho_{1}|g|^{3}\right)^{1 / 4}$ (see (A 8) in Appendix A.1) gets shorter than the residence time of the waves $\omega^{-1}$, setting both the critical amplitude $a_{c}$ and transverse wavelength $\lambda_{\perp}$ as

$$
\begin{aligned}
\frac{a_{c}}{\lambda} & \sim \frac{\lambda_{\perp}}{\lambda} \sim W e_{\lambda}^{-1 / 3} \\
\text { where } \quad W e_{\lambda} & =W e_{\delta} \times \frac{\lambda}{\delta}
\end{aligned}
$$

From the resulting protrusions of the -now indented- waves crests emerge liquid ligaments which are further accelerated in the fast gas stream where they fragment in stable droplets. The volume of the ligaments is of order $\lambda_{\perp}^{3}$ and they typically produce broad droplets size distributions with average size $\langle d\rangle \sim 0.1 \lambda_{\perp}$.

This process is sometimes called shear assisted atomization in the context of liquid propulsion engines. It is responsible for the formation of sea spray by strong winds over sharp crested waves (Anguelova \& Barber 1999; Veron et al. 2012).

The above wavy corridor mechanism operates more generally when a liquid flows through a curved path and experiences therefore a centripetal acceleration. As such, it also applies to liquid films and sheets. Depending on the intensity of the centripetal acceleration and on the thickness of the film, either the infinite depth (Appendix A.1), or the finite thickness (Appendix A.2) versions of the Rayleigh-Taylor instability apply. This mechanism explains the cellular fragmentation of liquid jets bent in a cross-flow (see the experiments by Sallam et al. (2004) and the corresponding theory in Section 3.11.3 of Eggers \& Villermaux (2008)), the transverse indentations of undulated liquid sheets (Bremond et al. 2007) and the fragmentation of soap films by the transverse instability of their flapping receding rim (Lhuissier \& Villermaux 2009b), as well as the spontaneous burst of crumpled water bells (Lhuissier \& Villermaux 2012b). It is distinct from the mass concentration mechanism in a vibrating, non-flowing soap film discussed by Boudaoud et al. (1999).

\section{Impacts}

Impacts between cohesive bodies sometimes result in their fragmentation. The role of the impact is two-fold: it may first simply communicate to the body a stress sufficient to overcome cohesion, as in the generic example of the magnets necklace in Section 2.3. This is often how solids break. Second, an impact may also trigger a shape change of the body to make it sensitive to an intrinsic instability. This is the case with liquids where fragmentation under impact is mediated by the transient formation of ligaments, which naturally breakup into drops (Section 8). Note that this distinction is not exclusive, since the fragmentation of some slender solid bodies by impact does involve the development 


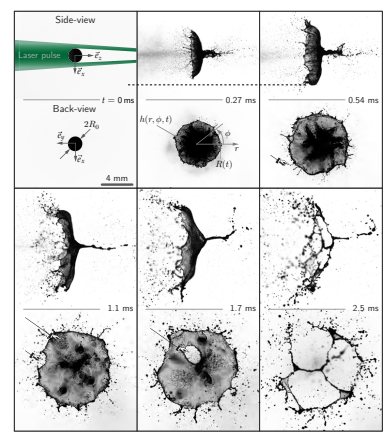

(i)

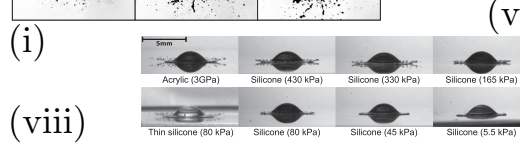

(viii)

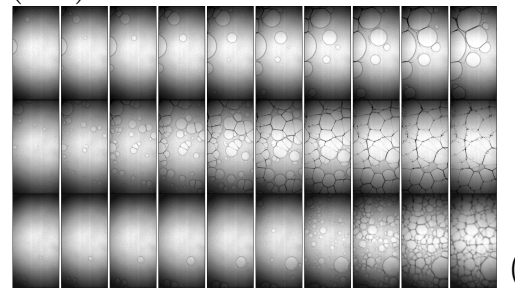

(ii)

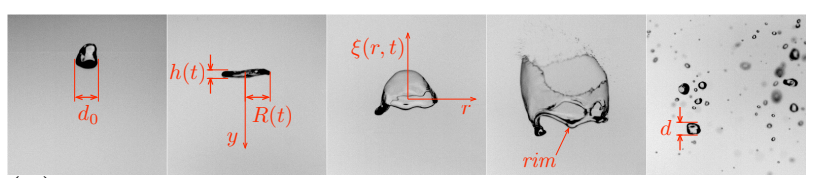

(iii)

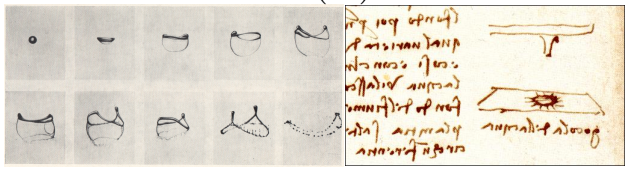

( $\mathrm{v})$

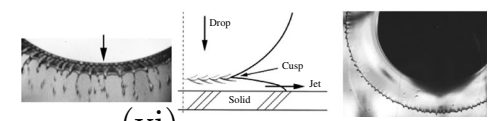

(vi)

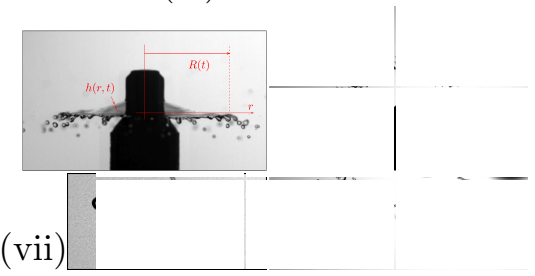

$\odot$

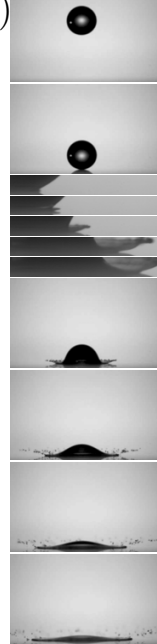

Figure 13. Hard and soft impacts. (i) Impact of a laser pulse on a liquid drop which flattens, expands, is transpierced by holes, and fragments (adapted from Klein et al. (2020)). (ii) Series of events of the fragmentation of a $d_{0}=6 \mathrm{~mm}$ water drop falling in an ascending air stream showing the flattening of the drop into a pancake shape with radius $R(t)$ and thickness $h(t)$, the inflation of a bag with shape $\xi(r, t)$ bordered by a thicker corrugated rim, whose breakup leads the final drop sizes $d$ (Villermaux \& Bossa 2009). (iii) Two historically important pictures featuring the soft impact of a drop deforming in air (Lane \& Green 1956), and the drawing of a water drop crushed on a flat hard substrate by Leonardo da Vinci (da Vinci 1508). (iv) Impact of a drop on a solid substrate (adapted from (Riboux \& Gordillo 2015)). (v) Drop impact on gradually softer materials, and rarefied ambient pressures (Howland et al. 2016). (vi) Micro-splashing from the first ejecta-sheet at drop impact a solid (Thoroddsen et al. 2012a). (vii) A water drop impaling on a solid target, expanding and featuring multi-sized radial ligaments at its rim, from which drops form (Villermaux \& Bossa 2011). (viii) Time series of liquid films impacted by a shock wave for three different Mach numbers $M=1.03,1.07$ and 1.21 pierced by holes colliding to form ligaments webs (Bremond \& Villermaux 2005).

of an instability (buckling, see e.g. Gladden et al. (2005)). We describe below several examples of liquid bodies like drops (the archetype of the stable shape) which fragment once impacted, along with the mechanisms of their shape change into ligaments.

\subsection{Hard impacts}

By 'Hard', we mean a cohesive but deformable object (a liquid volume) impacted by another rigid object, the paradigm of this being the crushing of a drop on a solid surface.

\subsubsection{Drop impact on solid surface}

Among the relevant studies documenting fragmentation on impacts are those of Ashgriz \& Poo (1990) and Qian \& Law (1997) who have quantified the conditions for coalescence or satellite formation in binary collisions of drops, Stow \& Stainer (1977) who have measured the number of fragments of a water drop colliding with various solid surfaces, dry, wet, smooth and rough, and measured their size distribution, Mundo et al. (1995) who performed similar measurements with liquids of different surface tension and viscosity, Yarin \& Weiss (1995) who measured the fragment size distributions of a drop impacting 
a shallow layer of the same fluid, Roisman et al. (2006) who made various measurements on the spray resulting from an impact on a dry solid, and $\mathrm{Xu}$ et al. (2007) who singledout the importance of the solid surface texture, and ambient gas conditions on splashing and resulting fragments distribution while more recently Thoroddsen et al. (2012a) and Riboux \& Gordillo (2015) or Wang \& Bourouiba (2018) measured some ejection velocities and drops sizes, and Burzynski et al. (2020) also some distributions.

As for all fragmentation processes, a broad collection of fragment sizes is produced following drop impact, with a typical positively skewed size distribution presenting many small drops, and fewer larger drops. Despite a recent important activity on the topic (drops impacts on hydro-phillic, -phobic, textured, rough, heated etc... surfaces), the remark made by Stow \& Stainer (1977) more than 40 years ago is still largely topical:

Almost without exception, recent investigations of splashing have concentrated on

the observation of the hydrodynamics of the impact itself with little or no attention

being paid to the final consequences of the event.

Fragmentation indeed remains the poor cousin of drops impact. For instance, there is not a single section devoted explicitly to this aspect in the recent review by Josserand \& Thoroddsen (2016). This is not new; as noted by Rowlinson (2002) (p. 56):

...the study of cohesion failed to prosper in the 18th century under the internal difficulties of its own subject matter and the external competition of other more exciting branches.'Everything has its fashions, even philosophy has its own', wrote Réaumur in 1749 , and cohesion became an unfashionable subject for many of the leading figures of the day.

The problem can however be apprehended with relatively simple guidelines: we consider a droplet with radius $r_{0}$ impacting at speed $u_{0}$ normally onto a rigid, flat surface which absorbs its momentum, and redistributes it radially in an axi-symmetrical fashion, into an expanding sheet. The Savart sheet in Section 3.2.3 is the strict analogue, continuous and steady version of this problem. Because they resort to slightly different phenomena, we distinguish two régimes, one before the crushing time $r_{0} / u_{0}$, and the other after, starting with the latter.

(i) After the crushing time $\left(t>r_{0} / u_{0}\right)$ : the main droplets

After the drop has crushed on the solid (this steps lasts for about $r_{0} / u_{0}$ ), its volume $\Omega=\frac{4}{3} \pi r_{0}^{3}$ is entirely converted into a circular sheet with radius $R(t)$. Note that a spherical drop deposited on a surface at zero velocity keeps its shape, and that its initial radius $r_{0}$ is not affected. Hence, the effect of an impact at velocity $u_{0}$ results for $R(t)-r_{0}$ in a correction $F\left(u_{0}, t, \sigma \ldots\right)$ which must vanish for $u_{0} \rightarrow 0$. In other words, The expanded drop radius $R(t)$ must write

$$
R(t)=r_{0}+F\left(u_{0}, t, \sigma \ldots\right) .
$$

The interesting case is obviously not the limit $u_{0} \rightarrow 0$, but rather when the effect of the impact results in a strong shape change with a maximal radius extension $R_{\max } \gg r_{0}$. In that case, the liquid constitutive of the drop shapes into a radially expanding, and recoiling sheet, whose typical thickness $h$ is such that the aspect ratio $h / R$ is very small. We call $u(r, t)$ and $h(r, t)$ the radial velocity and thickness of the expanding drop (Fig. 13), for which we write the Euler equations in the slender slope approximation $\left|\partial_{r} h(r, t)\right| \ll 1$,

$$
\begin{aligned}
\partial_{t} u+u \partial_{r} u & =0 & & \text { Ballistic motion, } \\
r \partial_{t} h+\partial_{r}(r u h) & =0 & & \text { Mass conservation. }
\end{aligned}
$$

We do not consider any interaction with the surrounding environment, nor viscous effects, 
a limit which suits to the configuration of a drop impacting a solid target matching its own size in opened air, ingeniously pioneered by Rozhkov et al. (2002).

The pressure $p(r, t)$ in the liquid is essentially zero after the crunching time. At the impact point in $r=0$, it is roughly

$$
p(0, t) \approx \rho u_{0} r_{0} \delta(t),
$$

the Dirac Delta contribution $\rho u_{0}^{2} \delta(t) \times\left(r_{0} / u_{0}\right)$ standing for the duration of the momentum transfer giving rise to the pressure surge, the source of the sheet radial motion. It lasts for the crushing time $r_{0} / u_{0}$ which is safely taken as zero provided the resulting overall evolution of $R(t)$ lasts for a time much larger than $r_{0} / u_{0}$, as is checked a posteriori.

We seek for a time-dependent solution of the form (Villermaux \& Bossa 2011)

$$
h(r, t)=\frac{f(t)}{r}, \quad \text { and } \quad u(r, t)=-\frac{\dot{f}}{f} r
$$

where $\dot{f}$ denotes $\mathrm{d} f(t) / \mathrm{d} t$. The solution for $R(t)$ is constrained by two conditions which express that

(a) the radially expanding fluid particles are arrested at some distance from the impact point $R(t)$ by capillary confinement,

(b) these fluid particles feed the sheet rim where they progressively all collect.

Global mass conservation thus writes

$$
\int_{0}^{R} 2 \pi r h(r, t) \mathrm{d} r=\Omega-\int_{0}^{t} 2 \pi R(t)(u(R, t)-\dot{R}) h(R, t) \mathrm{d} t
$$

where the second term in the right hand side of (7.6) is the net volume accumulated at time $t$ in the rim.

Momentum conservation at the rim approximately complies to the standard TaylorCulick's law (modulo a time-varying correction involving $\ddot{R}$ which we discard for clarity)

$$
\rho h(R)(u(R, t)-\dot{R})^{2} \approx 2 \sigma
$$

from which, using Eqs. (7.5) and (7.6), we have

$$
f(t) R(t)=\frac{\Omega}{2 \pi}\left(1-\frac{t}{\tau}\right)^{2}, \quad \text { with } \quad \tau=\sqrt{\frac{\rho \Omega}{\pi \sigma}} .
$$

The ballistic motion in the absence of pressure gradient along $r$ expressed by (7.2) translates, given the form of $u(r, t)$ chosen in (7.5), to $f(t)=A / t$ with $A=\Omega /\left(2 \pi u_{0}\right)$ a (dimensional) constant whose value is set by the initial sheet expansion velocity, given by $\dot{R}(0)=u_{0}$ (see next paragraph, where we detail the crushing dynamics itself). The sheet radius is finally

$$
R(t) \sim u_{0} \tau \frac{t}{\tau}\left(1-\frac{t}{\tau}\right)^{2}
$$

describing an asymmetric one-period oscillatory motion lasting $\tau$, and providing a representation for the motion within the sheet $u(r, t)$ and of its thickness $h(r, t)$ as

$$
u(r, t)=\frac{r}{t}, \quad h(r, t) \sim \frac{\Omega}{u_{0} r t},
$$

relations which were found to be adequate by, successively, Villermaux \& Bossa (2011), Vernay et al. (2015b) and Wang \& Bourouiba (2017) for $t \gg r_{0} / u_{0}$. The maximal 
amplitude of the motion, reached at $t / \tau=1 / 3$, is

$$
R_{\max } / r_{0} \sim \sqrt{W e}
$$

where the impact Weber number is $W e=u_{0} \tau / r_{0}$, a well known scaling dependency (see e.g. Chandra \& Avedisian (1991); Rozhkov et al. (2002); Roisman et al. (2002); Mehdizadeh et al. (2004); Ukiwe \& Kwok (2005)). Two remarks are in order:

(a) This model for $R(t)$ holds provided the Weber number $\sqrt{W e} \sim \tau /\left(r_{0} / u_{0}\right)$ is large, expressing a clear separation between the drop crushing time (lasting $r_{0} / u_{0}$ ), and the comparatively long inertia-capillary expansion and recoil dynamics of the deformed drop (lasting $\tau$ ).

(b) Although the scaling in (7.11) can be derived equating kinetic energy with surface energy, the evolution of $R(t)$ is highly dissipative. Dissipation does not occur at the impact location, nor in the sheet whose motion is conservative, but at the rim where all the incoming kinetic energy of the particles arrested at the rim is absorbed. It is overall a fixed fraction of the initial drop kinetic energy (see Section 11).

The kinematics of $R(t)$ explains why and how the liquid initially constitutive of the drop, which progressively accumulates in a toroidal ligament (the sheet rim), will degenerate into radial ligaments (first witnessed by da Vinci (1508), see Fig. 13 and also Marmanis \& Thoroddsen (1996)), finally forming drops.

The fundamental ingredient is that the rim sits on a non-Galilean frame which is ever decelerated $(\ddot{R}<0$ until $t / \tau=2 / 3$, see (7.9)), thus forcing the radial expulsion of liquid masses with respect to the rim, through a Rayleigh-Taylor like process (see Allen (1975) and Appendix A.1). The rim, because of its toroidal shape, is primarilly sensitive to the familiar capillary instability, the very one responsible for its destabilization (see references in Section 4.1 and Zhang et al. (2010)), which occurs however here on a stretched substrate (see Section 8.2 ) because the radius $R(t)$ expands, with stretching rate $\dot{R} / R$. Once articulated together, these ingredients provide quantitative predictions to understand the fragmentation scenario: the body force $-\rho \ddot{R}$ pushes the unstable bulges constitutive of the rim outwards at a lengthscale matching the rim's diameter, thus forming the radial ligaments whose breakup produce a droplets size distribution characteristic of very corrugated ligaments (see Fig. 13 and Section 9). The average droplet size is

$$
\frac{\langle d\rangle}{r_{0}} \sim W e^{-1 / 4}
$$

Of course, in this his slowed down, expanding rim destabilization mechanism, ligament shedding occurs from the start (Wang \& Bourouiba 2018) when $-\ddot{R}$ is maximal, and proceeds all the way via the turning point of the sheet radius trajectory (when $\dot{R}=0$, as the stretching cancels) when most of the liquid has pilled-up in the corrugated rim, up to the cancellation of expulsion force when $\ddot{R}=0$; Fragmentation is thus typically completed at $t / \tau \approx 2 / 3$.

(ii) Before the crushing time: ejecta sheet and 'fines'

We have explained in Section 3.2.1 that because viscous dissipation occurs at a very small scale when $O h \ll 1$, the momentum transfer at a collision may lead to the formation of tiny objects, which may also be formed before the drop crunch is completed (i.e. for $\left.t<r_{0} / u_{0}\right)$. We analyze this fine-grained aspect of the phenomenon here, exploring what happens 'inside' the Dirac Delta of the pressure surge mentioned in the previous section. Two régimes are distinguished:

1) The geometrical penetration radius of a spherical droplet on a rigid floor is initially 
$a \sim \sqrt{u_{0} r_{0} t}$. The induced flow $\mathbf{u}(\mathbf{r}, t)$ ruled by $\partial_{t} \mathbf{u}+\mathbf{u} \cdot \nabla \mathbf{u}=-\nabla p / \rho$ and $\nabla \cdot \mathbf{u}=0$ obeys initially, when the velocity amplitude $|\mathbf{u}|$ is small enough to neglect the cross-term $\mathbf{u} \cdot \nabla \mathbf{u}$ (see Lamb (1932), Art. 11 and Cooker \& Peregrine (1995))

$$
\begin{aligned}
\partial_{t} \mathbf{u} & =-\frac{1}{\rho} \nabla p, \\
\text { and thus } \quad \nabla^{2} p & =0 .
\end{aligned}
$$

In other words, if the liquid has moved over a distance $a$ in the $r$-direction, then it has moved over the same distance in the direction perpendicular to the impact plane. The consequence of the Laplacian character of the pressure is that since $a$ is the only initial lengthscale of the problem (besides $r_{0} \gg a$ ), the net volume of liquid whose motion is slowed down is of order $a^{3}$, setting its mass $m \sim \rho a^{3}$ (not to be confused with the deflected mass $\rho u_{0} t a^{2}$, feeding a radially ejected lamellae). The cancellation of the corresponding momentum initially carried in the impact direction gives rise to a force $f=u_{0} \dot{m}$, and therefore to an isotropic pressure at the impact point given by

$$
\begin{aligned}
p(0) & \sim \frac{f}{a^{2}} \\
& \sim \rho u_{0}^{2} \sqrt{\frac{r_{0}}{u_{0} t}},
\end{aligned}
$$

an early time divergence familiar in impact problems (Wagner 1932; Cointe \& Armand 1987; Philippi et al. 2016). The pressure gradient $\partial_{r} p$ in the radial direction is of order $p(0) / a$ so that, from the dynamics in $(7.13), u / t \sim u_{0} / t$, giving simply

$$
u \sim u_{0}
$$

hence the initial condition $\dot{R}(0)=u_{0}$ leading to the sheet trajectory in (7.9) after the crushing time.

The rate of increase of the mass in motion $\dot{m}$ is proportional to the driving force $p(0) \times a^{2}$, so that the velocity is constant. With this estimate for $u$, the amplitude of the discarded nonlinear term $|\mathbf{u} \cdot \nabla \mathbf{u}|$ in the Euler equation above is of order $u_{0}^{2} / a \sim 1 / \sqrt{t}$, indeed smaller than $1 / t$ as $t \rightarrow 0$. The intensity of this induced flow is smaller than the geometrical expansion velocity of the penetration region $\dot{a} \sim \sqrt{u_{0} r_{0} / t}$ as long as $t<r_{0} / u_{0}$, consistent with the empirical observation that a lamellae is seen to emerge from the impact region when the penetration distance is a fraction of $r_{0}$, and that the ejection velocity of the resulting lamellae (and detached droplets), is of order $u_{0}$ when complications with liquid viscosity, ambient medium, and substrate roughness are negligible (see Xu et al. (2007); Riboux \& Gordillo (2015)).

2) The linear pressure impulse solution in (7.13) does not, however, apply everywhere in the penetration region. Close to the contact line between the drop and the solid, the radial velocity $u$ is itself of order $\dot{a}$, making the nonlinear term $|\mathbf{u} \cdot \nabla \mathbf{u}|$ of order $u^{2} / \delta(t)$ where $\delta(t)$ is some lengthscale setting the width of the pressure and velocity gradients close to the contact line. If $\delta(t)$ is itself initially zero and an increasing function of time, the nonlinear term is more singular than $u_{0}^{2} / t$, suggesting that the early time dynamics balances inertia with pressure, at least in a small region of size $\delta$. Writing $p \sim f /(a \delta)$ so that $\partial_{r} p \sim f /\left(a \delta^{2}\right)$, the balance between $|\mathbf{u} \cdot \nabla \mathbf{u}|$ and $|\nabla p| / \rho$ at the contact line writes

$$
\begin{aligned}
\frac{u^{2}}{\delta} & \sim \frac{1}{\rho} \frac{f}{a \delta^{2}}, \quad \text { or } \quad u \sim u_{0} \frac{a}{\delta}, \\
\text { providing } \delta & \sim u_{0} t, \quad(\text { since } u \sim \dot{a})
\end{aligned}
$$


so that the local pressure in the contact line region (Mandre et al. 2009) is now of order $f /(a \delta) \sim \rho u_{0} r_{0} / t$, indeed more singular than $p(0)$ in (7.16). Detailed calculations (Birkhoff et al. 1948; Riboux \& Gordillo 2014) show that the contact line velocity is closer to $u=2 \dot{a}$ (see Howinson et al. (1991), and Philippi et al. (2016) for a fully self-similar description), albeit affected by viscous corrections when a non-slip condition applies at the solid surface. Expressing that the deflected mass $\rho u_{0} t a^{2}$ all enters the ejected lamellae which carries its momentum provides the lamellae thickness $h$ as $u_{0} \partial_{t}\left(\rho u_{0} t a^{2}\right) \sim \rho u^{2} a h$, that is $h \sim\left(u_{0} / r_{0}\right) a^{2} / \dot{a} \sim t^{3 / 2}$ (see e.g. Riboux \& Gordillo (2014); consistently $h<\delta$ as $t \rightarrow 0)$.

This very early dynamics produces a fast $(\dot{a} \sim 1 / \sqrt{t})$, thin $\left(h \sim t^{3 / 2}\right)$ lamellae, sometimes called 'first-ejecta sheet' (Fig. 13) which, because it is decelerated by the necessary capillary confinement $(\ddot{R}<0$ as above) produces tiny droplets by a process similar to the one described for the main droplets after the crushing time. This régime prevails as long as $\delta<a$, that is $t<r_{0} / u_{0}$, then leaving place to the one in (7.17). The small droplets are called 'fines' in contexts where they are feared for their long persistence in air, and associated large contamination radius when they are drifted by the wind (see Section 1.3). Their occurence has been precisely documented by Néel et al. (2020) for the collision of liquid rims in the effervescent atomisation process.

\subsubsection{Liquid film hit by a shockwave}

Liquid sheets, especially when they are embedded in an explosion (Section 5.1.1), or are flowing rapidly in a gas phase in which they flap (Section 6.4), suffer accelerations perpendicular to their plane. We have emphasized the importance of this phenomenon on their destabilization, through thickness modulations, prelude of holes nucleation (Appendix A.2).

A paradigm of this phenomenon is the case of a soap film impulsively accelerated perpendicular to its plane, when hit by a parallel shockwave in air (qualitatively similar observations are made when the film is curved, like for a bubble, see Layes et al. (2003)). The shock strength is measured by the Mach number $M=v / c$, the ratio of the shock velocity $v$ to the sound speed $c$. Since the acoustic impedance of water is much larger than that of air, the shock is nearly completely reflected at the liquid film surface, a rebound which communicates an impulse setting the film into motion. Elementary gas dynamics (Henderson 1989) provides the film velocity $u(t)$ and the acceleration time $\tau$ as

$$
\begin{aligned}
u(t) & =u\left(1-\frac{e^{-t / \tau}}{2\left(M^{2}-1\right)}\right), \quad \text { with } \quad \frac{u}{c}=\frac{2\left(M^{2}-1\right)}{2 \gamma M^{2}-\gamma-1} \\
\tau & =\frac{\rho h c}{P} \frac{\gamma+1}{2 \gamma M^{2}-\gamma+1}
\end{aligned}
$$

where $P$ is the gas ambient pressure in front of the shock and $\gamma \approx 1.4$ for air. The final velocity coincides with the gas velocity behind the shock, while the acceleration time, reflecting the liquid inertia, depends on the film thickness $h$.

Even at a moderate Mach number $M$ of order unity, the acceleration time $\tau$ is, for a few microns thick film, extremely short (of the order of $10^{-5} \mathrm{~s}$ ), much shorter than the development time of the thin-film Rayleigh-Taylor instability responsible for its burst. The acceleration $g$ (in the notation of Appendix A.2) is thus essentially impulsive

$$
g=u \delta(t)
$$

and a transient gain analysis (because the acceleration is, strictly speaking, time dependent) predicts the wavelength whose amplitude matches the film thickness $h$, thus piercing 
the film into a collection of holes, as a function of $M$ along with the time it takes to do so (see Fig. 13). Holes are denser in the film plane, and nucleate sooner for a stronger shock (Bremond \& Villermaux 2005). This academic setup, which confronts precise predictions with a simple - yet not straightforward- especially designed experiment, demonstrates the importance of the thin-film limit description of liquid sheet stability.

\subsubsection{Drop hit by a laser pulse}

A fundamentally very similar problem to the one described above (although directly motivated by the extreme ultraviolet light production for nanolithography, see Klein et al. (2015)) consists in shooting within a very short time an intense laser pulse on a drop of light-absorbing liquid. Part of the injected light energy vaporizes a fraction of the liquid drop mass which, as it is released from the drop surface at the thermal velocity propels, by momentum conservation, the rest of the drop at constant velocity.

The corresponding surface normal stress deforms the drop into a pancake shape which expands radially and thins very much like a drop impacting a solid does. At the same time the sheet expands, Rayleigh-Taylor modes corrugate its thickness for the same reason explained in Section 7.1.2, and its rim destabilizes for the reason explained in Section 7.1.1 above.

Eventually, the resulting liquid sheet is thus fragmented by at least two distinct processes, the rim shedding ligaments breakup process, and a holes-mediated, 'effervescent' like process (Section 4.4). The study of Klein et al. (2020) also underlines the deterministic role played by inhomogeneities in the laser beam on the final perforation pattern of the expanded liquid sheet.

\subsection{Soft impacts}

By 'Soft', we mean a cohesive but deformable object (a liquid volume) impacted by, or projected into, another medium which deforms as well; the paradigm of this being the formation of raindrops.

\subsubsection{Raindrops}

Like many natural objects, raindrops are distributed in size. By extension of what is known to occur inside the clouds, where small droplets grow by accretion of vapor and coalescence, raindrops in the falling rain at the ground level were believed to result from a complex mutual interaction with their neighbors. Villermaux \& Bossa (2009) suggested that the raindrops polydispersity, generically represented according to Marshall-Palmer's law (Marshall \& Palmer 1948), can be quantitatively understood from the fragmentation products of non interacting, isolated drops. Both the shape of the drop size distribution, and its parameters are related from first principles to the dynamics of a single drop deforming as it falls in air, ultimately breaking into a dispersion of smaller fragments containing the whole spectrum of sizes observed in rain.

Large liquid globules fall by their own weight from the base of the clouds (Hobbs \& Rangno 2004), experiencing a drag resistance from the air, the deforming medium in which they fall. This net drag force results from an inhomogeneous pressure repartition at the surface of the globule, which thus deforms in a pancake shape whose radius $R(t)$ is only limited by capillary confinement. From an analysis very similar to the one in Section 7.1.1 (which also proved to be useful for drops hit by a laser pulse, see Section 7.1 .3 above and Gelderblom et al. (2016)), it is easily shown that the dynamics of $R(t)$ is ruled by

$$
\frac{\ddot{R}}{R}=\frac{1}{\tau^{2}}\left(1-\frac{6}{W e}\right)
$$


where $\tau$ is a characteristic time familiar in the aerodynamics of drops (Ranger \& Nicholls 1969) and $W e$ is based on the air density $\rho_{a}$, free-fall velocity $U \sim \sqrt{\left(\rho / \rho_{a}\right) g d_{0}}$ and size of the globule $d_{0}$ as :

$$
\tau=\frac{d_{0}}{2 U} \sqrt{\frac{\rho}{\rho_{a}}}, \quad \text { and } \quad W e=\frac{\rho_{a} U^{2} d_{0}}{\sigma} .
$$

Viscous corrections can be incorporated in (7.23), but are superfluous for a low viscosity liquid such as water and millimetric drops (Kulkami \& Sojka 2004).

The maximal size $d_{\max }$ of a stable drop at terminal velocity in quiescent air for which both $U=\sqrt{\left(\rho / \rho_{a}\right) g d_{\max }}$ and $W e=6$ (see 7.23) is

$$
d_{\max }=a \sqrt{6}
$$

with $a \sim \sqrt{\sigma / \rho g}$ the capillary length, giving $d_{\max } \approx 6 \mathrm{~mm}$, a value which indeed coincides with the cut-off sizes recorded in natural rain (Marshall \& Palmer 1948; Mason 1971). Larger globules bursts in a catastrophic manner (Fig. 13), involving the inflation of a bag bordered by a thick rim which collects most of the liquid, and whose breakup forms a continuous, exponential-like $\left(\sim e^{-d /\langle d\rangle}\right)$ distribution of fragments sizes (see Section 9) parametrized by its mean $\langle d\rangle$, called the Marshall-Palmer distribution.

It was known since Bentley (1904), and von Lenard (1904) that the size distribution steepness $\langle d\rangle^{-1}$ is solely related to the rate of rainfall $\mathcal{R}$ (typically measured in millimeters per hour): drops sizes are more broadly distributed in heavy storms than in fine mists. Villermaux \& Bossa (2009) have shown, on the basis of the above single drop fragmentation scenario supplemented by a simple mass balance, that these two quantities are related to each other by

$$
\langle d\rangle^{-1} \sim \mathcal{R}^{-\frac{2}{9}}
$$

thus giving a status to the scaling exponent 0.21 originally measured by Marshall \& Palmer $(2 / 9=0.222 \ldots)$. This scenario also gave the clue to rationalize the puzzling observation that apparent fall velocities of some drops can be larger than the terminal velocity expected from their size (Villermaux \& Eloi 2011).

Of course, raindrops occasionally collide as they fall towards the ground. However the topological change from a big drop into smaller stable fragments - the raindrops- is accomplished within a timescale much shorter than the typical collision time between the falling drops. One swallow does not a summer make: The effects of these rare, decorative events is completely screened by the single drop, spontaneous breakup phenomenon, which in itself contains the whole spectrum of drop sizes (Villermaux \& Bossa 2010).

\subsubsection{Impact on deformable surfaces}

There is a continuum of substrate stiffnesses between the rigid floor used for standard (hard) drop impact, and the dilute gas of the raindrop (soft) problem above. The collision of a drop with a layer of the same liquid is an intermediate case between hard and soft. Starting from the hard side, Howland et al. (2016) have interestingly considered gradually softer materials by varying the level of cross-linkage of silicone gels. Splashing of a liquid drop is found to be delayed on a softer substrate, which deforms like a spring as long as the peak pressure in the contact line region $p \sim \rho r_{0} u_{0} / t$ at early time (see Section 7.1.1, (ii), 2)) is larger than its Young modulus $E$. This damping effect delays and slows down the ejecta sheet, which may even be suppressed (Fig. 13). Very deformable substrates like soap films prevent splashing completely (Courbin \& Stone 2006). Wet dense granular materials like quicksand behave, when impacted, either as a liquid, or as a brittle solid depending on their initial packing fraction (Soundar Jerome et al. 2016). 


\subsection{Chemical synthesis by impact}

Impacts are clearly associated with fragmentation, with the dismantling of macroscopic ensembles, but less obviously with the synthesis of complex architectures from elementary pieces. This is only true at first sight since we have already mentioned that, as a tradeoff between disgregation and cohesion, fragmentation amounts to an aggregation process (Section 2), a fact we will further emphasize in Section 9. It is both surprising, and intriguing to realize that this phenomenology also occurs at the molecular scale.

In the views of Lavoisier, the founder of Chemistry as a discipline (Lavoisier 1789), there is an essential difference between mechanical and chemical forces (Tome II, Ch. V), and there is no way that any kind of Trituration, Porphirisation 8 Pulverisation (Tome II, Ch. IV) could alter the chemical nature of a constituent. This is not true; it is however only in the course of the 19th century that it was realized, mostly in a piecemeal fashion, by chance, that the effect of a mechanical action like grinding and milling ('dry' chemistry in the words of Faraday, meaning solvent-free) could produce a chemical transformation. Takacs (2013) gives an extensive historical account of what is now known as Mechanochemistry: new chemical compounds are formed when constituents in gas phase are sealed in an agitated box where solid balls collide repetitively. Do \& Friscic (2017) and Howard et al. (2018) explain the required technology (very rudimentary mixer mills, oscillating vessels enclosing grinding balls, see also the method of Kroto et al. (1985)), the type of reactions concerned, and show how this method is sometimes advantageous compared to the standard one (consisting in heating a stationary vessel), because it is faster, and less energy consuming.

What these authors do not explain however, is the principle of the method, which is still unknown and, to some extent, controversial. Tenants of the orthodox chemistry advocate the 'hot spot' theory which says that Arrhenius activation (the rate of reaction is usually proportional to $e^{-U / k_{\mathrm{B}} T}$ ) is solely responsible for chemical conversion, and that high temperatures $T$ are reached at the collision points between the balls (which is true), favoring the reaction there; that phenomenon has indeed been known for a long time with shock waves (Vielle 1900), or imploding bubbles (sonochemistry). Others claim that mechanochemistry is unique in that mechanical action is capable of effecting chemical changes that are significantly different from the familiar thermochemical reactions. Triboelectricity, plasma formation, light emission from the cracks at the surface of the colliding balls are evoked as primary causes (see Takacs (2013)).

While many causes may contribute to the same effect, it is certainly an exciting endeavor to decipher which is dominant in this fascinating problem. A collision between spherical balls generates an outward radial expulsion flow from the impact point and it is maybe not completely coincidental that the complex structures formed by this process are usually planar, with an ortho-radial symmetry, as if they had been formed on a plane, the one perpendicular to the collision direction (see e.g. Grätz et al. $(2018 a, b)$ and Fig. 15); this might be fortuitous.

\section{Jets, threads, smooth ligaments}

Jets are collimated streams of cohesive matter, usually flowing from a circular nozzle (when formed from a slit, one refers to a sheet, or planar jet, from a circular aperture, one refers to a coaxial jet etc...). In the simplest case, a jet is thus a cylinder continuously extruded from an orifice. As such, it is the best approximation to the columnar structure Plateau (1849) has shown to be unstable, precisely because it is cohesive (see Appendix A.5). Jets breakup spontaneously; they have been, for this reason since the dawn of 
science, the laboratory of many ideas, brilliant investigations, notorious mistakes, and refined analysis. Their physics has been reviewed elsewhere (see Eggers \& Villermaux (2008), including the historical perspective) and we recall here the essential elements of the fragmentation of a columnar jet with (smooth) radius $h_{0}$ :

(i) Long varicose undulations with amplitude $\epsilon$ imposed to the jet cross-section lower its average radius $\langle h\rangle=h_{0}\left(1-\epsilon^{2}\right)$ and consequently its surface energy; the jet is therefore unstable (Plateau 1849).

(ii) The driving force of the instability is Laplace pressure $\sigma / h$ from the jet curvature $h^{-1}$, sub-dominantly counter-balanced by the axial curvature $h^{\prime \prime}$ for undulations longer than $h_{0}$.

(iii) Most of the instability period is spent at moving the liquid along the jet from constricted sections to nearby thicker sections. When capillarity balances inertia, we have $\rho h_{0}^{3} \times h_{0} / t_{c}^{2} \sim \sigma / h_{0} \times h_{0}^{2}$, that is (Lord Rayleigh 1878)

$$
t_{c}=\sqrt{\frac{\rho h_{0}^{3}}{\sigma}}
$$

and when viscous stresses are the dominant resistive forces, we have $\eta / h_{0} \times h_{0} / t_{v} \sim \sigma / h_{0}$, so that (Lord Rayleigh 1892)

$$
t_{v}=\frac{\eta h_{0}}{\sigma}
$$

(iv) A cohesive column does disconnect under the sole action of surface tension forces, meaning that its radius goes to exactly zero in a finite time (as opposed to a relaxation towards zero which would require the intervention of an extra mechanism to explain disruption). An ultimate, universal regime, where the constricted radius $h$ is the only lengthscale, describes the singularity as $h \rightarrow 0$ (Eggers 1993; Day et al. 1998). For instance,

$$
\begin{aligned}
& \ddot{h} \sim-\sigma / h^{2}, \quad \text { leading to } \quad h \sim\left(t_{c}-t\right)^{2 / 3} \quad \text { in the inviscid limit, } \\
& \dot{h} \sim-\sigma / \eta, \quad \text { leading to } \quad h \sim\left(t_{v}-t\right)^{1} \quad \text { in the viscous limit. }
\end{aligned}
$$

The celebrated exponent $2 / 3$ reflects capillarity driven inertial motions (Keller \& Miksis 1983; Peregrine et al. 1990); there are other exponents in situations with additional ingredients (see Eggers \& Villermaux (2008)). The duration of this final regime represents typically a small fraction of the overall instability period (Chen \& Steen 1997; Robinson \& Steen 2001). With non-newtonian liquids like polymers (Amarouchene et al. 2001), or with dense suspensions like slurries (Chateau \& Lhuissier 2019) these universal scalings precede however other ultimate thinning laws which are specific to each system.

(v) In all cases, the fragments products of a smooth uniform steady jet have an average diameter given by the column radius $\langle d\rangle \sim h_{0}$, with possible smaller satellites (see Section 10). Strongly corrugated ligaments, through a comparatively longer dynamics preceding the short-termed final singularity, give rise to a broader continuous distribution examined in Section 9.

\subsection{Production of jets}

\subsubsection{Steady injection}

A standard jet is produced by extruding a liquid at constant flowrate $q=\pi h_{0}^{2} v_{0}$ through a circular orifice of radius $h_{0}$ at velocity $v_{0}$ (Non-circular jets present peristaltic pulsations studied by Lord Rayleigh (1879) and others, see Section 6 in Eggers \& Villermaux (2008) for a historical perspective). The very existence of a jet assumes than it has not fragmented before having formed, in other words, that the breakup 


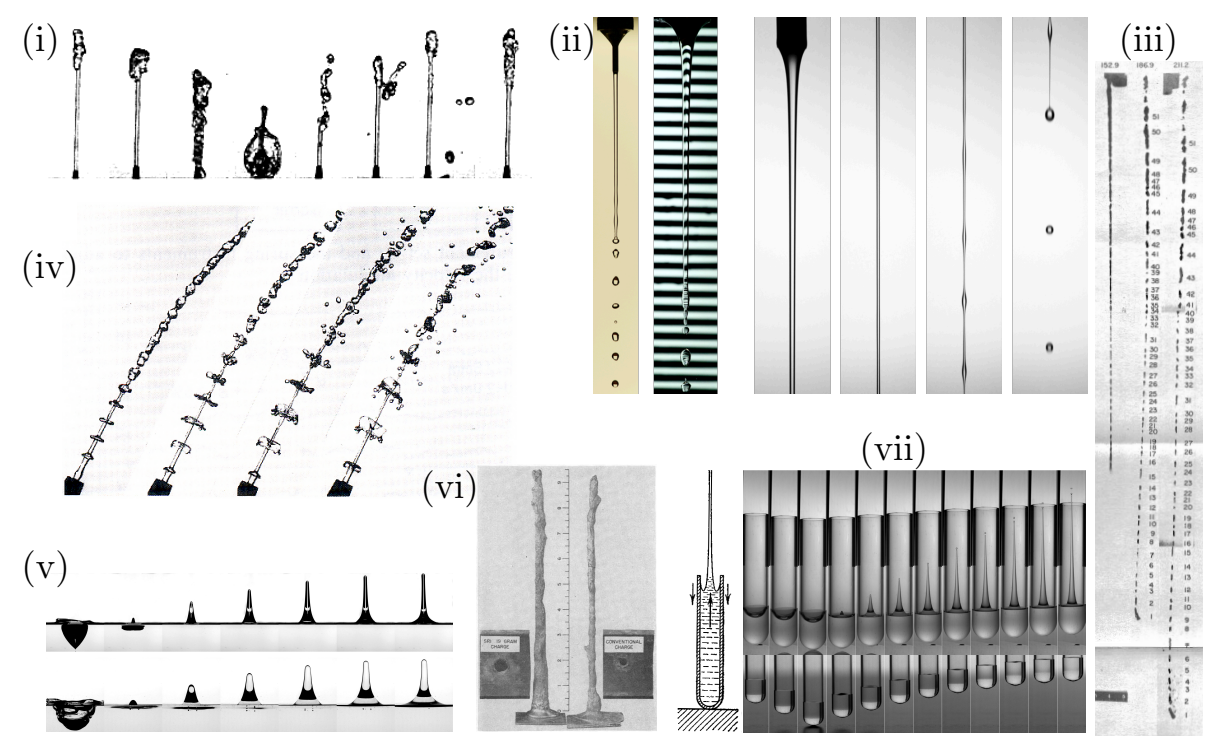

FIgURE 14. Jets. (i) Ascending jet of water falling back on itself under its own weight in a pulsating motion typical of fountains (Villermaux 1994). (ii) Several falling jets, including a fast capillary jet keeping its radius constant at breakup, and jets stretching by gravity (Le Dizès \& Villermaux 2017). (iii) The development of a jet formed by the explosion of a charge on a conical shape liner (DiPersio et al. 1960). (iv) Pulsed jets featuring shocks (Meier et al. 1992). (v) Jets eruption from the collapse of cavities at the surface of a pool (Ghabache et al. 2014b). (vi) A mold of the thin long cavity made by the trajectory of a shaped charge jet transpiercing a solid (Poulter \& Caldwell 1957). (vii) 'Pokrovski' jet formed by the impulsive acceleration of a curved liquid surface (Antkowiak et al. 2007) and a sketch by Lavrentiev \& Chabat (1980).

time $t_{c}$, say, is longer than the transit time $2 h_{0} / v_{0}$ of the fluid particles over at least one jet diameter from the nozzle exit: The critical Weber number $\rho v_{0}^{2} h_{0} / \sigma$ of the jetting/dripping transition is about 4 (Le Dizès 1997), with gravity corrections (Clanet \& Lasheras 1999). The resulting column in uniform translation remains as such (up to its capillary destabilization) as long as it does not interact with its environment, nor with external fields like gravity for instance. If gravity $\mathbf{g}=-g \mathbf{e}_{\mathbf{z}}$ is aligned with the direction of the ejection velocity $\mathbf{v}_{\mathbf{0}}= \pm v_{0} \mathbf{e}_{\mathbf{z}}$, then the jet velocity slows down when $\mathbf{v}_{\mathbf{0}}$ is oriented upwards (the jet falls back on itself in a pulsating motion typical of fountains, see Villermaux (1994)), and accelerates when it is oriented downwards (the jet stretches) since

$$
v \partial_{z} v= \pm g
$$

The jet radius remains essentially unaffected for distances from the orifice of the order of $v_{0}^{2} / g$, and thins (with $v_{0}$ downwards) for larger distances, by conservation of the flowrate $q$ as

$$
\begin{aligned}
h(z) & =\sqrt{\frac{q}{\pi v(z)}} \\
\text { with } \quad v(z) & =\sqrt{v_{0}^{2}+2 g z}
\end{aligned}
$$

Because the jet gets thinner and slenderer as it accelerates, viscous stresses become soon negligible (for $z$ larger than $\sqrt{\nu v_{0} / g}$ ), as well as curvature effects, and is therefore well described by the inertial dynamics in (8.5).

When the direction of the ejection velocity is at an angle with respect to $\mathrm{g}$, the jet 
trajectory forms a parabola as long as shear with the environment does not alter it. This alteration results in a slowing down of the fluid particles which happens sooner when the jet has fragmented into droplets, either by its own capillary instability, or because the Kelvin-Helmholtz instability has peeled-off its surface (see Section 6). The spreading jet from the fire hoses of firefighters and the Geneva lake jet are notorious examples of jets trajectories influenced by ambient air drag. For the same reason, jets of very small particles like spores are arrested at a finite distance from their ejection location by viscous air drag, describing, instead of a parabola, the well know 'sporabola' (Buller 1909-1950; Noblin et al. 2009). There was besides, at some point, a competition to make the 'fastest' jet (Yafetto 2008; Tagawa 2012; Avila et al. 2015); it is true that reaching high velocities, and therefore high jet momentum is mandatory for applications to needle-free micro-jet injection ( $\mathrm{Cu}$ et al. 2019), water jet cutting, or perforation (Poulter \& Caldwell 1957).

Viscous stresses induced by a faster coaxial stream can also be used to accelerate tiny jets for making them even thinner, a technique sometimes called 'flow focusing' (GananCalvo 1998; Ganan-Calvo \& Gordillo 2001), or 'selective withdrawal' (Cohen et al. 2001) which is widespread in microfluidics to produce mono-size droplets, or encapsulations (Utada et al. 2005).

Strong focusing is achieved by applying an electric field to conducting liquids interfaces thanks to the divergence of the electric field intensity at sharp points, competing with surface tension forces to give rise to the celebrated static 'Taylor cone' solution (see Gañán-Calvo (1997) and the review in Eggers \& Villermaux (2008)). A thin jet emanates at the tip of these cones to form all sorts of very thin threads and fibers which possibly breakup into droplets (so-called 'Electrosprays') or are used for themselves in various applications with considerable technical importance (Yarin et al. 2014).

\subsubsection{Unsteady injection}

There is a broad class of jets in nature which are not formed from a continuous source, but whose injection flowrate is time-dependent, and is often an impulse. This is in particular the case for explosions from shaped charges (Birkhoff et al. 1948; Pugh et al. 1952; Poulter \& Caldwell 1957; DiPersio et al. 1960), or in some inkjet printing technologies (Wijshoff 2010; Basaran et al. 2013). The common principle in all these situations is the violent collapse of a cavity at the surface of a liquid, concentrating the fluid impulse into a narrow region of space at the base of the cavity from which a fast jet emerges with radius $h(z, t)$ and velocity $v(z, t)$. The cavity may crunch by the collision of surface waves (Zeff et al. 2000), the capillary (Ghabache et al. 2014a), or gravitational relaxation of a hollow (Ghabache et al. 2014b), or because it is impulsively shocked (Dear et al. 1988; Antkowiak et al. 2007), among other examples.

The injection flowrate $q(t) \sim h(0, t)^{2} v(0, t)$ at the base of the jet is now time-dependent, typically decaying in time, and is a function of the jet radius at its base $h(0, t) \equiv h_{0}$ and of the injection velocity $v(0, t) \equiv v_{0}$, these two quantities having, in general, no reason to be linked to each other.

The determination of the jet shape $h(z, t)$ from a given set of initial conditions is still an open problem. Arnaud Antkowiak (Antkowiak 2013) has made a promising remark that may help to find a path to the solution. The reasoning is as follows: a particle injected at the base of the jet at time $\tau$ with velocity $v_{0}(\tau)$ will be, under a purely ballistic motion along the jet, at distance $z$ at time $t$ such that

$$
\begin{aligned}
z & =(t-\tau) v_{0}(\tau) \\
\text { or } \quad t & =\tau+\frac{z}{v_{0}(\tau)}
\end{aligned}
$$


At a given location $z$, the temporal variation of the velocity $v(z, t)=v_{0}(\tau)$ with $t$ and $\tau$ linked by (8.9) is thus

$$
\left.\frac{\partial v}{\partial t}\right|_{z}=\frac{\dot{v_{0}}(\tau)}{1-z \frac{\dot{v}_{0}(\tau)}{v_{0}^{2}(\tau)}}
$$

where $\dot{v}_{0}(\tau)=\partial_{\tau} v_{0}(\tau)$.

The interest of the above relation is that it demonstrates the consequences of the decay rate of the injection velocity $v_{0}(\tau)$ on the resulting jet's fate. Let, for instance, $v_{0}(\tau) \sim \tau^{-\beta}$ where $\beta$ is a positive exponent (we are generically interested in situations where the injection flowrate $q(t)$ decays in time because of the base injection velocity decay). We have two régimes:

(i) Either the decay of $v_{0}(\tau)$ is fast, with $\beta>1$ and in that case we have asymptotically $t \propto z / v_{0}(\tau)$ and $\partial_{t} v \propto-v_{0}^{2}(\tau) / z$ so that

$$
v(z, t) \sim \frac{z}{t}
$$

a self-similar form trivially expressing ballistic motion $\left(\partial_{t} v+v \partial_{z} v=0\right)$ found in several instances involving impacts (Villermaux \& Bossa 2011; Ghabache et al. 2014b; Vernay et al. 2015b).

(ii) Or, the decay of $v_{0}(\tau)$ is slow, with $\beta<1$ and in that case $t \propto \tau$ and $\partial_{t} v \propto \dot{v}_{0}(\tau)$ so that

$$
v(z, t) \sim v_{0}(t)
$$

meaning that there is, at large time, a memory of the injection condition in the jet which adapts adiabatically to $v_{0}(t)$ at any downstream location, in a succession of quasi steady-states.

This elegant result does not solve alone, however, for the the jet shape $h(z, t)$ which is, along the same one-dimensional description, ruled by mass conservation as

$$
\partial_{t} h+v \partial_{z} h=-\frac{1}{2} h \partial_{z} v
$$

In the adiabatic régime in (8.12), one has simply $h(z, t)=h_{0}(t)$, which requires the knowledge of the radius at its base, not a-priori given, and which should be interpreted case-by-case from additional ingredients. In the self-similar régime of (8.11), looking for solutions in the form of monomials like $h(z, t) \sim z^{a} t^{b}$ fulfilling (8.13), we have $a+b=$ $-1 / 2$, but again, nothing constrains the form of the jet (i.e. the exponent $a$ ) from the above local conservation principles, even if its shape, and dynamics (the exponent $b$ ) are linked (Ghabache et al. 2014b). Equation (8.13) can be solved (see e.g. Gekle \& Gordillo (2010)) along the same lines as for (8.10) to provide

$$
h(z, t)=\frac{h_{0}(t)}{\sqrt{1+z \frac{\partial_{z} v_{0}(\tau)}{v_{0}(\tau)}}}
$$

where $h_{0}(t) \equiv h(0, t)$ and $\left.\partial_{z} v_{0}(\tau) \equiv \partial_{z} v(z, \tau)\right|_{z=0}=-\dot{v}_{0}(\tau) / v_{0}(\tau)$, making it clear that even if the stretching rate at the base of the jet $\left.\partial_{z} v(z, \tau)\right|_{z=0}$ is known once a form of the injection velocity $v_{0}(\tau)$ has been conjectured, the unknown base jet radius $h_{0}(t)$ leaves the jet radius $h(z, t)$ undetermined.

Additional principles, or conservation laws, are needed to close the problem and understand the empirical fact that fast impulsive jets ultimately look like needles with a uniform radius shrinking in time, independent of the precise mechanism which has generated them. 


\subsection{Mode selection on a varying substate}

It is clear from the examples discussed above that the capillary stability of smooth jets and threads has to be, in general, considered on a moving substrate. This means that the thinning of the jet radius, in time, space or both has to be accounted for in the instability analysis. This seemingly simple question is in fact not so easy to answer quantitatively. Consider as a canonical example a liquid thread, falling from a nozzle by it own weight under the action of gravity. As it falls, the thread eventually fragments into drops, a fact that we understand because it has locally a columnar shape, and thus suffers a capillary instability (Appendix A.5). How far from the nozzle exit does breakup occur? Even a distracted look at the possible scenarii lets one glimpse the potential difficulties of a precise analysis:

A distance $z$ is the product of a velocity $v$ by a time $t$ (see (8.9))

$$
z=v t
$$

Capillary breakup occurs within a residence time along the jet which depends on the thread radius $h$ and on the physical properties of the liquid. This time is either the capillary time $t_{c}=\sqrt{\rho h^{3} / \gamma}$ when inertia and surface tension are solely at play, or the viscous capillary time $t_{v}=\eta h / \gamma$ if viscous effects dominantly slow-down the unstable dynamics. We also know that most of the time for breakup is spent at developing the instability about the quasi-columnar shape of the thread, the subsequent phenomena occurring around the pinching instant at the drops separation being comparatively much faster. When the jet issues from the nozzle ballistically, keeping its velocity $v_{0}$ and radius $h_{0}$ constant, the problem is indeed simple, and amounts to estimate the relevant timescale to compute the so-called 'Liquid intact length' of the jet. For instance, this length is $L=v_{0} t_{c}$ in the inertial limit (see Eggers \& Villermaux (2008) for other cases), giving

$$
\frac{L}{h_{0}} \sim \sqrt{W e}, \quad \text { with } \quad W e=\frac{\rho v_{0}^{2} h_{0}}{\sigma}
$$

Subtleties arise when the axial velocity of the jet depends on axial distance $z$. A jet falling in the direction of gravity accelerates. If fed at a constant flow rate at the nozzle, stationarity implies that the thread radius thins with increasing distances from the exit (see (8.6)). Therefore, if both $v$ and $h$ depend on downstream distance, which estimates will correctly represent the breakup distance $L$ ? Those at the nozzle exit, those at the breakup distance, or a mixture of the two? As the radius thins, the instability may switch from an inertia to a viscous dominated régime (the Ohnesorge number $O h=t_{v} / t_{c}$ increases). Then, which timescale, $t_{c}$ or $t_{v}$ should be considered to compute $L$ ?

The detailed problem is even more subtle: The capillary instability amplifies preferentially a varicose perturbation (Appendix A.5). The most amplified wavelength is proportional to the local radius $h$, the other wavelengths having a weaker growth rate. Since the jet stretches, mass conservation also implies that the distance between two adjacent instability crests increases with distance from the nozzle exit, thus weakening the growth rate of a mode initially close to maximal amplification. Concomitantly, the most amplified mode shifts towards smaller wavelengths because the jet radius diminishes, also contributing to amplification damping. The capillary instability has thus to compete with another phenomenon, namely jet stretching, characterized by another timescale $\left(\partial_{z} v\right)^{-1}$.

There are thus in-fine three timescales potentially influencing the instability time: $t_{c}$, $t_{v}$ and $\left(\partial_{z} v\right)^{-1}$ which all depend intrinsically on the distance to the nozzle $z$. Deciding a-priori which one will dominate and how is a hazardous exercise.

Deciphering the relative importance of the coupled effects mentioned above requires an instability analysis accounting for both the substrate deformation (jet stretching), and 
for the modification of the local instability dispersion relation as the jet thins (to describe the growing relative influence of viscosity). That question has been first envisaged in the very viscous limit by Tomotika (1936) using an original Lagrangian formulation. The particular case where $v$ increases linearly with $z$ was considered by Frankel \& Weihs (1985, 1987), and by Clarke (1969), Senchenko \& Bohr (2005), Sauter \& Buggish (2005), Javadi et al. (2013) and Le Dizès \& Villermaux (2017) for a gravitationally accelerated jet.

The method consists in computing, by a WKBJ technique, the amplitude gain a perturbation can reach at a given location as it is transported along the jet, and look for the wavelength for which amplification is maximum. These detailed calculations answer our questions above:

Breakup occurs when the capillary instability growth rate overcomes the stretching rate of the jet at the same location.

This is the operational criterion for reaching the appropriate scaling laws of breakup lengths $L$ :

(i) When the jet, fed at constant flowrate $q$ is initially viscous $(O h>1)$, the local stretching rate is given by $\partial_{z} v(z) \sim \sqrt{g /(2 z)}$ (see (8.7)) while the viscous capillary growth rate based on the current radius is of order $\sigma /(\eta h)=\gamma(2 g z)^{1 / 4} /(\eta \sqrt{q})$. The latter overcomes the former at a distance $L$ of order

$$
L \sim\left(q^{2} g\right)^{1 / 3}\left(\frac{\eta}{\sigma}\right)^{4 / 3}
$$

first obtained by Javadi et al. (2013), fitting approximately well their experiments.

(ii) For an initially non-viscous jet $(O h \ll 1)$, the local jet stretching rate is still $\partial_{z} v(z) \sim \sqrt{g /(2 z)}$ while the inviscid capillary growth rate based on the current radius is now of order $\sqrt{\sigma / \rho h^{3}}=\sqrt{\sigma / \rho}(2 g z)^{3 / 8} / q^{3 / 4}$. The latter overcomes the former at a distance $L$ of order

$$
L \sim\left(q^{6} g\right)^{1 / 7}\left(\frac{\rho}{\sigma}\right)^{4 / 7}
$$

as inferred by Le Dizès \& Villermaux (2017) who have also discussed how these scalings are affected by the way the initial perturbation noise is introduced, either at the orifice exit (localized perturbation), or all along the jet (ambient noise). Mean droplets sizes follow from this analysis.

Let us finally mention that the above breakup criterion had been anticipated by Tjahjadi \& Ottino (1991) in the formation of emulsions by stirring and by Villermaux (2012) in the context of the filamentation of molten silicates like clinker (a mixture of limestone and clay used in the production of cement), angel hair, glass wool, or Pele's hair (named after Pele, the Hawaiian goddess of volcanoes), those long and thin solid fiber formed during the eruptions of volcanoes, at the flank of which they are found. These object are so violently stretched during eruptions that they thin down to fractions of a millimeter, and solidify before having broken up.

\section{The post Plateau-Rayleigh era: Corrugated ligaments}

By 'post' we refer to the works which, after Savart (1833a) had first documented the phenomenon, Plateau (1849) had understood the origin of the instability, Lord Rayleigh (1878) had computed its growth rate and Weber (1931) had given a long wave description of it, have concentrated on the physics of the ultimate, singular breakup of a liquid thread into disjointed droplets on one side, and on the other side those which have 
addressed the inherent multiplicity of droplets sizes. The modern developments on the first aspect are reviewed in Eggers (1997), and we concentrate here on the second, namely the fragmentation products per-se.

\subsection{Paradigm of the corrugated ligament}

The smooth, infinitely long columnar structure with uniform cross-section is an idealization of nature. Even an approximation of such a long uniform cylinder is actually difficult to produce experimentally; Plateau (1849) used soap films to minimize the role of gravity but a fast jet emanating from a nozzle smaller than the capillary lengthscale is a safer means. However in nature, drops commonly form from the breakup of ligaments which have a finite length, and are corrugated with an uneven cross-section. Since they represent the last but one step before the formation of drops, understanding their dynamics is paramount to understand the ubiquitous diversity of drops sizes in sprays.

Besides their tendency to give rise to the standard capillary instability, corrugations have an intrinsic dynamics, and are continuously produced on a finite-size ligament: on an initially rough ligament, small sections feed larger adjacent sections but as they shrink in size, their associated growth rate changes. Also, the Taylor-Culick recession of the ligament ends feeds the core of the ligament with capillary waves (Schulkes 1996; Clasen et al. 2009; Hoepffner \& Paré 2013). These waves are dispersive (see e.g. Duchemin et al. (2015)) and travel long distances when their wavelength is smaller than the ligament (mean) radius because they are stable, with a non-zero group velocity (see Appendix A.4). These waves possibly overlap with other preexisting waves (Driessen et al. 2014; Doméjean et al. 2016), thus altering dynamically the corrugations landscape of the ligament, and broadening its spectrum (Stone \& Leal 1989), a phenomenon also known for gravity waves (Falcon et al. 2007; Redor et al. 2019). Corrugations may also be due to internal motions within the ligament, as these remnant from the liquid bulk from which the ligament has been stripped-off either because the bulk is already turbulent (Goodridge et al. 1996), or as a result of the instability causing the stripping, as a shear for instance. Being constantly 'hesitant' between local amplification, and longitudinal propagation (Villermaux 2009), the capillary instability thus develops on a 'noisy' substrate, a fact known to broaden the drops sizes distribution after breakup (see Zhao et al. (2019) for thermally activated noise).

The result of all these contributions is a landscape of random corrugations which, because they have diverse origins, are essentially uncorrelated with each other. In particular, the correlation length along a ligament does not exceed its local radius. This legitimates the caricature of a ligament as made of a collection of adjacent blobs with random diameters $d$, each interacting with its neighbors in which it possibly empties, or on which it possibly feeds though an aggregation type of dynamics driven by capillary forces.

\subsection{Inverse cascade of aggregations: lessons from a necklace of magnets}

We have inaugurated our discussion by noticing that in fragmentation matters, one must wonder about aggregation in the first place. The magnets necklace was our starting example in Section 2.3. We have seen how the fragments are produced from an inverse cascade of aggregation of clusters, each carrying an increasing number of beads $n$ through the cascade. We now describe briefly the construction mechanism of their distribution.

In the limit of a continuous spectrum for the fragment size $n$, the rate of change of $\mathbb{b}(n, t) \mathrm{d} n$, the number of fragments having sizes between $n$ and $n+\mathrm{d} n$ at time $t$ is given 
by (Smoluchowski 1917)

$\partial_{t} \mathrm{~b}(n, t)=\frac{1}{2} \int_{0}^{\infty} K\left(n^{\prime}, n-n^{\prime}, t\right) \mathfrak{b}\left(n^{\prime}, t\right) \mathfrak{b}\left(n-n^{\prime}, t\right) \mathrm{d} n^{\prime}-\mathfrak{b}(n, t) \int_{0}^{\infty} K\left(n, n^{\prime}, t\right) \mathfrak{b}\left(n^{\prime}, t\right) \mathrm{d} n^{\prime}$

which describes how the distribution changes by the aggregation operation $n-n^{\prime}+n^{\prime} \rightarrow n$, at a rate given by the so-called Kernel $K\left(n-n^{\prime}, n^{\prime}, t\right)$. This formulation involves the cluster sizes only when the physical problem does not present strong spatial correlations and/or segregation phenomena forcing, for instance, small clusters to interact preferentially with other small clusters. On the contrary, all clusters are liable to interact with the entire population of clusters in the Smoluchovski formulation. This limit corresponds to an absence of correlation between the sizes of the aggregating fragments, that is when the correlation coefficient (the sum applies to all $i$ fragments along the necklace)

$$
C(p, t)=\frac{\sum_{i}\left(n_{i}-\langle n\rangle\right)\left(n_{i+p}-\langle n\rangle\right)}{\sum_{i}\left(n_{i}-\langle n\rangle\right)^{2}}
$$

vanishes at all times and for all neighboring clusters (i.e. for $p>1$ ), a condition indeed fulfilled in the necklace problem.

A solution to this equation is known for a limited number of kernels only and in general one relies on asymptotic techniques to study the asymptotic behavior of $\mathfrak{b}(n, t)$ at long times (Leyvraz 2003). In typical applications, such as aggregation in chemical reactors (Curl 1963) or aggregation of dust grains in the atmosphere (Friedlander 2000) or in a turbulent interstellar domain, collisions occur because particles are set to move at random by the agitation of the underlying medium (Ilievski et al. 2011), but may also move on their own driven by attractive forces (Bleibel et al. 2011).

Equation (9.1) can be written in terms of the probability density function $\mathrm{p}(n, t)=$ $\mathfrak{b}(n, t) / B(t)$ where $B(t)=\int_{0}^{\infty} \mathfrak{b}(n, t) \mathrm{d} n$ is the total number of fragments. The equation of evolution of $B(t)$ can be deduced from (9.1),

$$
\partial_{t} B(t)=\frac{1}{2} \int_{0}^{\infty} \mathfrak{b}(n, t) \int_{0}^{\infty} K\left(n, n^{\prime}, t\right) \mathfrak{b}\left(n^{\prime}, t\right) \mathrm{d} n^{\prime} \mathrm{d} n
$$

Introducing the rate of aggregation

$$
r\left(n, n^{\prime}, t\right)=\frac{1}{2} K\left(n, n^{\prime}, t\right) B(t)
$$

we obtain for $\mathrm{p}(n, t)$

$$
\begin{aligned}
\partial_{t} \mathfrak{p}(n, t)= & \mathbb{p}(n, t)\left\{\int_{0}^{\infty} \int_{0}^{\infty} r\left(m, n^{\prime}, t\right) \mathfrak{p}(m, t) \mathfrak{p}\left(n^{\prime}, t\right) \mathrm{d} n^{\prime} \mathrm{d} m-2 \int_{0}^{\infty} r\left(n, n^{\prime}, t\right) \mathfrak{p}\left(n^{\prime}, t\right) \mathrm{d} n^{\prime}\right\} \\
& +\int_{0}^{\infty} r\left(n-n^{\prime}, n^{\prime}, t\right) \mathfrak{p}\left(n-n^{\prime}, t\right) \mathfrak{p}\left(n^{\prime}, t\right) \mathrm{d} n^{\prime}
\end{aligned}
$$

which does not look more engaging than the original formulation in (9.1) except that, in this problem like in others (Villermaux \& Almarcha 2016), the rate of aggregation decreases with the fragment sizes: small fragments are lighter and reconnect at a much faster pace than massive fragments. We thus make the following caricature: large fragments are massive and thus only contribute to the global dynamics by attracting small fragments while small fragments are likely to aggregate to any other fragment in the current distribution. Large/small depends on the fragments size $n$ compared to a threshold $n_{\star}$, to be determined by an extra criterion, a function of the details of the interaction between the clusters. In this idealization, aggregation up to $n_{\star}$ complies with 
Smoluchowski's dynamics at a constant rate $r$ while it is zero among sizes larger than $n_{\star}$. Thus (9.5) reduces to

$$
\partial_{t} \mathbb{P}(n, t)=-r \mathbb{P}(n, t) \int_{0}^{n_{\star}} \mathbb{P}\left(n^{\prime}, t\right) \mathrm{d} n^{\prime}+r \int_{0}^{n_{\star}} \mathbb{P}\left(n-n^{\prime}, t\right) \mathbb{P}\left(n^{\prime}, t\right) \mathrm{d} n^{\prime}
$$

which expresses the partial convolution of $\mathfrak{p}\left(n^{\prime}, t\right)$ with itself. For $n_{\star} \rightarrow \infty$ (all sizes aggregate at a constant rate $r$ ) we recover the classical kinetic aggregation equation whose solution is an exponential distribution $\mathrm{p}(n)=e^{-n /\langle n\rangle} /\langle n\rangle$ with $\langle n\rangle \sim e^{r t}$ (Smoluchowski 1917; Leyvraz 2003). Introducing the Laplace transform $p(s, t)$ of $\mathfrak{p}(n, t)$

$$
p(s, t)=\int_{0}^{\infty} \mathrm{e}^{-n s} \mathrm{p}(n, t) \mathrm{d} n
$$

we have from (9.6)

$$
\partial_{t} p=r p\left(-\int_{0}^{n_{\star}} \mathfrak{p}(n, t) \mathrm{d} n+\int_{0}^{n_{\star}} \mathrm{e}^{-n s} \mathfrak{p}(n, t) \mathrm{d} n\right)
$$

This equation has no analytical solution but is, when $n_{\star}$ is finite as first noticed by Villermaux \& Duplat (2003) in the context of scalar mixing, tangent to

$$
\partial_{t} p=r^{\prime}\left(-p+p^{1+1 / \nu}\right)
$$

with $\nu$ and $r^{\prime}$ yet unknown, both being a function of $n_{\star}$ and $r$. The distribution of the cluster sizes relative to their mean $x=n /\langle n\rangle$ such that $\mathfrak{p}(n, t)=\mathbb{E}(x) /\langle n\rangle$ tends asymptotically towards a Gamma distribution of order $\nu$

$$
\mathbb{E}(x)=\frac{\nu^{\nu}}{\Gamma(\nu)} x^{\nu-1} e^{-\nu x}
$$

which constitutes the first important result of this partial convolution theory since it provides the shape of the clusters distribution.

In order to compute the -still unknown- parameter $\nu$, we further expand $e^{-s n} \approx$ $1-n s+n^{2} s^{2} / 2+O\left(s^{3}\right)$ and write $(9.8)$ as

$$
\partial_{t} p=r\left(-\left\langle n_{p}\right\rangle s+\left(\frac{\left\langle n_{p}^{2}\right\rangle}{2}+\langle n\rangle\left\langle n_{p}\right\rangle\right) s^{2}\right)+O\left(s^{3}\right)
$$

with

$$
\left\langle n_{p}\right\rangle=\int_{0}^{n_{\star}} n \mathrm{p}(n, t) \mathrm{d} n, \quad\left\langle n_{p}^{2}\right\rangle=\int_{0}^{n_{\star}} n^{2} \mathrm{p}(n, t) \mathrm{d} n
$$

Since $p(s, t)$ is also the generating function of the moments of $p(n, t)$, that is $p(s, t)=$ $1-\langle n\rangle s+\left\langle n^{2}\right\rangle s^{2} / 2+O\left(s^{3}\right)$, term by term identification in Eqs. (9.9) and (9.11) yields at order $s$

$$
\frac{r^{\prime}}{r}=\nu \frac{\left\langle n_{p}\right\rangle}{\langle n\rangle}
$$

and at order $s^{2}$, using the previous result,

$$
\frac{\left\langle n_{p}\right\rangle}{\langle n\rangle}\left(\frac{\left\langle n^{2}\right\rangle}{\langle n\rangle^{2}}+\frac{1-\nu}{\nu}\right)=\frac{\left\langle n_{p}^{2}\right\rangle}{\langle n\rangle^{2}}
$$

so that, in the scaled units of $\mathbb{E}(x)$, we have

$$
\left\langle x^{2}\right\rangle+\frac{1}{\nu}-1=\frac{\left\langle x_{p}^{2}\right\rangle}{\left\langle x_{p}\right\rangle},
$$


with

$$
\left\langle x_{p}\right\rangle=\int_{0}^{x_{\star}} x \mathbb{E}(x) \mathrm{d} x, \quad\left\langle x_{p}^{2}\right\rangle=\int_{0}^{x_{\star}} x^{2} \mathbb{E}(x) \mathrm{d} x
$$

Since for $\mathbb{E}(x)$ given by the Gamma distribution in (9.10) we have $\langle x\rangle=1$ by construction and $\left\langle x^{2}\right\rangle=1+1 / \nu$, we obtain finally

$$
\nu=2 \frac{\int_{0}^{x_{\star}} x f(x) \mathrm{d} x}{\int_{0}^{x_{\star}} x^{2} f(x) \mathrm{d} x} \approx \frac{2}{x_{\star}}
$$

which is the second important result of this partial convolution theory since it relates the width of the distribution (the Gamma order $\nu$ ) with a dynamical parameter of the physical system, namely the cut-off size for aggregation relative to the mean cluster size $x_{\star}=n_{\star} /\langle n\rangle$ (the approximate value $\nu \approx 2 / x_{\star}$ is all the more valid that $x_{\star}$ is large).

The last step consists in linking the critical size $n_{\star}$ to the microscopic dynamics of the interacting beads. Vledouts et al. (2015) have shown that the aggregation time between two clusters of size $p$ and $q$ (see Section 2.3) is

$$
t_{p, q} \approx \frac{a}{u_{p, q}}\left(\epsilon_{p, q}-1\right)^{-5 / 6}
$$

A fragment of size $n$ will aggregate with another of size $2\langle n\rangle-n$ to form a fragment of size $2\langle n\rangle$ if the typical aggregation time for these two fragments is less than the aggregation time between two typical fragments of the distribution, say of sizes $\langle n\rangle \pm$ $\langle n\rangle / \sqrt{\nu}$, with $\langle n\rangle / \sqrt{\nu}$ standing for the standard deviation of the cluster size population. This criterion reads

$$
\frac{t_{n, 2\langle n\rangle-n}}{t_{\langle n\rangle+\langle n\rangle / \sqrt{\nu},\langle n\rangle-\langle n\rangle / \sqrt{\nu}}} \lesssim \mathcal{O}(1)
$$

which provides from (9.18) with $x_{\star}=n_{\star} /\langle n\rangle$

$$
x_{\star}=1 / 4 \text { and } \nu=8,
$$

fitting well the observed Gamma distribution in the $n /\langle n\rangle$ units. The rate of increase of the mean itself is given by

$$
\frac{\mathrm{d}\langle n\rangle}{\mathrm{d} t}=\frac{\langle n\rangle}{t_{\langle n\rangle,\langle n\rangle}}
$$

which saturates when $\epsilon_{\langle n\rangle,\langle n\rangle} \rightarrow 1$ as the inverse cascade interrupts because $t_{\langle n\rangle,\langle n\rangle} \rightarrow \infty$, thus marking the end of the fragmentation process of the necklace.

The lessons to be drawn from this necklace exercise are the following:

(i) In a process where cohesion and disgregation forces compete at the initial advantage of the later, aggregation of the fragments proceeds at an ever slowing down pace up to equilibrium.

(ii) The corresponding inverse cascade is well represented by a partial convolution scenario where only fragments which aggregate sufficiently fast are allowed to recombine within the evolution time of the mean. This convolution route selects Gamma distributions.

(iii) The threshold size in units of the mean $x_{\star}$ resulting from the above timescale ratio sets the width of the distribution (i.e. the Gamma order $\nu \sim 1 / x_{\star}$ ).

\subsection{Rough ligaments: rearrangements versus instability timescales}

We consider a ligament with volume $\Omega=\pi h_{0}^{2} L$ and average radius

$$
\langle h\rangle=h_{0}\left(1-\epsilon^{2}\right)
$$


(i)

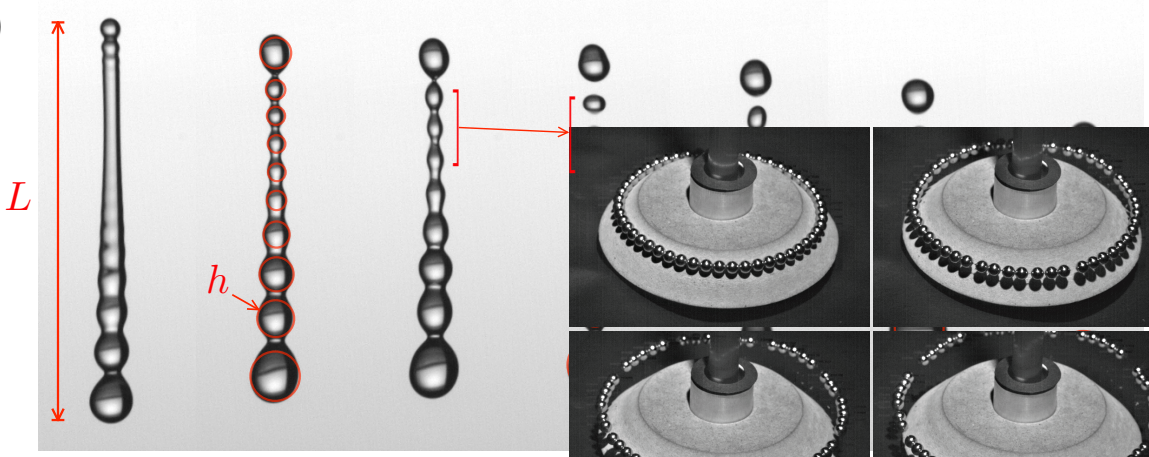

(ii)

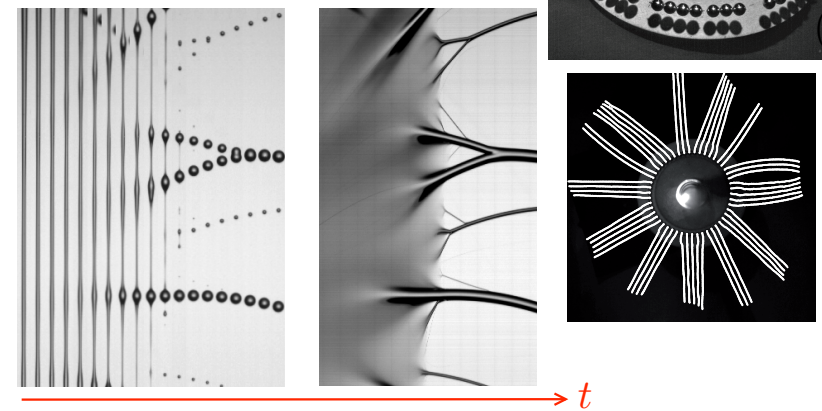

(iii)

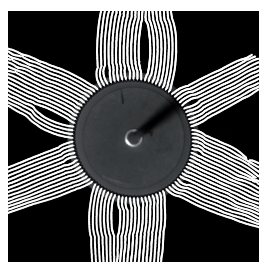

FiguRE 15. Ligament aggregation dynamics: (i) Capillary driven coarsening, and instability of an isolated water ligament; successives images are separated by about $10^{-3} \mathrm{~s}$, the initial ligament diameter is about a millimeter. Sub-portions of a ligament with uniform cross-section (marked) destabilize in uniform droplets sizes, while the other portions undergo coalescence and form bigger droplets. The net volume $\Omega$ of the volume is conserved while its length $L(\mathbb{E})$ and surface area $S(\mathbb{E})$ decay in time, as well as the number of blobs $B(\mathbb{E})$ needed to cover it (see Section 9.4). (ii) Same phenomenon as above, from a viscous thread, and its spatio-temporal trace showing the inverse cascade of aggregations between the blobs constitutive of the ligament. (iii) Illustration of the inverse cascade in the magnet necklace experiment, for two initial divergence velocities (Vledouts et al. 2015).

where $\epsilon^{2}=\left\langle(h-\langle h\rangle)^{2}\right\rangle /\langle h\rangle^{2}$ denotes the mean squared relative corrugations amplitude along the ligament. We have seen in Section 8 that such a jet destabilizes within the capillary time

$$
t_{c}=\sqrt{\frac{\rho h_{0}^{3}}{\sigma}}
$$

We want to describe the dynamics of the corrugations of the ligament, and notably the one of their amplitude. These involve longitudinal, and radial rearrangements of fluid particles from which concentrated blobs progressively built by growing or decaying in size, each separated from its neighbors by bridges conducting the longitudinal rearrangements, and whose breakup freezes the blobs size repartition, thus setting the final drops.

The rearrangements are made at constant volume, and the arrow of time of the evolution of the blobs assembly points towards the decay of its surface area, in compliance with the Plateau argument. The length of the ligament, and the number of blobs are not a-priori constrained by any principle, but empirical evidence indicates that both decay in time.

Corrugations develop on an unstable substrate, whose growth rate is maximum for longitudinal sizes of the order of the local ligament radius. Blobs have thus the size of the local radius, which itself fluctuates along the ligaments. This is confirmed by the 
observation that the correlation length of the radius fluctuations is of the order of the ligament average radius.

We thus portray the ligament as an assembly of spherical adjacent blobs, somewhat like beads on a string with the notable difference that these beads are not isolated but interact, may feed, or be fed by their environing beads. The driving force of the mass transfers between the beads is obviously the Laplace capillary pressure. With this in mind, a simple (inviscid) dynamical model for the longitudinal velocity $v(z, t)$ and ligament radius radius $h(z, t)$ is

$$
\begin{array}{r}
\rho \partial_{t} v=-\partial_{z} p \\
\partial_{t} h^{2}+\partial_{z}\left(h^{2} v\right)=0
\end{array}
$$

giving $v \approx \dot{h}$ and

$$
\ddot{h}=-\frac{1}{\rho} \frac{\Delta p}{h}
$$

once derivatives along $z$ have been coarse-grained at the correlation length $h$ (i.e. $\left.\partial_{z} \equiv 1 / h\right)$, the local radius. In this blob description, the pressure is simply inversely proportional to the local radius (namely $\sigma / h$ ) and if a blob is connected with much bigger blobs with weak internal pressure, then $\Delta p \approx \sigma / h$. The blob empties in that case according to the familiar (see Section 8 and Eggers \& Villermaux (2008) for references and many more details) finite-time singularity

$$
h \sim\langle h\rangle\left(1-t / t_{c}\right)^{2 / 3}
$$

for a blob with initial radius of order, say, $\langle h\rangle$.

This is not, however, the typical situation occurring along a ligament. A given blob is more likely connected to alike blobs, whose radii $h \pm \delta$ are affected by a random fluctuation of order $\delta$ such that $\langle\delta\rangle=0$ and $\left\langle\delta^{2}\right\rangle=\langle h\rangle^{2} \epsilon^{2}$. The value of $\delta$ is random, but is assigned positively, and negatively to the radius of each adjacent blobs, thus describing a local radius gradient along the ligament, of intensity $\delta / h$. We thus account for the adverse presence of adjacent blobs through a mean field correction involving a pressure term averaged over the positive, and negative excursions of the typical corrugations noting that $\frac{1}{2}\left[(h+\delta)^{-1}+(h-\delta)^{-1}\right]=h /\left(h^{2}-\delta^{2}\right)^{-1}$. The driving pressure writes

$$
\begin{aligned}
\Delta p & =\sigma\left(\frac{1}{h}-\frac{1}{h\left(1-\delta^{2} / h^{2}\right)}\right) \\
& =-\frac{\sigma}{h^{3}} \delta^{2}
\end{aligned}
$$

Obviously, $\Delta p=0$ for a stationary array of all identical blobs when $\delta=0$ and for $\delta \neq 0$, a longitudinal flow sets-in (Sierou \& Lister 2004; Brasz et al. 2018) which interferes with the capillary instability (Decent \& King 2008). The dynamics of a blob radius $h(t)$ in the assembly, now incorporating the influence of the adjacent blobs is thus given by

$$
\ddot{h}=\frac{\sigma}{\rho} \frac{\delta^{2}}{h^{4}}>0 .
$$

Its acceleration is positive reflecting the coarsening process through which blobs feed on their smaller adjacent neighbors. The environment of a blob with radius $h$ averaged over the blobs assembly $(\sigma / \rho)\left\langle\delta^{2} / h^{4}\right\rangle \approx(\sigma / \rho) \epsilon^{2} /\langle h\rangle^{2}$ is thus such that its average time of growth $t_{h}^{-2}=\langle\ddot{h} / h\rangle$ is given by

$$
\frac{1}{t_{h}^{2}}=\frac{1}{t_{c}^{2}} \frac{\langle h\rangle}{h} \epsilon^{2}
$$


at leading order in $\epsilon^{2}$.

Along a ligament, longitudinal rearrangements compete with the capillary instability which, as seen from (9.27) for instance, occurs within $t_{c}$. However, all blobs sizes smaller than $h_{\star}=\epsilon^{2}\langle h\rangle$ have, according to (9.31), a growth time $t_{h}$ smaller than the capillary time $t_{c}$. These blobs have thus time to coarsen, to participate to the inverse cascade, while larger sizes grow at a slower pace, and suffer the capillary instability.

In view of the partial convolution theory recalled in Section 9.2 above, the critical size $h_{\star}$ sets the extent of the convolution range: blobs sizes will grow by the addition of, at most, an increment $h_{\star}$ within the time interval $t_{c}$. If $\delta$ is distributed randomly along the ligament, the additions are made at random between adjacent blobs and their size distribution is a Gamma distribution as in (9.10) of order given by (9.17)

$$
\begin{aligned}
\nu & \sim \frac{\langle h\rangle}{h_{\star}}=\epsilon^{-2} \\
& =\frac{\langle h\rangle^{2}}{\left\langle h^{2}\right\rangle-\langle h\rangle^{2}}
\end{aligned}
$$

We comment further on this blobs representation, and on the meaning of the limit values of $\nu$ in the next Section.

\subsection{Blobs on a ligament: fractional convolutions, Gamma distributions}

The longitudinal rearrangements period in a ligament lasts for a time of the order of $t_{c}$, a time which serves as the reference timescale in what follows

$$
\mathbb{t}=\frac{t}{t_{c}}
$$

Let $\mathbb{b}(d, \mathbb{E})$ be the distribution of the number of blobs of size $d$ constitutive of a ligament at time $t$ (i.e. $\mathbb{b}(d, \mathbb{E}) \mathrm{d} d$ is the number of blobs with size between $d$ and $d+\mathrm{d} d$ ), and its Laplace transform

$$
b(s, \mathbb{E})=\int_{0}^{\infty} \mathfrak{b}(d, \mathbb{E}) e^{-s d} \mathrm{~d} d
$$

The total number of blobs, irrespective of their size $B(\mathbb{L})$, the length of the ligament (the sum $L(\mathbb{E})$ of the blobs diameters $d$ ), its surface area (the sum $S(\mathbb{E})$ of the blobs projected area $d^{2}$ ) and its volume (the sum $\Omega(\mathbb{E})$ of the blobs volumes $d^{3}$ ) are obtained from the generating function $b(s, \mathbb{E})$ as (we denote $\left.b^{\prime}(0, \mathbb{E})=\left.\partial_{s} b(s, \mathbb{E})\right|_{s=0}\right)$

$$
\begin{aligned}
B(\mathbb{E}) & =\int_{0}^{\infty} \mathfrak{b}(d, \mathbb{E}) \mathrm{d} d=b(0, \mathbb{E}) \\
L(\mathbb{E}) & =\int_{0}^{\infty} d \mathfrak{b}(d, \mathbb{E}) \mathrm{d} d=-b^{\prime}(0, \mathbb{E}) \\
S(\mathbb{E}) & =\int_{0}^{\infty} d^{2} \mathfrak{b}(d, \mathbb{E}) \mathrm{d} d=b^{\prime \prime}(0, \mathbb{E}) \\
\Omega(\mathbb{E}) & =\int_{0}^{\infty} d^{3} \mathfrak{b}(d, \mathbb{E}) \mathrm{d} d=-b^{\prime \prime \prime}(0, \mathbb{E})
\end{aligned}
$$

Corrugations, whose dynamics is driven by surface tension obey, for the reasons explained in Section 9.1, a self-convolution rule analogous to (9.9), described by (Villermaux et al. 2004)

$$
\partial_{\mathbb{t}} b=-B^{\gamma-1} b+\frac{1}{3 \gamma-2} b^{\gamma}
$$



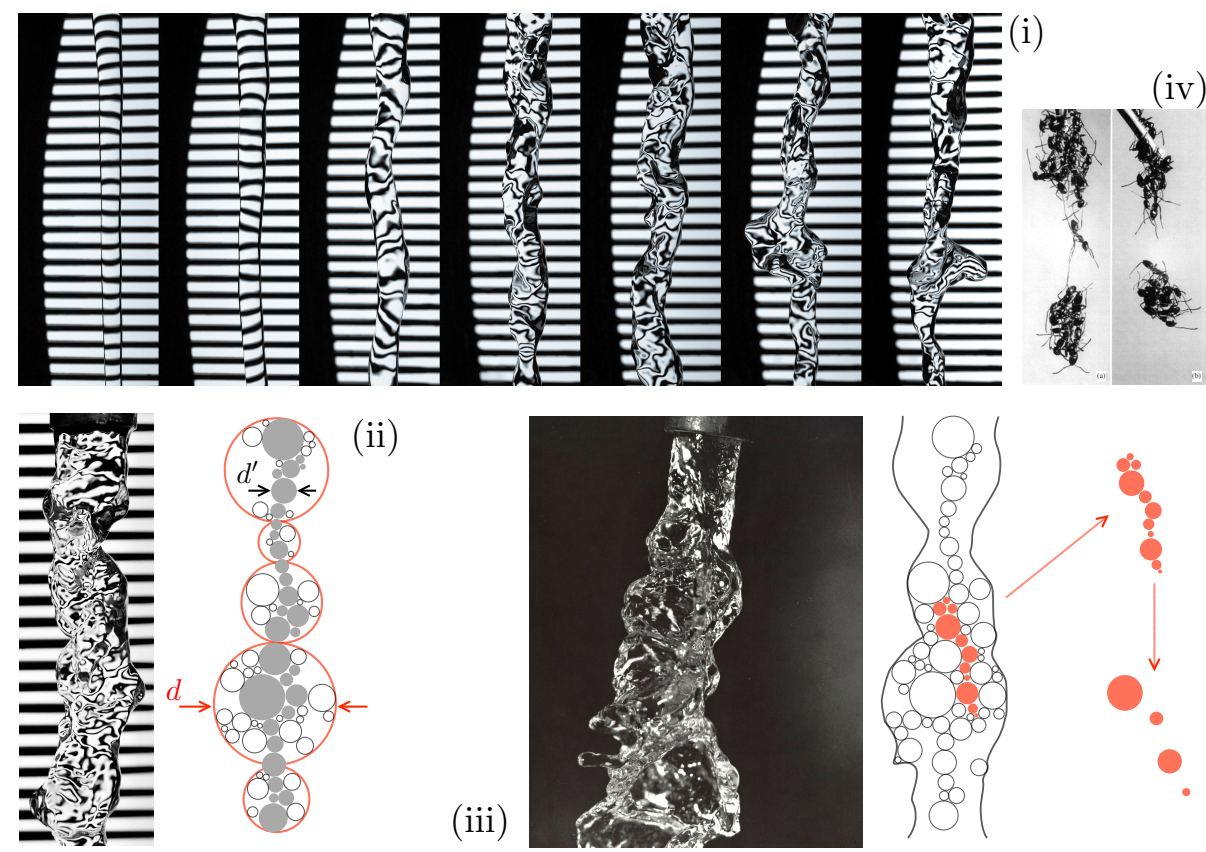

(ii)
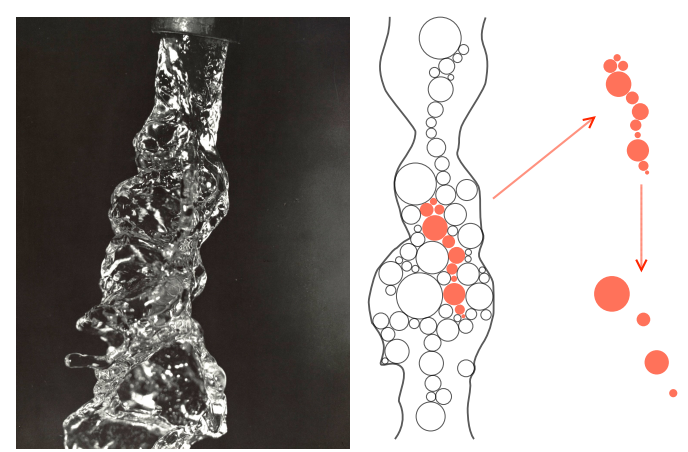

Figure 16. (i) When lit from behind through a comb grid, a water jet is hardly discerned as its smooth surface leaves the projected grid unaltered. Increasing the flowrate causes turbulent hieratic motions to set in, which distort and corrugate the jet. Gridlines caustics are more distorted for higher flowrates, revealing ever smaller surface corrugations, varying on lengthscales smaller than the jet diameter. Eventually, turbulent fluctuations are intense enough to overcome surface tension confinement, and the jet fragments. (ii) The decomposition of a ligament into blobs, themselves composed of sub-blobs. (iii) Stripping of sub-blob from a ligament, and its rupture into stable drops illustrating how liquid fragmentation results from a trade-off between disaggregation, and cohesion forces. The pictures are also a tribute to Harold E. Edgerton, whose first Moment of Vision, in 1932, was a turbulent jet from a faucet (Edgerton 1979). (iv) The dripping of a cohesive 'liquid' made of active particles (ants grabbing each other) hanging on a rod; the falling droplets are distributed in size (Thereulaz et al. 2001).

where the parameter $\gamma \gtrsim 1$ is very sensibly related to the corrugation state of the ligament. The factor $3 \gamma-2$ reflects the conservation of the ligament volume. If its surface were conserved (as for rearrangements in two-dimensions), this factor would be $2 \gamma-1$. The blobs population is left unchanged through (9.40) when $\gamma=1$.

The convolution operates on the blob size distribution $\mathfrak{b}(d, \mathbb{E})$, expressing an addition in sizes space, while to total volume $\Omega=-b^{\prime \prime \prime}(0, \mathbb{E})$ is conserved through the evolution of the size assembly $\left(\partial_{\mathbb{t}} \Omega=0\right)$; it writes

$$
\Omega=\frac{\gamma}{2}\left((\gamma-2) L^{3} B^{-2}+3 L S B^{-1}\right)
$$

so that, since $\Omega$ is independent of time, so are $L^{3} B^{-2}$ and $L S B^{-1}$, giving

$$
\begin{aligned}
& L=\left(\frac{\Omega}{\gamma(2 \gamma-1)}\right)^{1 / 3} B^{2 / 3} \\
& S=\gamma\left(\frac{\Omega}{\gamma(2 \gamma-1)}\right)^{2 / 3} B^{1 / 3}
\end{aligned}
$$


with the total number of blobs given by

$$
B(\mathbb{E})=B(0)\left(1-3 \frac{(\gamma-1)^{2}}{3 \gamma-2} \frac{\mathbb{E}}{B(0)^{1-\gamma}}\right)^{\frac{1}{1-\gamma}}
$$

Random additions between adjacent blobs coarsen the population of blobs (when $\gamma>1$ ) whose average size increases in time, at the expense of the number of blobs $B$ which decreases in time as well as the length $L$ and surface area $S$ of their support.

The decrease of the ligament surface area $S$ is compatible with the thermodynamic Plateau principle stating that an isolated system evolves so as to lower its surface energy. However, this decrease is not, in the present theory, a direct consequence of that precise principle, but rather a consequence of a principle of aggregation between sub-parts of a volume otherwise globally conserved.

The average blob size $\langle d\rangle=L / B$ and variance, with $\left\langle d^{2}\right\rangle=S / B$ are such that

$$
\begin{aligned}
\frac{\left\langle d^{2}\right\rangle-\langle d\rangle^{2}}{\langle d\rangle^{2}} & =\gamma-1 \\
& =\epsilon^{2} \quad \text { from (9.33) }
\end{aligned}
$$

at all times during the coarsening process provided $\gamma$ is itself constant in time, which happens to be an experimental fact (see Fig. 18 in Marmottant \& Villermaux (2004a)). In other words, the relative corrugation state of the ligament is preserved during the coarsening process and will therefore set the relative width of the drop size distribution $\mathbb{b}(d, \mathbb{E}=1)$ obtained at the breakup time $\mathbb{E}=1$.

The distribution of blobs sizes $\mathbb{b}(d, \mathbb{E})$ is itself a solution of the evolution (9.40). A change of variables (see e.g. Friedlander (2000))

$$
\begin{gathered}
\qquad(d, \mathbb{E})=\frac{B^{4 / 3}}{\Omega^{1 / 3}} \mathbb{E}(\eta, \tau) \\
\text { with } \quad \eta=d\left(\frac{B}{\Omega}\right)^{1 / 3}, \text { and } \tau=\left(\frac{B(\mathbb{E})}{B(0)}\right)^{2}
\end{gathered}
$$

which consists in counting blobs sizes $d$ in units of their current average $\langle d\rangle \sim(\Omega / B)^{1 / 3}$ and measuring time through the evolution of the number of blobs, links the Laplace transform $f\left(s^{\prime}, \tau\right)=\int_{0}^{\infty} \mathbb{E}(\eta, \tau) e^{-s^{\prime} \eta} \mathrm{d} \eta$ of the scaled distribution $\mathbb{E}(\eta, \tau)$ to the one of the original distribution as $b(s, \mathbb{E})=B f\left(s^{\prime}, \tau\right)$ with $s^{\prime}=(\Omega / B)^{1 / 3} s$. Though $(9.40), f\left(s^{\prime}, \tau\right)$ converges towards

$$
f\left(s^{\prime}\right)=\left(1+(\gamma-1) s^{\prime}\right)^{-\frac{1}{\gamma-1}}
$$

independent of $\tau$. Its inverse in sizes space defines $\mathbb{b}(d)$ as a Gamma distribution

$$
\mathbb{b}(d /\langle d\rangle)=B(1) \frac{\nu^{\nu}}{\Gamma(\nu)}\left(\frac{d}{\langle d\rangle}\right)^{\nu-1} e^{-\nu \frac{d}{\langle d\rangle}}
$$

of order (Villermaux et al. 2004; Villermaux 2007)

$$
\nu=\frac{1}{\gamma-1}
$$

The surface tension driven coarsening process of the ligament, accompanying its destabilization into disjointed droplets gives rise to a self-preservating blobs size distribution identical, for the same reason, to the cluster size distribution of the exploding magnets necklace. The capillary time marks the end of the inverse coalescence process between 
adjacent blobs which are, at $\mathbb{E}=1$ de-facto too distant to exchange fluid particles anymore since they are separated. The capillary time $t_{c}$ is the equivalent of the escape time in the necklace problem.

The blobs size distribution at $\mathbb{E}=1$ provides the drops size distribution $\mathbb{p}(d)$ at the end of the aggregation process, and is given by

$$
\begin{aligned}
\mathbb{P}(d) & =\mathbb{E}(d /\langle d\rangle) /\langle d\rangle \\
& =\mathbb{b}(d /\langle d\rangle) / B(1)
\end{aligned}
$$

identically to (9.10). The width of this distribution relative to its mean is given by $1 / \sqrt{\nu}$, a property of the Central Limit theorem (see also (9.45)). To conclude:

(i) Rough ligament: An initially corrugated ligament with $\gamma \gtrsim 1$ will give rise to the positively skewed distribution of sizes customary found in sprays described by (9.50) with a finite $\nu$, larger than unity. Given the link between the values of $\nu=1 /(\gamma-1)=\epsilon^{-2}$ and the geometry of the ligament made in Section 9.3, a corrugation amplitude is unlikely to be larger than half a ligament mean radius so that $\nu$ is typically equal to 4 in a very rough, but still cohesive ligament, and otherwise larger.

(ii) Smooth ligament: An initially uniform thread with $\gamma \rightarrow 1$ will give rise to a uniform collection of drops $\mathbb{E}(d /\langle d\rangle) \rightarrow \delta(d /\langle d\rangle-1)$, the Dirac Delta distribution being indeed the limit of a Gamma distribution with order $\nu \rightarrow \infty$. In that special limit, the number of drops $B(1)$ is identical to the initial number of blobs $B(0)$ covering the thread, since this number is left invariant through (9.40); in the language of Section 9.3, this results from the absence of a net longitudinal flow, and thus of any ligament coarsening in that case.

\subsubsection{Integer convolution and physical blobs 'à la Hauksbee'}

The fractional convolution dynamics in (9.40) has a clear meaning when $\gamma$ is interpreted as a ratio of lengthscales (9.45) which itself reflects a ratio of timescales (9.31). However, its meaning in terms of addition of blobs sizes is not immediately apparent, precisely because $\gamma$ is typically not an integer and that the relationship between partial convolution and the addition of random variables is not immediately apprehensible. In fact, it is, if one views the exchanges between the adjacent blobs defining the ligament cross-section thickness as mediated by smaller, physically identified blobs, somewhat 'à la Hauksbee' like in the drawing of in Fig. 2. In this vision, the ligament section is composed of $\nu$ parallel independent layers inside which the physical sub-blobs truly add their sizes at random during the coarsening process. Two adjacent sub-blobs sizes $d_{1}^{\prime}$ and $d_{2}^{\prime}$ in one of the $\nu$ layers add-up as

$$
d_{1}^{\prime}+d_{2}^{\prime}=d^{\prime}
$$

thus thickening the layer. The sub-blobs are independent so that $\left\langle d_{1} d_{2}\right\rangle=\left\langle d^{\prime}\right\rangle^{2}$. Obviously, $\langle d\rangle=\nu\left\langle d^{\prime}\right\rangle$, and since the layers are independent, the blob size distribution $\mathbb{b}(d, \mathbb{E})$ results from the sub-blobs size distribution $\mathbb{q}\left(d^{\prime}, \mathbb{E}\right)$ by a simple convolution process

$$
\mathbb{b}(d, \mathbb{E})=\mathbb{q}\left(d^{\prime}, \mathbb{t}\right)^{\otimes \nu}
$$

The analogue of the dynamics in $(9.40)$ for the Laplace transform of $\mathbb{q}\left(d^{\prime}, \mathbb{E}\right)$ now reads (Villermaux et al. 2004)

$$
\begin{aligned}
q(s, \mathbb{E}) & =b(s, \mathbb{E})^{\gamma-1} \\
\partial_{\mathbb{t}} q & =-(\gamma-1) B^{\gamma-1} q+\frac{\gamma-1}{3 \gamma-2} q^{2}
\end{aligned}
$$

which defines a complete, integer convolution equation of order 2 consistent with the complete sum in (9.54) leading, as is well known since Smoluchowski (1917), to a pure 
exponential distribution

$$
\mathbb{q}(d)=\frac{\nu}{\langle d\rangle} e^{-\nu \frac{d}{\langle d\rangle}}
$$

for the sub-blobs distribution $\mathbb{q}(d)$ and therefore, by $\nu=1 /(\gamma-1)$ convolutions, to the Gamma distribution in (9.50) for the ligaments blobs distribution.

The mean layers thickness $h_{0} / \nu=(\gamma-1) h_{0}$ is the mean free path of the internal motions across the ligament, those producing the random additions. This mean free path is necessarily smaller than $h_{0}$ itself as long as cohesion preserves the ligament integrity in the rearrangement period, thus suggesting the following bounds: $\gamma<2$, and $\nu>1$.

\subsection{Dissection of a jet}

The model we have described consists in visualizing a liquid ligament as a collection of interacting blobs, themselves made of sub-blobs aggregated at random. In this view, the number $\nu$ of virtual sub-blobs per ligament average radius is a function of the ligament roughness $\epsilon^{2} \sim \nu^{-1}$. Since, according to this vision, the whole is the sum of its parts, it is tempting to extract from a ligament a fraction of its volume and see how this dissected part has contributed to the construction of the whole.

Imagine a process by which smaller ligaments are stripped from a main ligament. After complete breakup, a fraction $\alpha$ of the drops will come from the stripped ligament, and the other fraction $1-\alpha$ from the main ligament. If the sub-ligament has been stripped at random from the main ligament core, the size distribution of its droplets is a Gamma distribution; let $\left\langle d^{\prime}\right\rangle$ be its average, $\nu$ its order and denote

$$
\Gamma(\nu, x=d /\langle d\rangle)=\frac{\nu^{\nu}}{\langle d\rangle \Gamma(\nu)} x^{\nu-1} e^{-\nu x},
$$

we thus have for the stripped ligament droplets size distribution

$$
\mathbb{q}\left(d^{\prime}\right)=\Gamma\left(\nu, d^{\prime} /\left\langle d^{\prime}\right\rangle\right)
$$

Then, the construction of Section 9.4.1 predicts, if the stripping operation has been made at random, that the size distribution of the main drops should be

$$
\begin{aligned}
\mathfrak{P}(d) & =\mathbb{q}\left(d^{\prime}\right)^{\otimes k} \quad \text { with } \quad k=\frac{\langle d\rangle}{\left\langle d^{\prime}\right\rangle} \\
\text { so that } \quad \mathbb{P}(d) & =\Gamma\left(k \nu, d /\left(k\left\langle d^{\prime}\right\rangle\right)\right)
\end{aligned}
$$

where $\langle d\rangle=k\left\langle d^{\prime}\right\rangle$ is the average drop size of the main drops from the original ligament. The stripped droplets distribution $\mathbb{q}\left(d^{\prime}\right)$ is the 'quantum' from which, by linear superposition, the distribution of the main ligament is produced. In other words, the stripped ligament is a specimen of those constitutive of the main ligament, across which they are randomly stacked. Similarly, although in a different context, the concentration distribution of random mixtures is the convolution of quanta, or 'solitary strips' formed by the stirring motions in the flow, which overlap at random by molecular diffusion in an additive fashion (Villermaux 2019).

The resulting overall drops size distribution $r(d)$ is thus the average of the two distributions $\mathbb{q}(d)$ and $\mathfrak{p}(d)$ weighted by the fraction $\alpha$

$$
\mathbb{r}(d)=\alpha \Gamma\left(\nu, d /\left\langle d^{\prime}\right\rangle\right)+(1-\alpha) \Gamma\left(k \nu, d /\left(k\left\langle d^{\prime}\right\rangle\right)\right)
$$

describing a bimodal distribution when $\left\langle d^{\prime}\right\rangle$ and $\langle d\rangle$ are sufficiently distant from each other, that is when $k$ is appreciably larger than unity. One distribution being generated by the other through the convolution operation in (9.61), it is shifted to larger sizes by a 

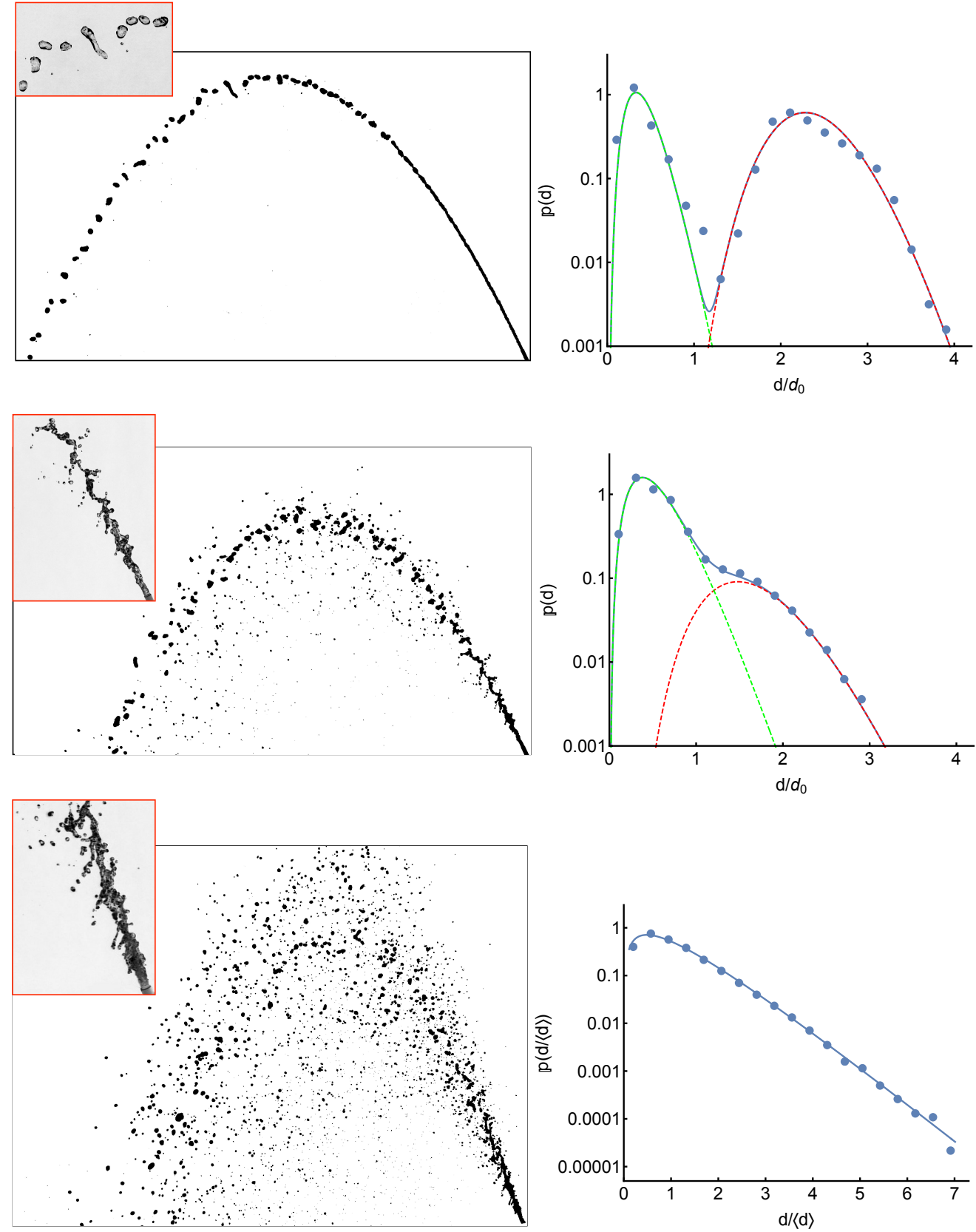

Figure 17. Fragmentation of a water jet (shot at an angle to the direction of gravity) as the level of turbulent fluctuations in the incoming stream is varied while the flowrate carried by the jet is kept constant. Top: A potential jet with very few internal turbulence fragmenting into the classical main drop/satellite drop size distribution. Curves are fit by (9.63). Middle: Stripping of transitional ligaments with $W e=\mathcal{O}(3-4)$ defined in (9.64) and fit by (9.63). Bottom: Fully stripped jet, all converted into a random set of sub-ligaments and fragments distribution given by $(9.82)$. 
factor $k$, and is also broader than the source distribution from which it originates. There is thus a rigid link between the two modes of the global distribution $r(d)$ : the orders of the stripped and of the main distributions are in the same proportion as their respective averages, namely $k$ as expressed by (9.61).

This dissection operation was realized experimentally making use of a turbulent jet. Indeed, if turbulent motions are superimposed on a mean jet flow, and if these are intense enough, they may overcome capillary cohesion to force the ejection of parts of the liquid from the jet core, a fact known for a long time (Schweitzer 1937; Wu \& Faeth 1995). The intensity of the (root mean squared) velocity fluctuation $u$ relative to the average jet velocity $U$ is measured by the opening angle $\beta$ of the fragmenting jet as $u=U \tan \beta$ when

$$
W e=\frac{\rho u^{2} d_{0}}{\sigma}>1
$$

with $d_{0}$ the jet diameter at the injector exit. When $W e<1$, the jet, although its surface is rough because of the liquid agitation, remains cohesive so that $\beta=0$ and no stripping occurs. For $W e \gtrsim 1$, ligaments are actually stripped from the main jet core, with a typical size, of order $\left\langle d^{\prime}\right\rangle \sim \sigma / \rho u^{2}$, which equilibrates cohesion and disgregation forces.

Observations of a moderately stripping jet with $W e=\mathcal{O}(3-4)$ indeed reveal bimodal overall drops size distributions $\mathbb{r}(d)$ well fitted by (9.63) with orders $\nu$ about 4 characteristic of strong corrugation, with an inter-modes convolution order $k \approx W e$, bridging the stripped ligaments size with the main droplets average size $\langle d\rangle$, itself of the order of the jet diameter $d_{0}$. Because the random aggregation scenario is constrained in distribution shape, Gamma orders and mean sizes ratios which should be all consistent with each other, a successful fit satisfying all the constrains constitutes a strong support to the theory.

For larger $W e$ (and larger jet Reynolds number), as the spectrum of the background turbulence in the jet broadens, the stripped ligaments are more broadly distributed in size, and possibly eject ligaments themselves so that the bimodality of the overall distribution disappears, leaving place instead to an essentially monotonically decreasing, exponential-like distribution. The distribution is now a compound, whose status we discuss in the next Section.

\subsection{Compound distributions}

Sprays are built from the breakup of ligaments, but all ligaments may not be identical in a given fragmentation operation. The poly-dispersity of the drops in a given spray may thus result both from the distribution of the drops coming from the breakup of a given ligament, and also from the distribution of the volumes carried in the population of the ligaments. A few particular cases exhibit a close-to-homogeneous population of ligaments so that the poly-dispersity of the drops sizes in the spray is all contained by that arising from the breakup of a single ligament (see e.g. Marmottant \& Villermaux (2004a); Bremond \& Villermaux (2005); Zhao et al. (2011); Vu \& Dumouchel (2018)). There is, however, no reason that this should be a general rule.

Let $\mathbb{q}\left(d / d_{l}\right)$ be the drop size distribution arising from the breakup of a ligament with size $d_{l}$ (this distribution belongs to the Gamma family with an order $\nu$ given the corrugation state of the ligament), and let $\mathbb{L}\left(d_{l} /\langle d\rangle\right)$ be the distribution of the ligaments diameters $d_{l}$ in a particular fragmentation protocol. Small ligaments will produce smaller droplets than larger ligaments. The overall distribution of sizes $d$ has thus two sources of variability, the one coming from the distribution of $d_{l}$, and the one coming from the distribution of $d$ for a given $d_{l}$. When these two factors are independent of each other, 
we expect the final droplets size distribution $p(d)$ to be given by a linear superposition of the two effects as

$$
\mathbb{p}(d)=\int \mathbb{q}\left(d / d_{l}\right) \mathbb{L}\left(d_{l} /\langle d\rangle\right) \mathrm{d} d_{l}
$$

making it clear that $\mathbb{p}$ is a mixture of both $\mathbb{q}$ and $\mathbb{L}$.

\subsubsection{Exactly computable ligament size distribution}

An important result was derived by Lhuissier \& Villermaux (2013) in the twodimensional version of the 'effervescent' atomization protocol (see Section 4.4). In this configuration, a liquid sheet is pierced at random locations in a nearly synchronous fashion. In this purely random, Poisson hole nucleation process, the sheet evolves, once the rims have all coalesced, towards a web of ligaments drawing exactly the edges of the Voronoï polygons from the nucleation sites. A series of geometrical constructions provides the distribution of distances $\lambda$ of the ligaments from the closest nucleation site and, more importantly regarding the present discussion, the distribution $\mathbb{L}\left(d_{l}\right)$ of ligaments diameters $d_{l}$, given by

$$
\begin{aligned}
& \mathbb{L}\left(x \equiv \frac{d_{l}}{\left\langle d_{l}\right\rangle}\right)=4 \Gamma\left(\frac{5}{4}\right)^{4} x^{3} e^{-\left[\Gamma\left(\frac{5}{4}\right) x\right]^{4}} \\
& \left\langle d_{l}\right\rangle=\frac{2 \sqrt{2}}{\pi^{3 / 4}} \Gamma\left(\frac{5}{4}\right) \sqrt{\langle\lambda\rangle h}
\end{aligned}
$$

where $\langle\lambda\rangle$ is given by the density of holes. This is a distribution highly peaked around its mean $\left\langle d_{l}\right\rangle$, the reasons for this being first the nonlinear transformation involving a square root in (9.67), and second that ligaments are built by an additive interaction of adjacent hole rims, thus averaging-out the disorder induced by the Poisson hole nucleation process. The consequences of this narrowness is that the poly-dispersity in the resulting spray is practically entirely due to the one coming from the ligament breakup per-se. Indeed, with nearly mono-sized ligaments

$$
\mathbb{L}\left(d_{l} /\langle d\rangle\right) \approx \delta\left(d_{l}-\left\langle d_{l}\right\rangle\right),
$$

the drop size distribution $\mathbb{p}$ is, as seen from (9.65), well approximated by a $\mathbb{q}$ itself, and is thus a Gamma distribution characteristic of ligament breakup (see (9.59))

$$
\mathbb{p}(d) \approx \Gamma\left(\nu, d /\left\langle d_{l}\right\rangle\right)
$$

of order $\nu$ found to be, for effervescent atomization, close to $3-4$. This holds as long as the collision of the rims forming the ligaments is not too violent. When this is no longer the case, breakup proceeds from a different scenario, examined in Section 10.3.

\subsubsection{Ligaments produced from corrugated ligaments: Bessel functions}

Often the ultimate ligaments, those forming the drops, are themselves stripped from other corrugated ligaments. This situation occurs when a source ligament is suddenly accelerated, or decelerated for instance, and therefore elongates into secondary ligaments from its initial protrusions. Relevant examples are found with drops impact (Villermaux \& Bossa 2011) where the fragments are ejected from the expanding rim of the flattened impacting drop, with rims collisions when the collision triggers the expulsion of ligaments transverse to the collision direction (Néel et al. 2020), a situation occurring generically in turbulent sheets randomly pierced by holes as in flat fan atomizers (Kooij et al. 2018).

In that case, the source ligament sizes $d_{l}$ are themselves distributed according to the distribution characteristic of corrugated ligaments, namely a Gamma distribution. Thus, 
with $\Gamma(\nu, x)$ given in $(9.59)$ and with

$$
\begin{aligned}
\mathbb{q}\left(d / d_{l}\right) & =\Gamma\left(\nu, d / d_{l}\right), \quad \text { for the elementary drop size distribution } \\
\mathbb{L}\left(d_{l} /\langle d\rangle\right) & =\Gamma\left(\mu, d_{l} /\langle d\rangle\right), \quad \text { for the ligament size distribution }
\end{aligned}
$$

we obtain from (9.65) a compound distribution of drops sizes with average $\langle d\rangle$ given by that of $\mathbb{L}\left(d_{l} /\langle d\rangle\right)$ as (Villermaux \& Bossa 2011)

$$
\mathbb{P}\left(\zeta=\frac{d}{\langle d\rangle}\right)=\frac{2(\mu \nu)^{\frac{\mu+\nu}{2}}}{\Gamma(\mu) \Gamma(\nu)} \zeta^{\frac{\mu+\nu}{2}-1} K_{\mu-\nu}(2 \sqrt{\mu \nu \zeta}),
$$

where $K_{\mu-\nu}$ is the modified Bessel function of order $\mu-\nu$, and where $\mu$ reflects the roughness of the distribution in ligament sizes $d_{l}$, while $\nu$ reflects that of the drops producing ligaments corrugations, in this construction. In the final expression (9.72), the roles of $\mu$ and $\nu$ are however interchangeable (the distribution is unchanged by the permutation $\mu \leftrightarrow \nu)$, both variabilities composing in an additive fashion, by principle: indeed, the square of the standard deviation $\left\langle\zeta^{2}\right\rangle /\langle\zeta\rangle^{2}-1$ of $p(\zeta)$ in $(9.72)$ is

$$
\frac{1+\mu+\nu}{\mu \nu}
$$

meaning, for instance, that the width of the final drop size distribution relative to its mean is given by that of the ultimate ligament breakup $(1 / \sqrt{\nu})$ when the ligaments are all alike $(\mu \rightarrow \infty)$, as in the effervescent atomization protocol (see Section 9.6.1 above, and Lhuissier \& Villermaux (2013)).

In the other limit where $\mu$ is moderate, the overall drop size distribution $p(d)$ is a compound of stable drops coming from the breakup of ligaments essentially exponentially distributed in size, a phenomenon encountered for instance with the 'soft' impact of drops bursting due to a relative motion in air (Villermaux \& Bossa (2009)). The large excursion tail of $p(d)$ is broader than an exponential, but narrower than a pure power law. Good fits using (9.72) were obtained by Néel et al. (2020) in strong rims collisions, and from well converged distributions in fragmenting turbulent sheets by Kooij et al. (2018, 2019).

\subsubsection{Random stripping}

Drops forming ligaments may be stripped from an agitated liquid bulk: hieratic motions pointing perpendicular to the liquid surface may, if they are strong enough to overcome capillary restoration at the interface, force the ejection of a ligament; we have given such an example in Section 9.5, where essentially only one class of ligaments is ejected from a weakly turbulent jet, the class for which the critical condition for ejection in (9.64) is met, corresponding to ligaments a couple of times smaller than the main jet from which they originate.

We envisage now the extreme limit where a broad spectrum of lengthscales is sufficiently excited in the liquid bulk to seed a broad spectrum of ejected ligaments sizes. This is not a sequential cascade of breakups scenario, but on the contrary a process of concomitant, parallel ejections, with different sizes. Let $\langle d\rangle$ be the mean drop size in the final spray. We know from Section 9.5 that a drop whose size $d$ is of the order of $\langle d\rangle$ comes from a ligament with diameter of the order of $\langle d\rangle$, and that this ligament is a random stack of $k$ quanta (see Section 9.5 for this terminology) of diameter $\langle d\rangle / k$. These ligaments may well be stripped from the liquid bulk on their own (provided, if they are driven by a velocity fluctuation $u_{k}$ such that $\langle d\rangle / k>\sigma / \rho u_{k}^{2}$ ), and breakup to contribute to the overall spray. 
We thus consider an a-priori very broad spectrum of ligaments sizes

$$
\langle d\rangle \frac{k}{\langle k\rangle}, \quad \text { for } \quad k=0,1,2 \ldots \infty
$$

with $\langle k\rangle$ some average number, larger than unity and assume that an elementary ligament drop size distribution $\mathbb{q}(d, k)$ is associated to each value of $k$, whose occurence in the collection of drops forming ligaments is given by a probability $p(k)$. Given the range we authorize for $k$, the original liquid volume is no more dissected as in Section 9.5, but pulverized into quanta which are left free to recombine according to a number frequency given by $p(k)$.

In order to reach sensible results easily, we further assume that the distribution $q(d, k)$ is very narrow (it is, strictly speaking, a Gamma distribution and we make its order $\nu \rightarrow \infty$, an assumption all the more justified that $\langle k\rangle$ is large)

$$
\mathbb{q}(d, k)=\delta\left(d-\langle d\rangle \frac{k}{\langle k\rangle}\right)
$$

and that the number of quanta $k$ is distributed at random in each ligament, meaning that $p(k)$ is a Poisson distribution

$$
p(k)=\frac{\langle k\rangle^{k} e^{-\langle k\rangle}}{k !}
$$

Obviously, the overall drop size distribution $p(d)$ writes, analogously to (9.63)

$$
\mathbb{P}(d)=\sum_{k=0}^{\infty} p(k) \mathbb{q}(d, k)
$$

and one checks that since $\sum k p(k)=\langle k\rangle$, we have $\int d \mathrm{p}(d) \mathrm{d} d=\langle d\rangle$. The Laplace transform of $\mathfrak{q}(d, k)$ in units of $d /\langle d\rangle$ reads

$$
q(s, k)=e^{-s \frac{k}{\langle k\rangle}}
$$

and the Laplace transform of $\mathrm{p}(d /\langle d\rangle)$ is thus

$$
\begin{aligned}
p(s) & =\sum_{k=0}^{\infty} p(k) q(s, k) \\
& =e^{-\langle k\rangle\left(1-e^{-s \frac{k}{\langle k\rangle}}\right)} \\
& \underset{\langle k \rightarrow 0}{\stackrel{s}{\langle}\rangle}\left(1+\frac{s}{\langle k\rangle}\right)^{-\langle k\rangle}
\end{aligned}
$$

which defines $\mathfrak{p}(d /\langle d\rangle)$ as a Gamma distribution of order $\langle k\rangle$

$$
\mathfrak{p}(x=d /\langle d\rangle)=\frac{\langle k\rangle\langle k\rangle}{\Gamma(\langle k\rangle)} x^{\langle k\rangle-1} e^{-\langle k\rangle x} .
$$

The accumulation of independent contributions from random ligaments produce Gamma distributions, through a construction mechanism similar to the one we have described to understand the emergence of Gamma distributions from the breakup of a single ligament in Section 9.4.1. The drop size distribution is universal in the $d /\langle d\rangle$ units, a fact recognized long ago; for instance, Simmons $(1977 a, b)$ notes that, for a large collection of industrial sprays, the distribution of sizes is, in shape, solely determined by its mean, and that its tail is well fitted by an exponential fall-off, as in (9.82). 
Such a scenario suits well to the fragments issuing from a turbulent jet at large Reynolds number $U d_{0} / \nu \sim 2 \times 10^{4}$ with initial large internal velocity fluctuations $u / U \approx 0.1$, and therefore with a Weber number defined in (9.64) appreciably larger than unity, of the order of $W e=13$. The corresponding range of excited scales, measured by the range of $k$ values is of course narrower than the infinite range in (9.74), but spans nevertheless a decade. These scales extend from $d_{0} / W e$ (the threshold for $W e>1$ ) defining $k_{\min }=$ $\left(d_{0} /\langle d\rangle\right)\langle k\rangle / W e$, to the limit of the capillary unstable range for a jet, namely $\pi d_{0}$, defining $k_{\max }=\left(d_{0} /\langle d\rangle\right)\langle k\rangle \pi$. By definition of the quantum, $k_{\min }=1$ so that $k_{\max }=W e$ and therefore

$$
\langle k\rangle=\frac{\langle d\rangle}{d_{0}} \frac{W e}{\pi}
$$

Since the measured mean drop size is $\langle d\rangle \approx 0.53 d_{0}$, the expected value of the mean number of quanta per ligament is $\langle k\rangle=2.1$, indeed slightly larger than unity. The observed overall drop size distribution $\mathrm{p}(d /\langle d\rangle)$ is very well represented by the Gamma distribution in (9.82), of best fitted order $\langle k\rangle \approx 1.9$.

\subsubsection{A note concerning the mean}

The aggregation theory and its variants we have developed in this chapter predict the shape of the drops size distribution in units of their mean (i.e. $d /\langle d\rangle$ ). The only thing this theory does not predict from its intrinsic ingredients is the mean diameter $\langle d\rangle$. This may look odd since in fragmentation studies, one is used to see first a discussion about the mean size, then some considerations on the fluctuations about the mean, and possibly general statements on the shape the fragments distribution should have. We have in fact taken the problem in reverse, in addressing the microscopic process at play for building the fragments distribution in the first place. We have discovered that it is an aggregation process from an elementary quantum, to macroscopic fragments; from that observation, we have inferred what the distribution shape should be. It is therefore clear why the average fragment size cannot be predicted from the only ingredient that fragments are constructed by an inverse cascade of aggregation; for this, one has to decide when the inverse cascade stops, and this decision requires an additional physical ingredient.

Understanding the distribution shape and the mean size are, in fact, two separate discussions: Think, for instance, of the velocity distribution in a gas: the Maxwell shape relies on general arguments of symmetry and homogeneity of space (Maxwell 1867) while the mean speed is independently fixed by temperature; Similarly, the shape of Planck's spectral radiance of the black body (Planck 1901) solely relies on the fact that light emission is quantized, while the wavelength of maximal emission (Wien's displacement law $\left.\lambda_{\mathrm{m}} T \sim \hbar c / k_{\mathrm{B}}\right)$ expresses thermal equilibrium. The magnets necklace fragmentation problem in Section 9.2 is also exemplary in this respect; there, the cascade interrupts when the aggregation time of the mean fragment becomes infinite, thus setting its value. This divergence reflects the radial expansion geometry particular to the necklace problem. Different expansion rates lead to different mean fragments sizes, but the distribution shape is universal when fragments sizes are measured in units of their mean.

Similarly, for the fragmenting turbulent jet whose distribution has been computed in (9.82), the new ingredient one has to put in to determine $\langle d\rangle$ is, as in Eqs. (9.19) and (9.31), a ratio of timescales indicating the end of the cascade. Above the stripping transition $(W e \gtrsim 1)$, blobs recombine on a capillary timescale as they separate in the diverging base flow. A mean size is attained when the recombination time $\sqrt{\rho\langle d\rangle^{3} / \sigma}$ 
balances the substrate divergence time $d_{0} / u$, so that we expect

$$
\frac{\langle d\rangle}{d_{0}} \sim W e^{-1 / 3}
$$

where the Weber number is the one from (9.64) constructed on the divergence velocity $u$. The structure of (9.84) is, not surprisingly, identical to the one in (2.13).

Recombination equilibrates substrate divergence for a jet expanding freely but, inline with the discussion we made about the viscous dissipation scale in the Taylor-Culick rim recession problem in Section 3.2.1, the above reasoning leading to (9.84) can be adapted to a confined, turbulent flow where now the reference time is the divergence time $\langle d\rangle / u(\langle d\rangle)$ at the scale $\langle d\rangle$ itself when the divergence velocity is scale-dependent, like within the inertial range of scales at large Reynolds number where $u(d) \sim u\left(d / d_{0}\right)^{1 / 3}$. In that case one anticipates that (Kolmogorov 1949)

$$
\frac{\langle d\rangle}{d_{0}} \sim W e^{-3 / 5},
$$

a relationship which was actually found by Hinze (1955) to fit well the maximal droplets sizes in emulsions recorded originally by Clay (1940) using various apparatus, including a turbulent pipe, and a Taylor-Couette flow (see Lemenand et al. (2003) for a reedition of this type of experiments).

\section{Formation of 'fines', direct cascades}

Conventionally viewed as a process where the arrow of time points towards ever smaller sizes by the repeated action of various stresses, the fragmentation of a cohesive material results, on the contrary, from an inverse cascade of aggregations at the microscopic level, starting with the smaller atoms, up to stable bigger fragments (Section 9, in particular). The cascade is interrupted when separation forces overcome cohesive forces, leaving the broken material in a dispersed state whose statistics is interpretable from first principles in simple situations like for the magnets necklace in Section 2.3.

The reason for this regrettable confusion is to a large part attribuable to an authoritative argument personified, without his knowledge, by A. N. Kolmogorov. He produced in 1941 a short note (not to be confused with the three celebrated notes on turbulence that same year), written in german, in the USSR academy of sciences Doklady motivated by discussions he had had with a colleague (N. K. Razumovsky) about the size statistics of fragments during ore processing (Kolmogorov 1941b). This colleague had found that the logarithm of the particles sizes during crushing and grinding was well represented by a Gaussian. In these kinds of processes, a cohesive material breaks sequentially because it is exposed to a successive series of solicitations or stresses involving repeated impacts with an object (using jaw crushers, hammer mills, ball mills etc... see Coulson et al. (2002)), or between the fragments themselves; the size distribution builds-up step by step as the number of uncorrelated, repeated solicitations increases. Kolmogorov came up with an elegant and simple idea where the size of any daughter particle derives from that of the mother particle through a product series of random multipliers (smaller than unity), yielding the celebrated 'lognormal distribution' along with the mental image of the 'direct cascade towards smaller scales' (fortunately not accompanied, unlike for turbulence, by a poem paraphrasing Swift...). This argument, probably because it is clever and straightforward, partly also because it emanated from Kolmogorov, has been since then blindly transported by many (in solid fragmentation, and liquid atomization, see the critical review in Villermaux (2007)) to situations where the lognormal distribution 
(i)

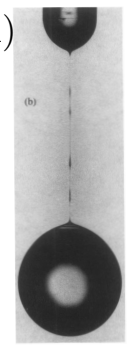

(iv)

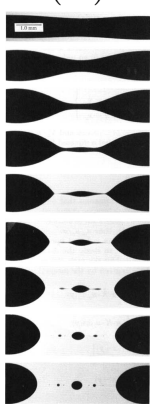

(ii)

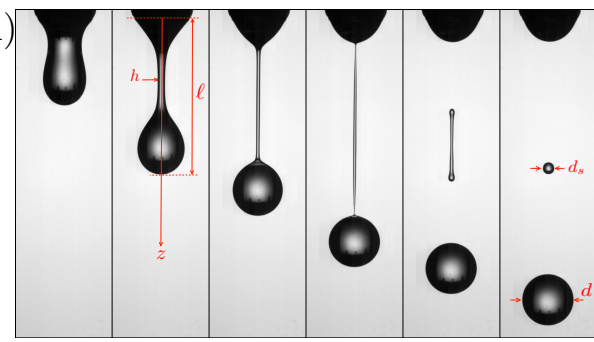

(

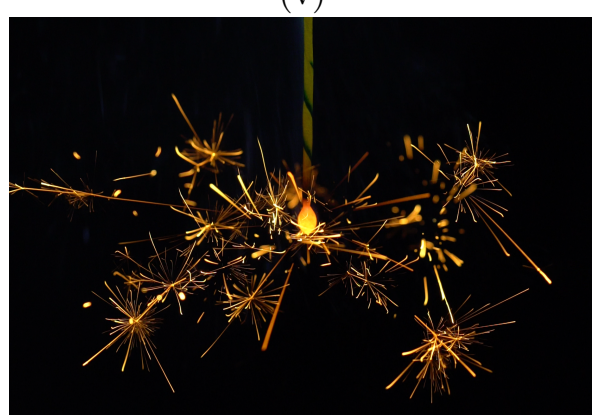

(iii)
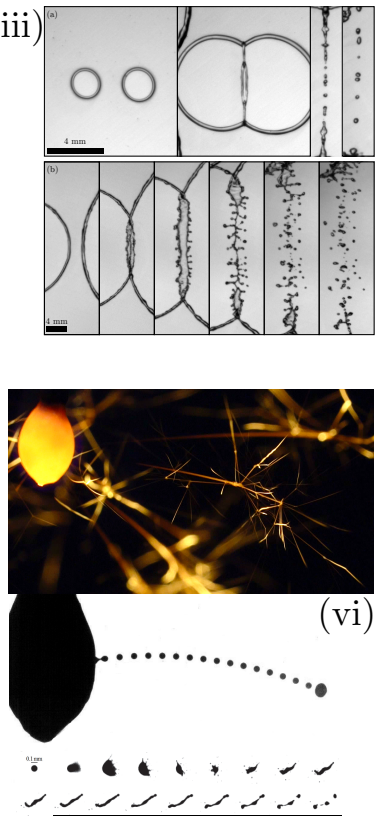

Figure 18. Examples of direct cascades and production of 'fines'. (i) Iterated instabilities on a viscous thread (Brenner et al. 1994). (ii) Formation of a satellite drop on a viscous stretching ligament (Villermaux et al. 2013). (iii) Production of 'fines' by the impact of two liquid rims opening on a liquid sheet (Néel et al. 2020). (iv) Breakup of a viscous liquid bridge featuring three steps of consecutive capillary instabilities (Tjahjadi et al. 1992). (v) Cascade of incandescent droplets in fireworks where a daughter drops is the mother drop of the next step and, (vi) detail of the trace of the successive droplets and of their ligament-mediated fragmentation (Inoue et al. 2017).

has no reason to be, simply because there is no repeated sequence of mechanical action at all, but instead a single action; and in that case there is indeed a cascade, but it is in reverse as explained in Section 9.

With this caution in mind, we give below a few examples of direct cascades in nature where there is at least one step of size reduction from a big object, to a smaller one. When the size reduction process persists over multiple steps, very small particles, sometimes called 'fines', are produced.

\subsection{Iterated thread fission, coalescence cascade}

Capillary instabilities responsible for the breakup of an initially smooth ligament may follow each other sequentially: the thin neck linking two neighbor bulges in the process of separating is locally a liquid thread as well, which may also suffer a capillary instability, itself producing bulges linked by a thinner ligament etc... The process was already visible in Plateau's experiments with olive oil (Plateau 1849) and it has been since then identified in related contexts involving viscous fluids (Brenner et al. 1994; Wong et al. 2004; Villermaux et al. 2013), not to mention viscoelastic fluids where the phenomenon is the rule (Oliveira \& McKinley 2005; Keshavarz et al. 2020). The consequence of this scenario is the typically bimodal character of the drop size distribution (the small drops are called 'satellites'), which present two broad but well separated peaks (see e.g. Basaran et al. (2013) in the context of inkjet printing), and even a fractal sequence of iterated peaks when the cascade has the chance to persist over multiple steps (Tjahjadi et al. 
1992), which is rare. With water however, the capillary breakup is so fast that the phenomenon is virtually absent, unless altered by ad-hoc perturbations (Lafrance \& Ritter 1977). Surfactants on the other hand foster a counter Marangoni stress in the pinch-off region which delays breakup, and favors a multiple step cascade (Kamat et al. 2018). Rough ligaments with random pre-existing corrugations follow a different, opposite route (Section 9).

Similar capillarity driven cascades are found in the hesitant coalescence of a drop on a liquid pool (Thoroddsen \& Takehara 2000) presenting remarkably up to six steps, the bursting of viscous bubbles (Herman \& Mesler 1987; Bird et al. 2010), or the gravitational settling of heavy drops in a lighter liquid (Thomson \& Newall 1885; Arecchi et al. 1996).

\subsection{Imploding cavities}

We have explained in Section 8.1.2 how the collapse of a cavity at the surface of a liquid, concentrating the fluid impulse into a narrow region of space at the base of the cavity produces a fast jet, whose breakup forms droplets which may be very fast, and tiny. This is an only one-step cascade, but it is emblematic of these configurations bridging the large (the cavity size) with the small (the final droplets).

There was a rule of thumb dating back to Blanchard (1963) saying that, for a cavity made from a bubble of size $R$ at the surface of water, the jet speed at bubble burst is roughly $R / t_{c}(R) \sim \sqrt{\sigma / \rho R}$ and the droplets size is about $0.1 R$. Recent research on the topic revived by Ghabache et al. (2014a) has shown that things are much more complicated, and that a crucial partner in the process is the capillary waves train emitted at bubble collapse, which travels along the cavity surface and which is the main focussing mechanism triggering jet emission. Surprisingly, liquid viscosity fosters even faster, and thinner jets (Ghabache \& Séon 2016); ejection velocities can be twenty times as large as the capillary velocity. An intense debate has followed (Gordillo \& Rodríguez-Rodríguez 2019) to describe the appropriate scaling laws.

\subsection{Collision of rims}

The fines are these smaller droplets that are produced from an auxiliary mechanism besides the formation of the standard drops in a fragmentation process. Néel et al. (2020) report their formation in a controlled experiment which isolates an individual fragmentation protocol: the collision of two rims bordering growing adjacent holes on a liquid sheet. The standard drops come from the capillary breakup of the fused rims. Occasionally, the rims collision is strong enough to trigger a new, splash-like mechanism, producing an expanding lamellae perpendicular to the main sheet, which destabilizes into finer drops, first discovered by Lhuissier \& Villermaux (2013) in the context of sheet breakup, more precisely for sheets which nucleate multiple holes (Section 4.4). Because surface tension forces are not balanced at their rim, holes grow in diameter and eventually merge with neighboring growing holes in the sheet plane. The merging event may simply consist in an inelastic coalescence of the rims or, if the collisional rims are sufficiently fast and thick, may trigger a splash. This is the cylindrical version of the binary impact of spherical drops problem (Bradley \& Stow 1978; Ashgriz \& Poo 1990; Roisman 2004). The phenomenon is very similar to the one known for drops impacting a solid (see Worthington (1876); Riboux \& Gordillo (2015) and the review in Josserand \& Thoroddsen (2016)), or a layer of the same liquid (Thoroddsen 2002; Agbaglah \& Deegan 2014), as well as for the entry of a solid in a water pool (Worthington 1908; Wagner 1932). It produces, right upon impact, a thin fast lamella ejected from the impact point in the direction perpendicular to the collision plane, at the edge of which small (compared to 
the impacting rims diameter) droplets are formed; this is the way fines are produced by this one-step cascade process (see Section 7.1.1).

Note that the adjectives 'fine' as opposed to 'coarse', or 'small' versus 'big' have no concrete reality in the absence of an absolute reference scale. Their use reflects more a personal appreciation. For instance, the drops emitted from the jet sparking at the collapse of a cavity (the so-called Worthington jet, that he himself found 'exquisite' and 'graceful', see Worthington (1908), p. 78 and which is usually qualified as 'fast' (Gordillo \& Rodríguez-Rodríguez 2019)) are sometimes considered as 'tiny' (Ghabache \& Séon 2016) while Woodcock et al. (1953) considered them as 'giant' nuclei. It is true that when the cavity is the one of a bubble at the surface of a liquid pool, the jet drops are larger than those formed from the disruption of the bubble cap (Spiel 1994). Similarly, the threshold size of $100 \mu \mathrm{m}$ defining fine droplets for spray drift in agriculture relates to normal wind, humidity, temperature conditions and typical fields dimensions. These may vary, and hence will the threshold. Interestingly, a similar $100 \mu \mathrm{m}$ threshold has been identified in human exhalations like sneezing (Wells 1955), a fact which clearly underlines its anthropomorphic nature.

\subsection{Reactive droplets, fireworks}

Known since the Edo period (1603-1868) in Japan, one of the most popular handheld fireworks has been the Senkou-Hanabi. This traditional firework generates light streaks similar to branched pine needles, with ever smaller ramifications. These streaks are the trajectories of incandescent reactive liquid droplets bursting from a melted powder (so-called 'black powder', a mixture of carbon C, sulfur S, and potassium nitrate $\mathrm{KNO}_{3}$ ). Inoue et al. (2017) have uncovered the detailed sequence of events, which involve a chemical reaction with the oxygen of air, thermal decomposition of metastable compounds in the melt, gas bubble nucleation and bursting, liquid ligaments and droplets formation, all this occurring in a sequential fashion proceeding over up to eight generations.

In contrast to situations where energy for breakup is injected in the system from the start, and decays as it produces an inverse cascade of coalescences between fragments (Section 9) like, for instance, the elements of the Universe synthesized in stars do (Alpher et al. 1948; Burbidge et al. 1957), this direct cascade is self-sustained by a constant injection of heat from the reaction of carbon with the oxygen of air. It is this exothermic reaction which, at every step of the cascade, initiates the nucleation of gas cavities in the metastable liquid droplets. Like in atom fission (Born 1937; Bohr 1939), droplets divide sequentially and self-similarly, down to an elementary brick where heat release and losses equilibrate, marking the end of the cascade.

A similar phenomenology is encountered in the combustion of multi-component fuel droplets with high volatility differential (Avedisian \& Andres 1978; Rao et al. 2017), or with emulsions (Rao \& Basu 2020). In both cases, the volatile phase nucleates a vapor bubble within the continuous liquid phase of the droplet, a bubble which burst and ejects a ligament when reaching the droplet surface.

\subsection{Minimal fragment size}

A direct cascade is always ultimately interrupted by a dissipative physical ingredient (viscosity, heat conduction etc...) whose relative importance grows as sizes decay, and which finally becomes dominant. In that case, the corresponding limit fragment size pertains to the range of the continuum scales (see e.g. Kendall (1978)). However, if the cohesive material is initially dismantled into its elementary atoms (this condition 
requires that the microscopic particle Weber number in (2.12) is larger than unity), as in the magnets necklace problem, then fragments as small as the elementary brick can be formed (provided they escape, by chance, the aggregation dynamics forming the macroscopic final fragments), precisely because they are formed from the start, and not at the end of an interrupted direct cascade. Such a scenario is besides compatible with the experiment of Kroto et al. (1985) who synthesized a complex molecular architecture (Backminsterfullrene) from elementary bricks laser-ablated from a piece of graphite (see also Section 7.3).

The heuristically exciting question of the minimal fragment size in comminution, the science of size reduction by crushing and grinding, certainly requires more attention.

\section{Energetics of fragmentation}

It is tempting, in front of a complicated problem where many intermingled effects are at play, to resort to general principles, an attitude sometimes adopted in despair in fragmentation mechanics. Because one must be extremely cautious in retaining the relevant ingredients when working these principles out, their use is most of the time hazardous. This is particularly the case with energy whose interest in science -because it is globally conserved- was first advocated by von Helmholtz (1847) in a celebrated pamphlet.

\subsection{Misconceptions}

We have already seen in Section 3.2.1, about the Taylor-Culick recession speed of a liquid film edge, how a naive because incomplete application of a principle of conservation between kinetic and surface energy, disregarding dissipation (which represents exactly half the total surface energy swept by the film edge) leads to a flawed prediction (in absolute value, not in scaling dependencies). The error is even worse if one considers the recession of an end-bulge attached to a cylindrical liquid ligament: the surface tension force not only feeds kinetic energy and dissipation, but also needs to work against the Laplace pressure $\sigma / h$ inside the ligament (with radius $h$ ). The equivalent of (3.13) now writes $\dot{P}=2 \pi h \sigma-\pi h^{2}(\sigma / h)$ and $\dot{m}=\rho \pi h^{2} v$, leading to $v=\sqrt{\sigma / \rho h}$ while a balance surface/kinetic energy would give $2 \sqrt{\sigma / \rho h}$, involving an error by a factor 2 due to the neglect of both dissipation and internal pressure work.

Fluid particles arrested at a rim dissipate their kinetic energy. The energy dissipated by a mass $m$ experiencing a velocity drop $\Delta u$ is equal to the kinetic energy of the velocity difference $\frac{1}{2} m(\Delta u)^{2}$, a result know since Borda (1763) (see also Appendix B in Villermaux \& Bossa (2011)). Consider for instance the dynamics of the expandingretracting sheet formed by the impact of a liquid drop with kinetic energy $E=\frac{1}{2} m u_{0}^{2}$ such that $\tau=\sqrt{m / \sigma} \gg r_{0} / u_{0}$, that is $W e \gg 1$ (Section 7.1). The sheet radial velocity is $u(r, t)=r / t$ and the velocity decrement at the rim in $r=R(t)$ is $\Delta u(t)=u(R, t)-\dot{R}$ where $R(t)$ is given by (7.9). Since the fate of every mass particle in the drop is to pass through the rim, estimating the dissipated energy $\Delta E$ at the rim amounts to estimate the value of $\overline{(\Delta u)^{2}}$ over the sheet oscillation cycle (lasting for about $\tau$ ) to form $\Delta E=$ $\frac{1}{2} m \overline{(\Delta u)^{2}}$. Since $\tau$ is independent of $W e$, so is $\Delta E / E$. The maximal velocity difference is $\Delta u(t / \tau=1 / 2)=u_{0} / 2$ (ocuring between the sheet maximal extension in $t / \tau=1 / 3$ and the cancellation of the rim deceleration in $t / \tau=2 / 3)$ and we have

$$
\frac{\Delta E}{E} \approx \frac{1}{4}
$$

The net amount of kinetic energy dissipated in the rim represents a fixed fraction of the 
initial kinetic energy of the drop of the order of $25 \%$, independent of $W e$. The rest splits into the kinetic energy of the fragments, and their surface energy.

In fact, the difficulty with this concept is that we know that energy is globally conserved among its various forms (heat, kinetic, radiations, surface, elastic...), but we have no principle to decide how the system will favor each in a particular situation. Sometimes, by luck, the problem can be completely solved from the principles of conservation of mass and momentum alone, like for the Taylor-Culick problem, and the energy budget is then computed a-posteriori. An a-priori energy approach is useful when only two kinds of energies are essentially at play, in quasi-steady situations like for the viscous version of the Taylor-Culick problem (which involves a tradeoff between viscous dissipation and surface destruction, see Section 3.2.2), or to estimate the limit stress for crack opening in a brittle elastic material (a tradeoff between stored elastic energy and surface creation, the reality of a surface energy being, in that context, questionable (Griffith 1921; Bourdin et al. 2008)), but when inertia is an ingredient of the problem, things are less straightforward. The opening of cracks is accompanied by branchings, acoustic, light emissions etc... which are all sinks of energy (Lawn 1993; Fineberg \& Marder 1999), yet unpredictable for the time being. It is however well known that in typical solid size reduction processes, only $10 \%$ of the input power is usefully employed at creating new interface area, the rest being dissipated as heat (see Ch. 2 in Coulson et al. (2002)).

This proportion is even larger than the one measured in the fragmentation of a stationary Savart sheet into droplets (Section 4.1). From the measured speed of the rim ejecta, Taylor $(1959 c)$ estimated that $98 \%$ of the incident kinetic energy 'disappeared in the region where the drops were formed' while Clanet \& Villermaux (2002) find that only $10 \%$ of the initial energy remains in the fragments, most of it in a kinetic form. The dissipated energy is very sensibly related to the fragments velocity decrement $\Delta u$ from the incident velocity $u_{0}$ since

$$
\frac{\Delta E}{E}=\left(\frac{\Delta u}{u_{0}}\right)^{2}
$$

giving, according to the ejection velocity at the cusps tips bordering the indented sheet found in (4.7), $\Delta E / E=(4 / 5)^{2}=64 \%$.

If from these examples, one sees that there is not a sharp consensus on the exact figure representing the amount of energy actually employed at creating new surface area, a certitude is that it systematically represents a small fraction of the available mechanical energy. It is also useful to note that its relative amount, at least its order of magnitude, is close to the ratio of the separation energy of the molecules, to the latent heat in the evaporation of water, which is of the order of 20\% (see Agrawal \& Menon (1992); the comparison of these two forms of energy has been historically made to infer the size of molecules, as recalled in Rowlinson (2002)).

The 'sentimental' vision which consists in attributing arbitrarily the entire available input energy to the surface energy of the desired products (the fragments), first formalized by von Rittinger (1867) usually fails dramatically not only in order of magnitude, but also in law. We have seen an example above concerning the impact of a drop and inherent dissipation by rims, another is provided by the liquid shell explosion studied in Section 5.1.1: the total available energy is the one released by the chemical reaction. For a given number of moles $m$ of a (stoichiometric, say) mixture enclosed in the bubble with radius $R_{0}$, one knows that the energy released will be $E=-\Delta H \times \frac{2}{3} m$ (the formation enthalpy of water vapor by $\mathrm{H}_{2}+\frac{1}{2} \mathrm{O}_{2} \longrightarrow \mathrm{H}_{2} \mathrm{O}$ is $\Delta H=-241 \mathrm{~kJ} / \mathrm{mol}$ ). An 'à la von Rittinger' temptation is to equate this energy with the one necessary to breakup the liquid shell, and further assume that this fracture energy is itself reflected by the final surface energy 
of the fragments. If $\Omega=4 \pi R_{0}^{2} h$ is the volume of the liquid shell, $N$ the number of fragments and $\langle d\rangle$ their average size, we thus have $\Omega \sim N\langle d\rangle^{3}$, and from the application of the (flawed) conservation principle stated above, $E \sim N\langle d\rangle^{2} \sigma$, from which one would predict

$$
\langle d\rangle \neq \frac{\Omega \sigma}{E}, \quad N \neq \frac{(E / \sigma)^{3}}{\Omega^{2}}
$$

The symbol $\neq$ signifies clearly the absurdity of this prediction since, with $R_{0}=2 \mathrm{~cm}$, one has $\frac{2}{3} m \simeq 10^{-3}$ moles at atmospheric pressure, and thus $E \simeq 221$ Joules giving according to $(11.3)$ a fragment size $\langle d\rangle$ of the order of $10^{-12} \mathrm{~m}$, smaller than the interatomic distance...! The reason for this major failure is that this caricature, which is even wrong in scaling dependencies, disregards several other sinks of energy, like the work done by the burnt gases to push the unburnt, the shell, and the outside air, thus communicating to them a substantial kinetic energy, but also disregards the highly dissipative dynamics of the rims and ligaments breakup on the liquid shell, in particular.

\subsection{Efficiency}

The inaugural example of the expanded necklace of magnets (Section 2.3) provides again a way to address the question of the partition of energy in its various forms in an unambiguous way. The input energy $\frac{1}{2} N m V^{2}$, entirely in a kinetic form, is the kinetic energy of each bead, all expanding at velocity $V$ times the number of beads $N$. The surface energy is the energy spent at opening a link, of order $a \phi(a)$, times the number of opened links $N /\langle n\rangle$, itself given by the final mean number of beads per fragment $\langle n\rangle$ in (2.13). We thus define, as is customary in the context of comminution, an efficiency $\mathcal{E}$ as the ratio of the surface energy to the input kinetic energy (see Vledouts et al. (2015) for references and a slightly different description)

$$
\mathcal{E}=\frac{\frac{N}{\langle n\rangle} a \phi(a)}{\frac{1}{2} N m V^{2}},
$$

this expression holding when at least one bond has opened, otherwise $\mathcal{E}=0$ since no surface creation has occurred. This is what happens when the impact velocity is smaller than the critical velocity $V_{c}$ in $(2.5)$ below which the necklace simply coalesces back to its initial state, remaining un-fragmented.

Above that critical impact velocity, the fragments velocity is given by $\sqrt{V^{2}-V_{c}^{2}}$ in (2.6). It is smaller than $V$, but non-zero. We thus have here a fragmentation process where the initial energy splits into surface energy, and kinetic energy; moreover, the ratio of these two forms of energy depends on the strength of the initial expansion. In terms of the particle Weber number $W e$ in (2.12) based on the divergence velocity at the scale of the elementary beads $u_{1,1}=2 \pi V / N$, one sees, recalling that $\rho \sim \mathrm{m} / \mathrm{a}^{3}$ and $\sigma \sim \phi(a) / a$, that the efficiency

$$
\mathcal{E} \sim\left(N^{3} W e\right)^{-2 / 3}
$$

is a decaying function of $W e$. For strong impacts when $W e$ approaches 1 , the efficiency $\mathcal{E}$ which scales as $1 / N^{2}$ goes to zero as more isolated beads expand (for larger $N$ ), essentially keeping their initial kinetic energy, the energy cost for opening the bonds linking them being in comparison negligibly small.

Starting from $\mathcal{E}=0$ when $V=V_{c}$ (that is $W e \sim 1 / N$ ), the efficiency thus first increases as $W e$ increases, and then decreases according to (11.5). Vledouts et al. (2015) give the complete dependence of $\mathcal{E}$ on $W e$ and $N$, showing that it is

(i) non-monotonic, 
(ii) always way below unity in absolute value,

exhibiting a maximum (about $\mathcal{E} \approx 0.3$ ), thus contradicting von Rittinger's sentimental principle according to which it should be constant, and of order unity. The energy budget computed a-posteriori in this particular case demonstrates the vacuity of 'energy based approaches' in fragmentation in general.

Other lumped descriptions (Grady 1982, 2006; Sultanov \& Yarin 1990; Bazant \& Caner 2013) have heuristically invoked the expansion rate of a breaking material to put it in relation with some measure of its cohesion in order to derive fragments sizes, and others have noticed that there are distinct regimes of fragmentation of a stressed material where the ejection velocity of the fragments depends on the initial conditions (Wildeman et al. 2017). The academic exercise of the expanded necklace of magnets offers a precise picture for which error does not escape from proof.

\section{Appendix A. Catalogue of relevant instabilities}

A list of the instabilities relevant to the phenomena discussed in this review is provided below in a digest form, including a presentation of the physical mechanism at play, the scalings for the length and timescales involved, and the full dispersion equation whose derivation can be found in the appropriate references mentioned in each case.

In most situations discussed below, instability occurs between two phases separated by an interface spreading along the $x$ axis with position in the $y$ direction $\xi(x, t)$ made of a superposition of Fourier modes

$$
\xi(x, t) \sim \sum_{k} \xi_{k} e^{i k x-i \omega t}
$$

whose dynamics is studied mode by mode (in short, $\xi(x, t) \sim \xi e^{i k x-i \omega t}$ ) through the dispersion relation $\omega(k)$. The range of unstable $k$-modes is the one for which $\operatorname{Re}[-i \omega(k)]>0$, defining a growth rate, whose square is given by

$$
\frac{\ddot{\xi}}{\xi}=-\omega^{2}
$$

Reasonings in the physical space involve the wavelength $\lambda=2 \pi / k$. In regions where the fluids are initially at rest, or move at a uniform translation velocity $u_{0}$ along the interface, the perturbed displacement field $\mathbf{u}$ obeys at lowest order $\partial_{t} \mathbf{u}+u_{0} \partial_{x} \mathbf{u}=-\nabla p / \rho$ and since for incompressible fluids $\nabla \cdot \mathbf{u}=0$, the pressure is harmonic $\nabla^{2} p=0$. This property sets the spatial structure of the perturbations

$$
\mathbf{u}, p \sim e^{ \pm k y} e^{i k x-i \omega t}
$$

When the interface is distorted over $\lambda$ in an unbounded domain, the fluids move over $\lambda$ away from it so that the mass in motion in the plane is proportional to $\rho \lambda^{2}$.

\section{A.1. Rayleigh Taylor}

A layer of heavy fluid (density $\rho_{1}$ ) is superimposed on a layer on lighter fluid (density $\rho_{2}$ ) perpendicular to the direction of a body force $g$ (the acceleration of a non-galilean frame, or gravity), pointing downwards. We denote $\Delta \rho=\rho_{1}-\rho_{2}>0$. The instability mechanism is that of free fall. The Archimedes force $\Delta \rho g \xi \lambda$ feeds the fluids inertia $\left(\rho_{1}+\right.$ $\left.\rho_{2}\right) \lambda^{2} \ddot{\xi}$ so that, in the absence of restoring forces at the interface, the square of the growth rate growth rate is

$$
\frac{\ddot{\xi}}{\xi} \sim \frac{\Delta \rho}{\rho_{2}+\rho_{1}} \frac{g}{\lambda}
$$


When the heavy fluid is confined in a layer of thickness $h$ suspended on a horizontal rigid wall, mass conservation $u h \sim \lambda \dot{\xi}$ in the heavy layer with horizontal velocity $\left(\rho_{1}+\rho_{2}\right) \partial_{t} u \sim$ $-\partial_{x} p$ leads, with $\partial_{x} p \sim-\Delta \rho g \xi / \lambda$, to

$$
\frac{\ddot{\xi}}{\xi} \sim \frac{\Delta \rho}{\rho_{2}+\rho_{1}} \frac{g h}{\lambda^{2}}
$$

If the fluids are very viscous, the Archimedes force now equilibrates viscous stresses, of order $\eta \dot{\xi} / \lambda$ so that we have now a first order relaxation dynamics

$$
\frac{\dot{\xi}}{\xi} \sim \frac{\Delta \rho g}{\eta} \lambda
$$

There is, in the cases envisaged above, no mode selection. In particular, small length scales grow infinitely fast in the inertial limit of (A 4). Since these induce large interface curvature $\xi / \lambda^{2}$, we expect that the corresponding Laplace force $\sigma\left(\xi / \lambda^{2}\right) \lambda$ subtracted to the Archimedes force will limit their growth. The force balance then leads to

$$
\left(\rho_{1}+\rho_{2}\right) \frac{\ddot{\xi}}{\xi} \sim \frac{\Delta \rho g}{\lambda}-\frac{\sigma}{\lambda^{3}}
$$

The range of unstable length scales is now limited by the capillary lengthscale $1 / a^{2}=$ $\Delta \rho g / \sigma \equiv k_{c}^{2}$, defining the capillary wavenumber $k_{c}$. The complete dispersion equation of this instability (between infinite, inviscid phases) is

$$
\left(\rho_{2}+\rho_{1}\right) \omega^{2}=-\Delta \rho g k+\sigma k^{3}
$$

The most amplified wavenumber is $k_{\mathrm{m}}=k_{c} / \sqrt{3}$, with growth rate $\sim \Delta \rho /\left(\rho_{2}+\rho_{1}\right) \times \sqrt{g k_{\mathrm{m}}}$.

In the opposite stable configuration $\left(\rho_{1}=0, \rho_{2}=\rho\right.$ for example), we recover the dynamics of dispersive gravity waves $\omega \sim \sqrt{g k}$ for $k \ll k_{c}$, and the one of capillary waves $\omega=\sqrt{\sigma k^{3} / \rho}$ for $k \gg k_{c}$ whose group velocity $\partial_{k} \omega$ is decreasing as $\sqrt{g / k}$, or increasing as $\sqrt{\sigma k / \rho}$ as a function of $k$, respectively.

This instability was first described in the form of (A 8) by Lord Rayleigh (1883), who also envisaged the case where the density jump between the phases is smooth and occurs over a non-zero thickness layer. It was first investigated experimentally by Lewis (1950). It is described in Lamb (1932) and Landau \& Lifshitz (1987) including the viscous corrections, in Limat (1993) for a finite heavy layer thickness suspended by a wall, and very thoroughly in Chandrasekhar (1961). When the acceleration is time-dependent, for instance when it is impulsional as when the interface is hit by a shock, one refers to the Richtmyer (1960); Meshkov (1969) instability. Modern developments include the study of the instability on time-varying (Duplat \& Villermaux 2015), and curved (Balestra et al. 2018) substrates.

The name of Taylor $(1950 \mathrm{~b})$ is associated to this instability. He indeed considered the limit with no surface tension, leading to (A 4); However his interest was more concerned with a layer of gas of a given density and finite thickness sandwiched between two gaseous phases of a different density, then accelerated perpendicular to its plane. The important case of a finite thickness layer with surface tension is envisaged below.

\section{A.2. Keller Kolodner}

Soon after the contribution of Taylor (1950b) to the subject, Keller \& Kolodner (1954) studied the case of a layer of fluid of a given density and finite thickness sandwiched between two phases of a different density, accelerated perpendicular to its plane but now incorporating an ingredient Taylor had missed in his analysis, namely surface tension. That inclusion changes the picture appreciably. 
Another way, equivalent to (A 7), to understand the selection of mode for the instability between two infinite phases is to compute an energy balance, justified in the inviscid, conservative limit. The loss of potential energy corresponds to a gain of surface energy

$$
\begin{aligned}
\Delta \rho g \lambda \int_{0}^{\xi} z \mathrm{~d} z & =\sigma\left(\sqrt{1+\xi^{\prime 2}}-1\right) \lambda, \\
\quad \text { or } \Delta \rho g \xi^{2} & \approx \sigma \xi^{\prime 2}
\end{aligned}
$$

with $\xi^{\prime} \approx \xi / \lambda$, an equilibrium which is satisfied for $\lambda^{-1} \sim k_{c}$.

When the heavy liquid is concentrated in a thin layer of thickness $h$, the above equilibrium now writes (for $\lambda>h$ )

$$
\begin{aligned}
& \Delta \rho g h \int_{0}^{\xi} z \mathrm{~d} z=\sigma\left(\sqrt{1+\xi^{\prime 2}}-1\right) \lambda, \\
& \text { or } \frac{h}{\lambda} \Delta \rho g \xi^{2} \approx \sigma \xi^{\prime 2}
\end{aligned}
$$

leading to $\lambda^{-1} \sim k_{c}^{2} h$. The wavelength of the selected mode now depends on the layer thickness $h$ and is larger for a thinner layer, unlike in the case of a film suspended on a rigid wall for which the selected mode is still given by $k_{c}$. In fact both mode selection and growth rate are affected by the layer thickness in this instability. The growth rate, obtained from (A 4 ) since $\lambda^{-1} \ll k_{c}$ is given by

$$
\frac{\ddot{\xi}}{\xi} \sim \frac{\Delta \rho}{\rho_{2}+\rho_{1}} g k_{c}^{2} h
$$

and goes to zero for an infinitely thin layer. This is because, when the two interfaces bordering the film are free to move as they are separated by a distance $h \ll k_{c}^{-1}$, their respective Laplace pressure jumps, of opposite signs, compensate, thus canceling the driving force for the longitudinal flow within the film which is thereby protected from thickness modulations. This fact, which makes the problem with surface tension very different from without, explains why, although violently accelerated when inflated, thin soap films remain stable while comparable accelerations would disrupt the interface between two infinite phases.

In the practical important case where $\rho_{2} / \rho_{1} \ll 1$ corresponding, for instance, to a liquid film (of density $\rho_{1} \equiv \rho$ ) surrounded by air, Keller \& Kolodner (1954) showed that the interfaces bordering the film can only amplify in-phase, or out-of-phase perturbations through a dispersion relation compatible with

$$
\omega^{2}=\frac{\sigma k^{3}}{\rho} \operatorname{coth}(k h)\left(1 \pm \sqrt{1-\left(1-\left(\frac{k_{c}}{k}\right)^{4}\right) \tanh ^{2}(k h)}\right)
$$

first derived in this form by Bremond \& Villermaux (2005), who also first tested its predictions experimentally for a soap film impacted by a shock wave. The dispersion relation in (A 14) transits continuously from (A 8) when $k_{c} h \gg 1$ to a shallow curve with a plateau at the level given by (A 13) when $k_{c} h \ll 1$.

The importance of this result in the dynamics of free films has been underlined by Bremond et al. (2007) and Lhuissier \& Villermaux (2009b) to understand the transverse indentations of flapping liquid sheets, by Villermaux \& Bossa (2009) for bag breakup, by Lhuissier \& Villermaux (2012b) for the stability of crumpled water bells, by Vledouts et al. (2016a) for the fragmentation of exploding liquid shells and by Klein et al. (2020) for drops hit by a laser pulse. 


\section{A.3. Helmholtz Kelvin Rayleigh}

A disturbance at the sharp interface between to parallel streams with velocities and densities $u_{1}, \rho_{1}$ and $u_{2}, \rho_{2}$ is advected at the Doppler drift velocity

$$
u_{c}=\frac{\rho_{1} u_{1}+\rho_{2} u_{2}}{\rho_{1}+\rho_{2}},
$$

intermediate between the velocities of each phases $\left(u_{1}<u_{c}<u_{2}\right)$. When a fast wind is blowing parallel to a slow liquid stream $\left(u_{1} \ll u_{2}, \rho_{2} / \rho_{1} \ll 1\right)$, the drift velocity is essentially given by that of the slow dense stream, $u_{c} \approx u_{1}$, and the intensity of the shearing velocity by that of the fast stream $u_{2}$. The instability mechanism is Bernoulli suction at the crests, and compression in the droughts

$$
\rho_{1} \lambda^{2} \ddot{\xi} \sim \rho_{2} u_{2}^{2} \frac{\xi}{\lambda} \lambda
$$

where $\xi / \lambda$ stands for the compression/dilatation factor of the distorted streamlines close to the interface. Thus

$$
\frac{\ddot{\xi}}{\xi} \sim \frac{\rho_{2}}{\rho_{1}} \frac{u_{2}^{2}}{\lambda^{2}}
$$

exhibiting an 'ultraviolet divergence' $\left(\operatorname{Re}(-i \omega) \sim k u_{2}\right)$, meaning a divergence at large $k$ which is regularized by adding the capillary restoration term $\sigma\left(\xi / \lambda^{2}\right) \lambda$ in the force balance above, ensuring the selection of mode

$$
\lambda \sim \frac{\sigma}{\rho_{2} u_{2}^{2}}
$$

The full dispersion equation incorporating also the influence of gravity is

$$
\omega=k u_{c} \pm \sqrt{\omega_{\mathrm{RT}}^{2}-\frac{\rho_{1} \rho_{2}}{\left(\rho_{1}+\rho_{2}\right)^{2}} k^{2}\left(u_{2}-u_{1}\right)^{2}}
$$

where $\omega_{\mathrm{RT}}$ is the Rayleigh-Taylor contribution given by (A 8) above. The shear $\Delta u=$ $\left|u_{2}-u_{1}\right|$ is always destabilizing. For $g=0$, the most amplified wavelength and its growth rate are $\left(\rho_{2} / \rho_{1} \ll 1\right)$

$$
\lambda \sim \frac{\sigma}{\rho_{2}(\Delta u)^{2}}, \quad \text { and } \quad \operatorname{Re}(-i \omega) \sim \frac{\rho_{2}(\Delta u)^{3}}{\sigma} \sqrt{\frac{\rho_{2}}{\rho_{1}}}
$$

The instability mechanism was first understood by von Helmholtz (1868), it was described quantitatively with the dispersion relation above by Kelvin (1871) while Lord Rayleigh (1880) complemented the analysis by considering an important ingredient in practice, namely the existence of a smooth crossover in the velocity profile between the phases: an abrupt discontinuity of velocity does not exist in nature since it is regularized by viscosity, as small as it might be. Boundary layers smoothing the velocity profiles are inevitable. When the velocity jump occurs through a transition layer of thickness $2 \delta$, we have (for $\rho_{1}=\rho_{2}$ and $u_{1}=-u_{2} \equiv u$ so that the total velocity jump is $\Delta u=2 u$ )

$$
\kappa \Omega= \pm i \sqrt{e^{-2 \kappa}-(1-\kappa)^{2}}
$$

with $\Omega=\omega /(k \Delta u)$ and $\kappa=k \delta$ (Lord Rayleigh 1880). The maximal growth rate is $0.2 u / \delta$ for $\kappa \approx 0.8$. The thickness of the crossover selects the most amplified wavelength. Starting from a true velocity discontinuity, both diffusive broadening and instability compete, altering the growth rate, and mode selection (Betchov \& Szewczyk 1963; Hinch 1984; Villermaux 1998b). The instability mechanism of this inflectional instability is however of a completely different nature than the instability of a boundary layer along a 
plate, which is driven by viscosity (Schlichting 1987). The above result was generalized to any velocities and densities between the phases by Villermaux (1998a) and applied to the stability of two-phase shear layers by Marmottant \& Villermaux (2004b). With disturbances drifting downstream as they amplify, the Kelvin-Helmholtz instability is the paradigm of 'convective instabilities' (Huerre \& Rossi 1998).

\section{A.4. Squire}

A fast liquid sheet (velocity $u_{1}$, density $\rho_{1}$ ), with thickness $h$ issuing into a lighter environment at rest $\left(u_{2}=0\right.$, density $\left.\rho_{2} \ll \rho_{1}\right)$ soon destabilizes, sustaining a flapping motion, like a flag does in the wind. The instability mechanism is of the Kelvin-Helmholtz type described above, with the additional ingredient, since the sheet has a finite thickness, that two modes of destabilization are now possible (these are actually the only two allowed modes): either the interfaces bordering the sheet grow in-phase (sinuous mode), or outof-phase (varicose mode).

(i) Sinuous mode: The Bernoulli suction mechanism operates identically to (A 16), the inertia of the liquid being now limited to a layer of thickness $h$ so that

$$
\rho_{1} h \lambda \ddot{\xi} \sim \rho_{2} u_{1}^{2} \frac{\xi}{\lambda} \lambda
$$

giving

$$
\frac{\ddot{\xi}}{\xi} \sim \frac{\rho_{2}}{\rho_{1}} \frac{u_{1}^{2}}{\lambda h}
$$

(ii) Varicose mode: The dilatational, or varicose mode implies a flow within the film, driven by the pressure difference $\Delta p \sim \rho_{2} u_{1}^{2} \xi / \lambda$ between the droughts (where it is maximal), and the crests (where it is minimal). The acceleration of the film flow is such that $\rho_{1} \partial_{t} u \sim \Delta p / \lambda$ while mass conservation writes $h u \sim \lambda \dot{\xi}$. We have

$$
\frac{\ddot{\xi}}{\xi} \sim \frac{\rho_{2}}{\rho_{1}} \frac{u_{1}^{2}}{\lambda h}\left(\frac{h}{\lambda}\right)^{2}
$$

The growth rate of this destabilization mode is thus smaller by a factor $h / \lambda$ than the one of the sinuous mode above, which is indeed preferred.

Capillary restoration $\sigma\left(\xi / \lambda^{2}\right) \lambda$ in the force balances above selects the most amplified wavelength

$$
\lambda \sim \frac{\sigma}{\rho_{2} u_{1}^{2}}
$$

The principle of this instability was already contained in Lord Rayleigh (1880) (see also the discussion in Lamb (1932)), but Squire (1953) first conducted a lucid discussion incorporating the effect of surface tension, and the competition between the destabilization modes. With $\kappa=k h, \Omega=\omega h / u_{1}$ and $\alpha=\rho_{2} / \rho_{1}$, the dispersion relations of this instability writes

$$
\begin{aligned}
(\Omega-\kappa)^{2} \Phi\left(\frac{\kappa}{2}\right)+\alpha \Omega^{2} & =\frac{\kappa^{3}}{W e} \\
\text { with } \quad \Phi(x) & =\tanh (x) \quad \text { for the sinuous mode, } \\
\text { and } \quad \Phi(x) & =\tanh (x)^{-1} \quad \text { for the varicose mode. }
\end{aligned}
$$

The Weber number $W e=\rho_{1} u_{1}^{2} h / \sigma$ should be larger than 2 for the instability to setin, meaning that the liquid sheet velocity should be larger than the group velocity of the (stable, see Taylor (1959b) and below) sinuous waves on the sheet (Villermaux \& 
Clanet 2002). This result has been readily applied to a series of situations (York et al. 1953; Hagerty \& Shea 1955; Dombrowski \& Johns 1963), including the discussion of the instability on a spatially evolving substrate (like for a Savart sheet for which $h(r) \sim r^{-1}$ ) by Bremond et al. (2007).

In the limits $\alpha \rightarrow 0$ and $W e \rightarrow 0$, (A 26) also describes the dynamics of stable capillary waves on an immobile film in an evanescent environment (Lucassen-Reynders \& Lucassen 1969). We have $\omega^{2} \sim \sigma k^{3} / \rho_{1}$ when $k h \gg 1$ as for waves over an infinitely deep medium, and, for the sinuous mode, $\omega= \pm k v$ with $v=\sqrt{2 \sigma / \rho_{1} h}$ the Taylor-Culick velocity in (3.14) for $k h \ll 1$; the celerity of long sinuous waves on a film coincides with the recession velocity of its edge. In the same long-wave limit $k h \ll 1$, we have $\omega^{2} \sim \sigma k^{4} h / \rho_{1}$ for the varicose mode, characteristic of dispersive waves on a medium with finite depth. When $k h>1$, the group velocity $\partial_{k} \omega \sim \sqrt{\sigma k / \rho_{1}}$ is larger than the Taylor-Culick velocity, explaining why these dilatational waves are systematically found to propagate ahead from the receding front as long as they are not damped by viscosity (see Fig. 5 and Buguin et al. (1999) in dewetting films).

\section{A.5. Plateau Rayleigh Weber}

Cohesion forces, because they oppose to the formation of large interface curvatures, are usually stabilizing, a stabilization which operates at large wavenumber (i.e. $\sim k^{3}$ as in (A 8)); There is one instance however where capillary forces are destabilizing. The argument, due to Plateau (1849), is geometrical. When distorted by thickness modulations with respect to its planar geometry, a liquid slab, or sheet has its surface area increased (this is the reason why the spontaneous piercing of liquid films is such a puzzling problem, see Section 4.2). A cylinder however, when distorted by longwavelengths varicose modulations of its cross-section, lowers it surface area at constant volume. The system will naturally explore more this energetically favorable corrugated state, prelude to its fragmentation in drops.

Undulations of the jet centerline in a sinusoidal fashion will not affect its net surface area if its radius remains constant. On the other hand, modulations of the jet radius may change its surface energy. Consider a liquid cylinder of volume $\Omega=\pi h_{0}^{2} L$. The perturbation

$$
h(z, \varphi)=\langle h\rangle+\varepsilon \cos (k z) \cos (m \varphi)
$$

where $k$ is the longitudinal wavenumber and $\varphi$ the azimuthal angle, can be viewed as a Fourier mode of a given initial condition $h(z, \varphi)$. The azimuthal modulations simply correspond to a corrugation of the jet's mantle, so they always increase the surface area. All azimuthal modes $m>0$ are thus stable (Chandrasekhar 1961) and, when excited by the non-circular orifice of a jet, lead to oscillations which have been used to measure surface tension (Lord Rayleigh 1879). We thus proceed with $m=0$, for which the surface energy is given by

$$
E=2 \pi \sigma \int_{L} h \sqrt{1+h^{\prime 2}} \mathrm{~d} z
$$

The mean radius $\langle h\rangle$ has to adjust itself to ensure volume conservation

$$
\Omega=\int_{L} \pi h^{2} d z=\pi h_{0}^{2} \int_{L} d z
$$

so that

$$
\langle h\rangle=h_{0}-\frac{\varepsilon^{2}}{4 h_{0}},
$$

which is thus smaller (at order $\varepsilon^{2}$ ) than the unperturbed radius $h_{0}$. The corresponding 
difference $\Delta E=E-E_{0}$ in surface energy relative to the initial state $E_{0}=2 \pi h_{0} \sigma \int d z$ is

$$
\frac{\Delta E}{E_{0}}=\frac{\varepsilon^{2}}{4 h_{0}^{2}}\left[\left(k h_{0}\right)^{2}-1\right],
$$

showing that all modes with wavelength $\lambda=2 \pi / k$ larger than the perimeter of the jet have a negative relative energy $\Delta E<0$, and are thus potentially unstable, as concluded by Plateau (1849). To find the fastest growing wavelength, on has to wonder about the dynamics of each mode in the unstable range $0<x<1$ where $x=k h_{0}$.

The dispersion equation of this instability was first established in the inviscid limit by Lord Rayleigh (1878), followed by a study of the very viscous limit (Lord Rayleigh 1892). Chandrasekhar (1961) considered the problem comprehensively. The equations feature the necessary Bessel functions of the cylindrical geometry and in the inviscid limit, we have, with $t_{c}=\sqrt{\rho h_{0}^{3} / \sigma}$

$$
\omega^{2} t_{c}^{2}=-x\left(1-x^{2}\right) \frac{I_{1}(x)}{I_{0}(x)} .
$$

The most amplified wavelength is $\lambda \approx 9 h_{0}$, corresponding to $x \approx 0.67$. This wavelength is large compared to the cylinder radius, suggesting that a long wave one-dimensional description of the problem will be a satisfactory caricature. Weber (1931) took very wisely advantage of this remark, providing a straightforward derivation which incorporates easily viscous stresses. His result reads

$$
\begin{array}{r}
(-i \omega) t_{c}=\sqrt{\frac{1}{2}\left(x^{2}-x^{4}\right)+\frac{9}{4} O h^{2} x^{4}}-\frac{3}{2} O h x^{2} \\
\text { where } O h=\frac{t_{v}}{t_{c}}, \quad \text { with } \quad t_{v}=\frac{\eta h_{0}}{\sigma},
\end{array}
$$

whose limiting forms are

$$
\begin{aligned}
& (-i \omega) t_{v}=\frac{1}{6}\left(1-x^{2}\right), \quad \text { for } \quad \text { Oh } \gg 1 \\
& (-i \omega) t_{c}=\frac{1}{\sqrt{2}} x \sqrt{1-x^{2}}, \text { for } \quad \text { Oh } \rightarrow 0
\end{aligned}
$$

Equation (A 38) provides an excellent fit to the exact relation in (A 34).

All modes in the unstable range $(0<x<1)$ grow without propagation $(\operatorname{Im}(-i \omega)=0)$ whereas all stable modes $\left(x>1\right.$, wavelengths smaller than $\left.2 \pi h_{0}\right)$ have a zero growth rate, but travel along the cylinder, with a non-zero group velocity $\sqrt{\sigma k / \rho_{1}}$ tangent to the one of capillary waves on an infinitely deep medium (Appendix A.4). This instability along with many of its applications are reviewed in Eggers \& Villermaux (2008). Its generalization to the case of soft solids has been made by Mora et al. (2010).

Acknowledgements: I am indebted to my colleagues N. Bremond, H. Lhuissier, Ph. Marmottant and N. Vandenberghe, and to my students B. Bossa, B. Néel and A. Vledouts whose work is broadly reported in this review. A. Antkowiak, L. Bourouiba, L. Duchemin, J. Duplat, J. Eggers, H. Gelderblom, J. M. Gordillo, C. Inoue and S. Le Dizès have contributed to diversify, deepen and/or inspire some aspects of its content.

\section{REFERENCES}

Agbaglah, G. \& Deegan, R. D. 2014 Growth and instability of the liquid rim in the crown splash regime. J. Fluid Mech. 752, 485-496. 
Agrawal, D. C. \& Menon, V. J. 1992 Surface tension and evaporation: An empirical relation for water. Phys. Rev. A 46 (4), 2166-2169.

Aluedanin, A. B., Wang, C., Jetly, A. \& Thoroddsen, S. T. 2018 Experiments on the breakup of drop-impact crowns by marangoni holes. J. Fluid Mech. 844, 162-186.

Allen, R. F. 1975 The role of surface tension in splashing. J. Coll. Interface Sci. 51 (2), $350-351$.

Alpher, R. A., Bethe, H. \& Gamow, G. 1948 The origin of the elements. Physical Review $73(7), 803-804$.

Amarouchene, Y., Bonn, D., Meunier, J. \& Kellay, H. 2001 Inhibition of the finitetime singularity during droplet fission of a polymeric fluid. Phys. Rev. Letters 86 (16), $3558-3561$.

Anguelova, M. \& Barber, R. P. 1999 Spume drops produced by the wind tearing of wave crests. J. Phys. Oceanogr. 29, 1156-1165.

Antkowiak, A. 2013 Personal communication.

Antkowiak, A., Bremond, N., Dizès, S. Le \& Villermaux, E. 2007 Short-term dynamics of a density interface following an impact. J. Fluid. Mech. 577, 241-250.

Antonov, D. V., Piskunov, M. V. \& Strizhak, P. A. 2019 Explosive disintegration of two-component drops under intense conductive, convective, and radiant heating. Applied Thermal Engineering 152, 409-419.

Arecchi, F. T., Buah-Bassuah, P. K., Francini, F. \& Residori, S. 1996 Fragmentation of a drop as it falls in a lighter miscible fluid. Phys. Rev. E 54 (1), 424-429.

Ashgriz, N. 2011 Handbook of Atomization and Sprays. Springer, New York.

Ashgriz, N. \& Poo, J. Y. 1990 Coalescence and separation in binary collisions of liquid drops. J. Fluid Mech. 221, 183-204.

Attinger, D., Moore, C., Donaldson, A., Jafari, A. \& Stone, H. A. 2013 Fluid dynamics topics in bloodstain pattern analysis: Comparative review and research opportunities. Forensic Science International 231 (1-3), 375-396.

Avedisian, C. T. \& Andres, R. P. 1978 Bubble nucleation in superheated liquid-liquid emulsions. J. Colloid Interface Sci. 64 (3), 438-453.

Avila, S. R. G., Song, C. \& Ohl, C. D. 2015 Fast transient microjets induced by hemispherical cavitation bubbles. J. Fluid Mech. 767, 31-51.

Aylor, D. E. 2017 Aerial Dispersal of Pollen and Spores. American Phytopathological Society.

Azaiez, J. \& Homsy, G. M. 1994 Linear stability of free shear flow of viscoelastic liquids. J. FLuid Mech. 268, 37-69.

Balestra, G., Kofman, N., Brun, P.-T., Scheid, B. \& Gallaire, F. 2018 Threedimensional rayleigh-taylor instability under a unidirectional curved substrate. J. Fluid Mech. 837, 19-47.

Basaran, O. A., Gao, H. \& Bhat, P. P. 2013 Nonstandard inkjets. Annu. Rev. Fluid Mech. 45, 85-113.

Bayvel, L. \& Orzechowski, Z. 1993 Liquid atomization. Taylor \& Francis.

Bazant, Z. P. \& CAner, F. C. 2013 Comminution of solids caused by kinetic energy of high shear strain rate, with implications for impact, shock, and shale fracturing. Proc. Nat. Acad. Sci. USA 110, 19291-19294.

BÉnard, H. 1900 Les tourbillons cellulaires dans une nappe liquide. Rev. Gen. Sci. pures et appl. 11, 1261-71,1309-1328.

BÉNARD, H. 1901 Les tourbillons cellulaires dans une nappe liquide transportant de la chaleur par convection en régime permanent. Ann. de chim. Phys. 23, 62-144.

Bentley, W.A. 1904 Studies of raindrops and raindrop phenomena. Monthly Weather Review 10, 450-456.

Berendsen, C. W. J., Zeegers, J. C. H., Kruis, G. C. F. L., Riepen, M. \& Darhuber, A. A. 2012 Rupture of thin liquid films induced by impinging air-jets. Langmuir 28 (26), 9977-9985.

Betchov, R. \& Szewczyk, G. 1963 Stability of a shear layer between parallel streams. Phys. Fluids 6, 1391-1395.

Betterton, M. D. \& Brenner, M. P. 1999 Electrostatic edge instability of lipid membranes. Phys. Rev. Lett. 82 (7), 1598-1601. 
Betts, M. G. \& AL. 2019 Extinction filters mediate the global effects of habitat fragmentation on animals. Science 366, 1236-1239.

Bird, J. C., de Ruiter, R., Courbin, L. \& Stone, H. A. 2010 Daughter bubble cascades produced by folding of ruptured thin films. Nature 465, 759-762.

Birkhoff, G., MacDougall, D. P., Pugh, E. M. \& Taylor, G. I. 1948 Explosives with lined cavities. J. Appl. Phys. 19, 563-582.

Blanchard, D. C. 1963 The electrification of the atmosphere by particles from bubbles in the sea. Progress in Oceanography 1, 73-202.

Blanchard, D. C. \& Sysdek, L. D. 1988 Film drop production as a function of bubble size. J. Geophys. Res. 93 (C4), 3649-3654.

Bleibel, J., Dietrich, S., Dominguez, A. \& Oettel, M. 2011 Shock waves in capillary collapse of colloids: A model system for two-dimensional screened newtonian gravity. Phys. Rev. Lett. 107, 128302.

Bohr, N. 1939 Disintegration of heavy nuclei. Nature 143, 330.

Boos, W. \& Thess, A. 1999 Cascade of structures in long-wavelength marangoni instability. Phys. Fluids 11 (6), 1484-1494.

Borda, J. C. 1763 Expériences sur la résistance des fluides. Mémoires de l'Academie Royale des Sciences pp. 356-376.

Born, M. 1937 Atomic Physics. Blackie \& Son Limited, London.

Bouasse, H. 1923 Jets, tubes et canaux. Delagrave, Paris.

Bounsse, H. 1924 Capillarité. Delagrave, Paris.

Boudaoud, A., Couder, Y. \& Amar, M. Ben 1999 Self adaptation in vibrating soap films. Phys. Rev. Lett. 82 (19), 3047-50.

Bourdin, B., Francfort, G. \& Marigo, J. 2008 The variational approach to fracture. J. Elasticity 91 (1-3), 5-148.

Bourouiba, L., Dehandschoewercker, E. \& Bush, J. W. M. 2014 Violent expiratory events: on coughing and sneezing. J. Fluid Mech. 745, 537-563.

Boussinesq, J. 1869a Théories des expériences de Savart, sur la forme que prend une veine liquide après s'être choquée contre un plan circulaire I. C. R. Acad. Sci. Paris 69, 45-48.

Boussinesq, J. $1869 b$ Théories des expériences de Savart, sur la forme que prend une veine liquide après s'être choquée contre un plan circulaire II. C. R. Acad. Sci. Paris 69, 128131.

Bowen, M. \& Tilley, B. S. 2013 On self-similar thermal rupture of thin liquid sheets. Phys. Fluids 25 (102105).

Bradley, S. G. \& Stow, C. D. 1978 Collisions between liquid drops. Phil. Trans. R. Soc. Lond. A 287 (1349), 635-675.

Brasz, C. F., Bemy, A. \& Bird, J. C. 2018 Threshold for discretely self-similar satellite drop formation from a retracting liquid cone. Phys. Rev. Fluids 3, 104002.

Bremond, N., Clanet, C. \& Villermaux, E. 2007 Atomization of undulating liquid sheets. J. Fluid Mech. 585, 421-456.

Bremond, N. \& Villermaux, E. 2005 Bursting thin liquid films. J. Fluid Mech. 524, 121-130.

Bremond, N. \& Villermaux, E. 2006 Atomization by jet impact. J. Fluid Mech. 549, 273306.

Brenner, M. P., Shi, X. D. \& Nagel, S. R. 1994 Iterated instabilities during droplet fission. Phys. Rev. Letters 73 (25), 3391-3394.

Brosseau, Q. \& Vlahovska, P. M. 2017 Streaming from the equator of a drop in an external electric field. Phys. Rev. Letters 119, 034501.

Buguin, A., Vovelle, L. \& Brochard-Wyart, F. 1999 Shocks in inertial dewetting. Phys. Rev. Lett. 83 (6), 1183-1186.

Bull, L. 1904 Rupture d'un film de savon par un projectile. Institut E.-J. Marey Chronophotographic Films, Cinémathèque Française.

Buller, A. H. R. 1909-1950 Researches on Fungi ols 1-7. Longmans, Green \& Co.

Burbidge, E. M., Burbidge, G., Fowler, W. A. \& Hoyle, F. 1957 Synthesis of the elements in stars. Rev. Mod. Phys. 29 (4), 547-650.

Burrows, A. 2000 Supernovae explosions in the universe. Nature 403, 727-733. 
Burton, J. C. \& TABorek, P. 2007 Two-dimensional inviscid pinch-off: An example of selfsimilarity of the second kind. Phys. Fluids 19, 102109.

Burzynski, D. A., Roisman, I. V. \& Bansmer, S. E. 2020 On the splashing of high-speed drops impacting a dry surface. J. FLuid Mech. 892, A2.

Bush, J. W. \& Hasha, A. E. 2004 On the collision of laminar jets: fluid chains and fishbones. J. Fluid Mech. 511, 285-310.

Casteletto, V., Cantat, I., Sarker, D., Bausch, R., Bonn, D. \& Meunier, J. 2003 Stability of soap films: Hysteresis and nucleation of black films. Phys. Rev. Lett. 90, 048302-(4).

Champougny, L., Miguet, J., Henaff, R., Restagno, F., Boulogne, F. \& Rio, E. 2018 Influence of evaporation on soap film rupture. Langmuir 34, 3221-3227.

Champougny, L., Rio, E., Restagno, F. \& Scheid, B. 2017 The break-up of free films pulled out of a pure liquid bath. J. Fluid Mech. 811, 499-524.

Chandra, S. \& Avedisian, C. T. 1991 On the collision of a droplet with a solid surface. Proc. Roy. Soc. London A 432, 13-41.

Chandrasekhar, S. 1961 Hydrodynamic and hydromagnetic stability. New York: Dover publication.

Chateau, J. \& Lhuissier, H. 2019 Breakup of a particulate suspension jet. Phys. Rev. Fluids 4, 012001(R).

Chen, Y.-J. \& Steen, P. H. 1997 Dynamics of inviscid capillary breakup: collapse and pinchoff of a film bridge. J. Fluid Mech. 341, 245-267.

Chepushtanova, S. V. \& Kliakhandler, I. L. 2007 Slow rupture of viscous films between parallel needles. J. Fluid Mech. 573, 297-310.

Clanet, C. 2007 Waterbells and liquid sheets. Annu. Rev. Fluid Mech. 39, 469-496.

Clanet, C. \& Lasheras, J. 1999 Transition from dripping to jetting. J. Fluid Mech. 383, 307.

Clanet, C. \& Villermaux, E. 2002 Life of a smooth liquid sheet. J. Fluid Mech. 462, 307340.

Clarke, N. S. 1969 The asymptotic effects of surface tension and viscosity on an axiallysymmetric free jet of liquid under gravity. Quart. J. Mech. and Appl. Math 22, 247-256.

Clasen, C., Bico, J., Entov, V. \& McKinley, G. H. 2009 'gobbling drops': the jetting/dripping transition in flows of polymer solutions. J. Fluid Mech. 636, 5-40.

Clausius, R. 1862 On the application of the theorem of the equivalence of transformations to the internal work of a mass of matter. Phil. Mag. 24 (159), 81-97.

Clay, P. 1940 The mechanism of emulsion formation in turbulent flow. I. Experimental part. Proc. R. Acad. Sci. Amsterdam 43, 852-865.

Cochran, R. E. \& AL. 2017 Molecular diversity of sea spray aerosol particles: Impact of ocean biology on particle composition and hygroscopicity. Chem 2, 655-667.

Cohen, I., Li, H., Hougland, J., Mrksich, M. \& Nagel, S. R. 2001 Using selective withdrawal to coat microparticles,. Science 292, 265.

Cointe, R. \& Armand, J.-L. 1987 Hydrodynamic Impact Analysis of a Cylinder. J. Offshore Mech. Arct. Eng 109 (3), 237-243.

Cole, R. H. 1948 Underwater explosions. Princeton University Press, Princeton, New Jersey.

Cooker, M. J. \& Peregrine, D. H. 1995 Pressure-impulse theory for liquid impact problems. J. Fluid Mech. 297, 193-214.

Coulson, J. M., Richardson, J. F., Backhurst, J. R. \& Harker, J. H. 2002 Chemical Engineering, 5th edn., , vol. 2. Oxford: Butterworth-Heinemann.

Courant, R., Robbins, H. \& Stewart, I. 1996 What is Mathematics?. Oxford University Press.

Courbin, L. \& Stone, H. A. 2006 Impact, puncturing, and the self-healing of soap films. Phys. Fluids 18 (091105).

Crapper, G. D., Dombrowski, N., Jepson, W. P. \& Pyott, G. A. D. 1973 A note on the growth of kelvin-helmholtz waves on thin liquid sheets. J. Fluid Mech. 57, 671-672.

Craster, R. V. \& Matar, O. K. 2009 Dynamics and stability of thin liquid films. Rev. Mod. Phys. 81 (3), 1131-1198.

Cu, K., Bansal, R., Mitragotri, S. \& Fernadez Rivas, D. 2019 Delivery strategies for 
skin: Comparison of nanoliter jets, needles and topical solutions. Annals of Biomedical Engineering pp. https://doi.org/10.1007/s10439-019-02383-1.

Culick, F. E. C. 1960 Comments on a ruptured soap film. J. Appl. Phys. 31, 1128-1129.

Curl, R. L. 1963 Dispersed phase mixing: I. theory and effects in simple reactors. AIChE Journal 9, 175-181.

DA VincI, L. 1508 Codex Leicester. In The Notebooks of Leonardo da Vinci (ed. translated in english by MacCurdy). George Brazillier: New York.

Day, R. F., Hinch, E. J. \& Lister, J. R. 1998 Self-similar capillary pinchoff of an inviscid fluid. Phys. Rev. Letters. 80 (4), 704(4).

Dear, J. P., Field, J. E. \& Walton, A. J. 1988 Gas compression and jet formation in cavities collapsed by a shock wave. Nature 332 (6164), 505-508.

Debrégeas, G., de Gennes, P.-G. \& Brochard-Wyart, F. 1998 The life and death of "bare" viscous bubbles. Science 279, 1704-1707.

Debrégeas, G., Martin, P. \& Brochard-Wyart, F. 1995 Viscous bursting of suspended films. Phys. Rev. Lett. 75 (21), 3886-3889.

Decent, S. \& King, A. 2008 Surface-tension-driven flow in a slender cone,. IMA J. Appl. Math. 73 (37-68).

Deguen, R., Landeau, M. \& Olson, P. 2014 Turbulent metal-silicate mixing, fragmentation, and equilibration in magma oceans. Earth and Planetary Science Letters 391, 274-287.

Delaunay, Ch. 1841 Sur la surface de révolution dont la courbure moyenne est constante. Journal de mathématiques pures et appliquées 1ère série 6, 309-314.

Denkov, N. D. 2004 Mechanisms of foam destruction by oil-based antifoams. Langmuir 20 (22), 9463-9505.

Derjaguin, B. V., Churaev, N. V. \& Muller, V. M. 1987 Surface Forces. Plenum, New York.

Dimotakis, P. E. 2000 The mixing transition in turbulent flows. J. Fluid Mech 409, 69-98.

DiPersio, R., Simon, J. \& Martin, T. H. 1960 A study of jets from scaled conical shaped charge liners. Tech. Rep.. Army ballistic research lab Aberdeen proving ground MD.

Do, J. L. \& Friscic, T. 2017 Mechanochemistry: A force of synthesis. ACS Central Science 3, 13-19.

Dombrowski, N. \& Fraser, R. P. 1954 A photographic investigation into the desintegration of liquid sheets. Phil. Trans. R. Soc. Lond. A 247, 101-130.

Dombrowski, N. \& Johns, W. R. 1963 The aerodynamics instability and disintegration of viscous liquid sheets. Chem. Eng. Sci. 18, 203-214.

Doméjean, H., Bibette, J. \& Bremond, N. 2016 Traffic collision during the breakup of an aqueous viscous compound jet. Phys. Rev. Fluids 1, 063903.

Dressaire, E., Courbin, L., Delancy, A., Roper, M. \& Stone, H. A. 2013 Study of polygonal water bells: inertia-dominated thin-film flows over microtextured surfaces. $J$. Fluid Mech. 721, 46-57.

Driessen, T., Sleutel, P., Dijksman, F., Jeurissen, R. \& Lohse, D. 2014 Control of jet breakup by a superposition of two rayleigh-plateau-unstable modes. J. Fluid. Mech. 749, $275-296$.

Duchemin, L., Le Dizès, S., Vincent, L. \& Villermaux, E. 2015 Self-similar impulsive capillary waves on a ligament. Phys. Fluids 27, 051704.

Duguid, J. P. 1946 The size and the duration of air-carriage of respiratory droplets and dropletnuclei. J. Hyg. (Lond) 6, 471-479.

Duplat, J. \& Villermaux, E. 2015 Luminescence from collapsing centimeter bubbles expanded by chemical reaction. Phys. Rev. Letters 115, 094501.

Dupré, A. 1867 Théorie mécanique de la chaleur. Ann. de chim. Phys. 11, 194.

Dupré, A. 1869 Théorie mécanique de la chaleur. Gauthiers-Villars, Paris.

Edgerton, H. E. 1979 Moments of Vision: The Stroboscopic Revoltion in Photography. MIT Press.

Edwards, D. A., Hanes, J., Caponetti, G., Hrkach, J., Ben-Jebria, A., Eskew, M. L., Mintzes, J., Deaver, D., Lotan, N. \& Langer, R. 1997 Large porous particles for pulmonary drug delivery. Science 276 (5320), 1868-1872.

EgGers, J. 1993 Universal pinching of 3d axisymmetric free surface flow. Phys. Rev. Letters 71 (21), 3458-3460. 
EGGers, J. 1997 Nonlinear dynamics and breakup of free-surface flows. Rev. Mod. Phys. 69, 865-929.

Eggers, J. \& Villermaux, E. 2008 Physics of liquid jets. Rep. Prog. Phys. 71, 36601.

Eisenklam, P. 1964 On ligament formation from spinning discs and cups. AICHE J. 19 (9), 693-694.

Erneux, T. \& Davis, S. H. 1993 Nonlinear rupture of free films. Phys. Fluids A 5 (5), 11171122.

Falcon, E., Laroche, C. \& Fauve, S. 2007 Observation of gravity-capillary wave turbulence. Phys. Rev. Letters. 98, 094503.

Fineberg, J. \& Marder, M. 1999 Instability in dynamic fracture. Physics Reports-Review Section Of Physics Letters 313 (1-2), 1-108.

Flores-Kim, J., S., Dobihal G., Fenton, A., Rudner, D. Z. \& Bernhardt, T. G. 2019 A switch in surface polymer biogenesis triggers growth-phase-dependent and antibioticinduced bacteriolysis. eLife 8, e44912.

FLÜGGE, C. 1897 Ueber luftinfection. Zeitschrft fur Hygiene und Infektionskrankheite 25, 179224.

Frankel, I. \& Weihs, D. 1985 Stability of a capillary jet with linearly increasing axial velocity (with application to shaped charges). J. Fluid Mech. 155, 289-307.

Frankel, I. \& Weins, D. 1987 Influence of viscosity on the capillary instability of a stretching jet. J. Fluid Mech. 185, 361-383.

Frankel, S. \& Mysels, K. 1969 The bursting of soap films. II. Theorical considerations. J. Phys. Chem. 73 (9), 3028-3038.

Frens, G. 1974 Aerodynamic drag on bursting bubbles. J. Phys. Chem. 78 (19), 1949-53.

Friedlander, S K 2000 Smoke, Dust and Haze, 2nd edn. Oxford, UK: Oxford University Press.

Frost, D. L. 1988 Dynamics of explosive boiling of a droplet. Phys. Fluids 31 (9), 2554-2561.

Frost, D. L., Ornthanalai, C., Zarei, Z., Tanguay, V. \& Zhang, F. 2007 Particle momentum effects from the detonation of heterogeneous explosives. J. Appl. Phys. 101, 113529.

Gañán-Calvo, A. M. 1997 Cone-jet analytical extension of taylor's electrostatic solution and the asymptotic universal scaling laws in electrospraying. Phys. Rev. Lett. 79, 217.

Ganan-Calvo, A. M. 1998 Generation of steady liquid microthreads and micro-sized monodisperse sprays in gas sreams. Phys. Rev. Lett. 80 (2), 285-288.

Ganan-Calvo, A. M. \& Gordillo, J. M. 2001 Perfectly monodisperse microbubbling by capillary flow focussing. Phys. Rev. Lett. 87 (27), 274501(4).

Garrett, P. R. 1992 Defoaming: Theory and Industrial Applications. Surfactant Science Series 45. Taylor \& Francis.

Gekle, S. \& Gordillo, J. M. 2010 Generation and breakup of worthington jets after cavity collapse. part 1. jet formation. J. Fluid Mech. 663, 293-330.

Gelderblom, H., Lhuissier, H., Klein, A. L., Bouwhuis, W., Lohse, D., Villermaux, E. \& Snoeiser, J. H. 2016 Drop deformation by laser-pulse impact. J. Fluid Mech. 794, 676-699.

De Gennes, P.-G. 1985 Wetting: Statics and dynamics. Rev. Mod. Phys. 57 (3), 827-863.

DE Gennes, P.-G. 1996 Mechanics of soft interfaces. Faraday Discuss. 104, 1-8.

De Gennes, P.-G. 1998 Progression d'un agent de coalescence dans une emulsion. C. R. Acad. Sci. Paris Série IIb 326, 331-335.

de Gennes, P.-G., Brochard-Wyart, F. \& Quere, D. 2004 Capillarity and Wetting Phenomena. Springer-Verlag, New York.

Ghabache, E., Antkowiak, A., Josserand, C. \& SÉon, T. $2014 a$ On the physics of fizziness: How bubble bursting controls droplets ejection. Physics of Fluids 26 (12), 121701.

Ghabache, É. \& SÉon, T. 2016 Size of the top jet drop produced by bubble bursting. Phys. Rev. Fluids 1 (5), 051901(R).

Ghabache, É., Seon, T. \& Antkowiak, A. $2014 b$ Liquid jet eruption from hollow relaxation. J. Fluid. Mech. 761, 206-219.

Gilet, T. \& Bourouiba, L. 2015 Fluid fragmentation shapes rain-induced foliar disease transmission. J. R. Soc. Interface 12 (20141092). 
Gladden, J.R., Handzy, N.Z., Belmonte, A. \& Villermaux, E. 2005 Dynamic buckling and fragmentation in brittle rods. Phys. Rev. Letters 94, 035503.

Glantz, S. A. \& Bareham, D. W. 2018 E-cigarettes: Use, effects on smoking, risks, and policy implications. Annu. Rev. Public Health 39, 215-35.

Gonnermann, H. M. 2005 Magma fragmentation. Annu. Rev. Earth Planet. Sci. 43, 431-458.

Goodridge, C. L., Tao Shi, W. \& Lathrop, D. 1996 Threshold dynamics of singular gravitycapillary waves. Phys. Rev. Letters. 76 (11), 1824-1827.

Gordillo, J. M., Lhuissier, H. \& Villermaux, E. 2014 On the cusps bordering liquid sheets. J. Fluid Mech. 754, R1.

Gordillo, J. M. \& PÉrez-SABorid, M. 2005 Aerodynamic effects in the breakup of liquid jets: on the first wind-induced breakup regime. J. Fluid Mech. 541, 1-20.

Gordillo, J. M. \& Rodríguez-Rodríguez, J. 2019 Capillary waves control the ejection of bubble bursting jets. J. Fluid. Mech. 867, 556-571.

Gordillo, L., Agbaglah, G., Duchemin, L. \& Josserand, C. 2011 Asymptotic behavior of a retracting two-dimensional fluid sheet. Phys. Fluids 23, 122101.

Grady, D. E. 1982 Local inertial effects in dynamic fragmentation. J. App. Phys. 53, 322-325.

Grady, D. E. 2006 Fragmentation of rings and shells: the legacy of N. F. Mott. Berlin: SpringerVerlag.

Grätz, S., Beyer, D., Tkachova, V., Hellmann, S., Berger, R., Feng, X. \& BORCHARDT, L. 2018a The mechanochemical scholl reaction - a solvent-free and versatile graphitization tool. Chem. Commun. 54, 5307-5310.

Grätz, S., Oltermann, M., Troschie, E., PaAsch, S., Krause, S., Brunner, E. \& BorchardT, L. $2018 b$ Solvent-free synthesis of a porous thiophene polymer by mechanochemical oxidative polymerization. J. Mater. Chem. A 6, 21901-21905.

Griffith, A. A. 1921 The phenomena of rupture and flow in solids. Phil. Trans. R. Soc. London A 221, 163-198.

Guéna, G., Poulard, C. \& Cazabat, A.-M. 2007 Evaporating drops of alkane mixtures. Colloids Surf. Physicochem. Eng. Asp. 298 (1-2), 2-11.

Hagerty, W. W. \& Shea, J. F. 1955 A study of the stability of plane fluid sheets. J. Appl. Mech. 22, 509-514.

Han, Y., Durst, F. \& Zeilmann, M. 2004 High-pressure-driven twin-jet sprays and their properties. Atomization and Sprays 24 (5), 375-401.

Hassett, M. O., Fischer, M. W. F., Sugawara, Z. T., Stolze-Rybczynski, J. \& Money, N. P. 2013 Splash and grab: Biomechanics of peridiole ejection and function of the funicular cord in bird's nest fungi. Fungal Biology 117, 708-714.

Hasson, D. \& PeCK, R. E. 1964 Thickness distribution in a sheet formed by impinging jets. AIChE J. 10, 752-754.

Hawksbee, F. 1709 Physico-mechanical experiments on various subjects. R. Brugis, London.

von Helmholtz, H. 1847 Uber die Erhaltung der Kraft, eine physikalishe Abhandlung. G. Reimer, Berlin.

von Helmholtz, H. 1868 On discontinuous movements of fluids. Philosophical magazine 36, $337-346$.

Henderson, L. F. 1989 On the refraction of shock waves. J. Fluid Mech. 198, 365-386.

Herman, J. \& Mesler, R. 1987 Bubble entrainment from bursting bubbles. Journal of Colloid and Interface Science 117 (2), 565 - 569.

Hernández-SÁnchez, J. F., Eddi, A. \& Snoeijer, J. H. 2015 Marangoni spreading due to a localized alcohol supply on a thin water film. Phys. Fluids $\mathbf{2 7}$ (032003).

Hewitt, A. J. 2000 Spray drift: impact of requirements to protect the environment. Crop Protection 19, 623-627.

Higuera, F. J., Medina, A. \& Liñán, A. 2008 Capillary rise of a liquid between two vertical plates making a small angle. Phys. Fluids 20, 102102.

Hilz, E., Vermeer, A. W. P., Cohen Stuart, M. A. \& Leermakers, F. A. M. 2012 Mechanism of perforation based on spreading properties of emulsified oils. Atomization and Sprays 22 (12), 1053-1075.

HINCH, E. J. 1984 A note on the mechanism of the instability at the interface between two shearing fluids. J. Fluid. Mech. 144, 463-465. 
Hinch, E. J. \& Saint-Jean, S. 1999 The fragmentation of a line of balls by an impact. Proc. of the Royal Soc. A: Math., Phys. and Eng. Science 455, 3201-3220.

Hinze, J. 1955 Fundamentals of the hydrodynamic mechanism of splitting in dispersion processes. AIChE J. 1 (3), 289-295.

Hirst, J. M., Stedman, O. J. \& Hogg, W. H. 1967 Long-distance spore transport: Methods of measurement, vertical spore profiles and the detection of immigrant spores. J. gen Microbiol. 48, 329-355.

Hobbs, P. V. \& Rangno, A. L. 2004 Super-large raindrops. Geophys. Res. Letters 31, L13102.

Hoepffrer, J. \& Paré, G. 2013 Recoil of a liquid filament: escape from pinch-oo through creation of a vortex ring. J. Fluid Mech. 734, 183-197.

Howard, J. L., CaO, Q. \& Browne, D. L. 2018 Mechanochemistry as an emerging tool for molecular synthesis: what can it offer? Chem. Sci 9, 3080-3094.

Howinson, S., Ockendon, D., Olivier, J. M., Purvis, R. \& Smith, F. T. 1991 Incompressible water-entry problems at small deadrise angles. J. Fluid Mech. 222, 215230.

Howland, C. J., Antkowiak, A., Castrejon-Pita, J. R., Howison, S. D., Oliver, J. M., Style, R. W. \& Castrejon-Pita, A. A. 2016 It's harder to splash on soft solids. Phys. Rev. Letters 117, 184502.

Hoyt, J. W. \& Taylor, J. 1977 Waves on water jets. J. Fluid Mech. 83, 119-.

HuAng, J. C. P. 1970 The break-up of axisymmetric liquid sheets. J. Fluid Mech. 43, 305-319.

Huerre, P. \& Rossi, M. 1998 Hydrodynamic instabilities in open flows. In Hydrodynamics and Nonlinear Instabilities (ed. C. Godrèche \& P. Manneville), pp. 81-294. Cambridge University Press.

Ilievski, F., Mani, M., Whitesides, G. M. \& Brenner, M. P. 2011 Self-assembly of magnetically interacting cubes by a turbulent fluid flow. Phys. Rev. E 83, 017301.

Ilton, M., DiMaria, C. \& Dalnoki-Veress, K. 2016 Direct measurement of the critical pore size in a model membrane. Phys. Rev. Lett. 117 (257801).

Ingold, C. T. 1971 Fungal Spores: Their liberation and dispersal. Clarendon Press-Oxford.

Inoue, C., Izato, Y., Miyake, A. \& Villermaux, E. 2017 Direct self-sustained fragmentation cascade of reactive droplets. Phys. Rev. Letters 118, 074502.

Isenberg, C. 1978 The Science of Soap Films and Soap Bubbles, 2nd edn. Dover Publications, New York.

Israelachvili, J. 1991 Intermolecular \& Surface Forces, 2nd edn. Academic Press.

JACKson, J. D. 1998 Classical Electrodynamics, 3rd edn. New York: Wiley.

Javadi, A., Eggers, J., Bonn, D., Habibi, M. \& Ribe, N. M. 2013 Delayed capillary breakup of falling viscous jets. Phys. Rev. Letters. 110 (14), 144501.

Jensen, O. E. \& Grotberg, J. B. 1992 Insoluble surfactant spreading on a thin viscous film: Shock evolution and film rupture. J. Fluid Mech. 240, 259-288.

Jensen, O. E. \& Grotberg, J. B. 1993 The spreading of heat or soluble surfactant along a thin liquid film. Phys. Fluids A 5 (1), 58-68.

Josserand, C. \& Thoroddsen, S. T. 2016 Drop Impact on a Solid Surface. Annu. Rev. Fluid Mech. 48 (1), 365-391.

JURIN, J. 1719 An account of some experiments shown before the royal society; with an enquiry into the cause of the ascent and suspension of water in capillary tubes. Phil. Trans. $R$. Soc. Lond. A 30, 739-747.

Kabova, Y. O., Alexeev, A., Gambaryan-Roisman, T. \& Stephan, P. 2006 Marangoniinduced deformation and rupture of a liquid film on a heated microstructured wall. Phys. Fluids 18 (012104).

Kalliadasis, S., Ruyer-Quil, C., Scheid, B. \& Velarde, M. G. 2012 Falling Liquid Films. Springer.

Kamat, P. M., Wagoner, B. W., Thete, S. S. \& A., Basaran O. 2018 Role of marangoni stress during breakup of surfactant-covered liquid threads: Reduced rates of thinning and microthread cascades. Phys. Rev. Fluids 3, 043602.

Kedrinskit, V. K. 2005 Hydrodynamics of Explosions. Springer, Berlin Heidelberg.

KeDRINSKII, V. K. 2009 Hydrodynamic aspects of explosive eruptions of volcanoes: simulation problems. Shock Waves 18, 451-464.

Keiser, L., Bense, H., Colinet, P., Bico, J. \& Reyssat, E. 2017 Marangoni bursting: 
Evaporation-induced emulsification of binary mixtures on a liquid layer. Phys. Rev. Letters. 118, 074504.

Keller, J. B., King, A. \& Ting, L. 1995 Blob formation. Phys. Fluids 7 (1), 226-228.

Keller, J. B. \& Kolodner, I. 1954 Instability of liquid surfaces and the formation of drops. J. Appl. Phys. 25 (7), 918-921.

Keller, J. B. \& Miksis, M. 1983 Surface tension driven flows. J. Appl. Math. 43 (2), 268-277.

Kelvin, Lord 1871 Hydrokinetic solutions and observations. Philosophical magazine 42, 362377.

Kendall, K 1978 The impossibility of comminuting small particles by compression. Nature 272, 710-711.

Keshavarz, B., Houze, E. C., Moore, J. R., Koener, M. R. \& McKinley, G. H. 2020 Rotary atomization of newtonian and viscoelastic liquids. Phys. Rev. Fluids 5, 033601.

Keshavarz, B., Houze, E. C., Moore, J. R., Koerner, M. R. \& McKinley, G. H. 2016 Ligament mediated fragmentation of viscoelastic liquids. Phys. Rev. Letters. 117, 154502.

Kim, I. \& Sirignano, W. A. 2000 Three-dimensional wave distortion and disintegration of thin planar liquid sheets. J. Fluid Mech. 410, 147-183.

Kitavtsev, G., Fontelos, M. A. \& Eggers, J. 2018 Thermal rupture of a free liquid sheet. J. Fluid Mech. 840, 555-578.

Klein, A. L., Bouwhuis, W., Visser, C. W., Lhuissier, H., Sun, C., Snoeijer, J. H., Villermaux, E., Lohse, D. \& Gelderblom, H. 2015 Drop Shaping by Laser-Pulse Impact. Phys. Rev. Appl. 3 (4), 044018.

Klein, A. L., Kurilovich, D., Lhuissier, H., Versolato, O. O., Lohse, D., Villermaux, E. \& Gelderblom, H. 2020 Drop fragmentation by laser-pulse impact. J. Fluid Mech. 893, A7.

Knelman, F. H., Dombrowski, N. \& Newitt, D. M. 1954 Mechanism of the bursting of bubbles. Nature 173, 261.

Kolmogorov, A. N. 1941a The local structure of turbulence in incompresible viscous fluid for very large Reynolds' numbers. Dokl. Akad. Nauk. SSSR 30, 301.

Kolmogorov, A. N. $1941 b$ On the logarithmic normal distribution of particles sizes under grinding. Dokl. Akad. Nauk SSSR 31, 99-101.

Kolmogorov, A. N. 1949 On the breakage of drops in a turbulent flow. Dokl. Akad. Nauk SSSR 66, 825-828.

Kooij, S., Astefanei, A., Corthals, G. L. \& Bonn, D. 2019 Size distributions of droplets produced by ultrasonic nebulizers. Scientific Reports $\mathbf{9}, 6128$.

Kooij, S., Sijs, R., Denn, M. M., Villermaux, E. \& Bonn, D. 2018 What determines the drop size in sprays? Phys. Rev. X 8, 031019.

Koros, R. M., Deckers, J. \& Boudart, M. 1960 More experiments on liquid films. J. Appl. Phys. 31, 1129-1130.

Kroto, H.W., Heath, J.R., O’Brien, S.C., Curl, R.F. \& Smalley, R.E. 1985 C 60: buckminsterfullerene. Nature 318, 162-163.

Kulkami, V. \& Sojka, P. E. 2004 Bag breakup of low viscosity drops in the presence of a continuous air jet. Phys. Fluids 26 (072103).

Lafrance, P. \& Ritter, R. C. 1977 Capillary breakup of a liquid jet with a random initial perturbation. J. Appl. Mech. 44 (3), 385-388.

Lamb, H. 1932 Hydrodynamics, 6th edition.. Cambridge University Press.

Landau, L. \& Lifshitz, E. 1987 Fluid Mechanics. Pergamon Press (Oxford).

Landeau, M., Deguen, R. \& Olson, P. 2014 Experiments on the fragmentation of a buoyant liquid volume in another liquid. J. Fluid Mech. 749, 478-518.

Lane, W. R. \& Green, H. L. 1956 The mechanics of drops and bubbles. In Survey in Mechanics (ed. G. K. Batchelor \& R. M. Davies), pp. 162-215. Cambridge University Press, Cambridge.

Laplace, P.-S. 1805 Traité de mécanique céleste, vol. IV, supplément au livre X: Sur l'action capillaire, pp. 1-65. Courcier, Paris.

Lavoisier, A. L. 1789 Traîté Élémentaire de Chimie. Cuchet, Paris.

Lavrentiev, M. \& Chabat, B. 1980 Effets hydrodynamiques et modèles mathématiques. Éditions MIR, translated from the 1977 russian edition. 
Lawn, B. R. 1993 Fracture of brittle solids - Second edition. Cambridge, UK: Cambridge Univ Press.

Layes, G., Jourdan, G. \& Houns, L. 2003 Distortion of a spherical gaseous interface accelerated by a plane shock wave. Phys. Rev. Letters 91 (17), 174502-1-4.

Le DizÈs, S. 1997 Global modes in falling capillary jets. Eur. J. Mech. B/Fluids 16 (6), 761-778.

Le Dizès, S. \& Villermaux, E. 2017 Capillary jet breakup by noize amplification. J. Fluid. Mech. 810, 281-308.

Leenaars, A. F. M., Huethorst, J. A. M. \& Van Oekel, J. J. 1990 Marangoni drying: A new extremely clean drying process. Langmuir 6 (11), 1701-1703.

Lefebvre, A. H. 1989 Atomization and sprays. Hemisphere.

Lemenand, T., Valle, D. Della, Zellouf, Y. \& Peerhossaini, H. 2003 Droplets formation in turbulent mixing of two immiscible fluids in a new type of static mixer. Int. $J$. Multiphase Flow 29, 813-840.

von Lenard, P. 1904 Über regen. Meteorologische Zeitschrift 06, 92-262.

Letcher, B. H., Nislow, K. H., Coombs, J. A., O’Donnell, M. J. \& Dubreuil, T. L. 2007 Population response to habitat fragmentation in a stream-dwelling brook trout population. Plos-One 11, e1139.

Levich, V. G. \& Krylov, V. S. 1969 Surface-tension-driven phenomena. Annu. Rev. Fluid Mech. 1, 293-316.

LEwis, D. J. 1950 The instability of liquid surfaces when accelerated in a direction perpendicular to their planes. ii. Proc. R. Soc. Lond. A 202, 81-96.

Leyvraz, F. 2003 Scaling theory and exactly solved models in the kinetics of irreversible aggregation. Phys. Rep. 383, 95-212.

Lhuissier, H., Brunet, P. \& Dorbolo, S. 2016 Blowing a liquid curtain. J. Fluid Mech. 795, 784-807.

Lhuissier, H., Neel, B. \& Limat, L. 2014 Viscoelasticity breaks the symmetry of impacting jets. Phys. Rev. Letters 113, 194502.

Lhuissier, H. \& Villermaux, E. 2009a Destabilization of flapping sheets: The surprising analogue of soap films. C. R. Mécanique 337, 469-480.

Lhuissier, H. \& Villermaux, E. $2009 b$ Soap films burst like flapping flags. Phys. Rev. Lett. 103, 054501-(4).

Lhuissier, H. \& Villermaux, E. 2011 The destabilization of an initially thick liquid sheet edge. Phys. Fluids 23 (9), 091705.

Lhuissier, H. \& Villermaux, E. $2012 a$ Bursting bubble aerosols. J. Fluid Mech. 696, 5-44.

Lhuissier, H. \& Villermaux, E. $2012 b$ Crumpled water bells. J. Fluid Mech. 693, 508-540.

Lhuissier, H. \& Villermaux, E. 2013 'Effervescent' atomization in two dimensions. J. Fluid Mech. 714, 361-392.

Limat, L. 1993 Instabilité d'un liquide suspendu sous un surplomb solide : influence de l'épaisseur de la couche. C. R. Acad. Sci. II 317, 563-568.

van Limbeek, M. A. J., Lhuissier, H., Prosperetti, A., Sun, C., \& Lohse, D. 2013 Explosive boiling? Phys. Fluids 25, 091102.

Lord Rayleigh, J. W. 1878 On the instability of jets. Proc. R. Soc. Lond. A 10, 4-13.

Lord Rayleigh, J. W. 1879 On the capillary phenomena of jets. Proc. R. Soc. Lond. A 29, 71-97.

Lord Rayleigh, J. W. 1880 On the stability, or instability of certain fluid motion. Proc. Lond. Math. Soc. 11, 57.

LORD RAYLEIGH, J. W. 1883 Investigation on the character of the equilibrium of an incompressible heavy fluid of variable density. Proc. R. Soc. Lond. A 14, 170-177.

Lord Rayleigh, J. W. 1890 On the theory of surface forces. Phil. Mag. XXX, 285-298.

Lord Rayleigh, J. W. 1891 Some applications of photography. Nature 44, 249-254.

Lord RAYleigh, J. W. 1892 On the instability of a cylindre of viscous liquid under capillary forces. Phil. Mag. 34 (207), 145-155.

Lucassen-Reynders, E.H. \& Lucassen, J. 1969 Properties of capillary waves. Adv. Colloid and Interface Sci. 2, 347-395. 
Lv, C., Eigenbrod, M. \& Hard, S. 2018 Stability and collapse of holes in liquid layers. J. Fluid Mech. 855, 1130-1155.

Mandre, S., Mani, M. \& Brenner, M. P. 2009 Precursors to splashing of liquid droplets on a solid surface. Phys. Rev. Letters 102, 134502.

Marangoni, C. 1878 Difesa della teoria dell'elasticità superficiale dei liquidi. plasticità superficiale. Il Nuovo Cimento Ser. 3 III (3), 193-211.

Marangoni, C. \& Stefanelli, P. 1873 Monografia delle bolle liquide. Nuovo Cimento 9 (1), $236-256$.

Marble, F. E. 1964 Spacecraft Propulsion. Space Technology Summer Institute, ST-3 (NsG598), California Institute of Technology.

Marmanis, H. \& Thoroddsen, S. T. 1996 Scaling of the fingering pattern of an impacting drop. Phys. Fluids 8 (6), 1344-1346.

Marmottant, Ph. \& Villermaux, E. 2004 a Fragmentation of stretched liquid ligaments. Phys. Fluids 16, 2732-2741.

Marmottant, Ph. \& Villermaux, E. $2004 b$ On spray formation. J. Fluid Mech. 498, 73-111.

Marmottant, Ph., Villermaux, E. \& Clanet, C. 2000 Transient surface tension of an expanding liquid sheet. J. Colloid Interface Sci. 230, 29-40.

Marshall, J. S. \& Palmer, W. McK. 1948 The distribution of raindrops with size. J. Meteorol. 5, 165-166.

Mason, B. J. 1971 The Physics of Clouds. Clarendon Press-Oxford.

Matar, O. K. \& Craster, R. V. 2001 Models for marangoni drying. Phys. Fluids 13 (7), 1869-1883.

Maxwell, J. C. 1867 On the dynamical theory of gases. Phil. Trans. CLVII (I), 49-88.

Maxwell, J. C. 1875 Capillary Action, 9th edn. Encyclopedia Britannica.

Mc Arthur, R. H. \& Wilson, E. O. 1967 The theory of island biogeography. Princeton University Press.

McEntee, W. R. \& Mysels, K. 1969 a The bursting of soap films. I. An experimental study. J. Phys. Chem. 73 (9), 3018-3028.

McEntee, W. R. \& Mysels, K. J. $1969 b$ The bursting of soap films. i. an experimental study. J. Phys. Chem. 73, 3018-3028.

Mehdizadeh, N. Z., Chandra, S. \& Mostaghimi, J. 2004 Formation of fingers around the edges of a drop hitting a metal plate with high velocity. J. Fluid Mech. 510, 353-373.

Meier, G. E. A., Klöpper, A. \& Grabitz, G. 1992 The influence of kinematic waves on jet break down. Exp. in Fluids 12, 173-180.

Meshkov, E. E. 1969 Instability of the interface of two gases accelerated by a shock wave. Sov. Fluid Dyn. 4 (5), 151-157.

Miguet, J., Pasquet, M., Rouyer, F., Fang, Y. \& E., Rio 2020 Stability of big surface bubbles: Impact of evaporation and bubbles size. Soft Matter 16 (4), 1082-1090.

Mora, S., Phou, T., Fromental, J. M., Pismen, L. M. \& Pomeau, Y. 2010 Capillarity driven instability of a soft solid. Phys. Rev. Letters 105, 214301.

Morawska, L., Johnson, G. R., Ristovski, Z. D., Hargreaves, M., Mengersen, K., Corbett, S., Chao, C. Y. H., Li, Y. \& Katoshevski, D. 2009 Size distribution and sites of origin of droplets expelled from the human respiratory tract during expiratory activities. Aerosol science 40, 256-269.

Motт, N. F. 1947 Fragmentation of shell cases. Proc. R. Soc. London A 189, 300-308.

Moulinet, S. \& Adda-Bedia, M. 2015 Popping balloons: A case study of dynamical fragmentation. Phys. Rev. Letters 115, 184301.

Mundo, C., Sommerfeld, M. \& Tropea, C. 1995 Droplet-wall collisions: Experimental studies of the deformation and breakup process. Int. J. Multiphase Flow 21 (2), 151-173.

Mysels, K. \& Vijayendran, B. R. 1973 Film bursting. V. The effect of various atmospheres and the anomaly of newton black films. J. Phys. Chem. 77 (13), 1692-94.

Néel, B., Lhuissier, H. \& Villermaux, E. 2020 'Fines' from the collision of liquid rims. J. Fluid Mech. 893, A 16.

Néel, B. \& Villermaux, E. 2018 The spontaneous puncture of thick liquid films. J. Fluid Mech. 838, 192-221.

Newton, I. 1687 Principia Mathematica. London. 
Newton, I. 1704 Opticks. London.

Nierstrasz, V. A. \& Frens, G. 1998 Marginal regeneration in thin vertical liquid films. J. Colloid Interface Sci. 207 (2), 209-217.

Noblin, X., Yang, S. \& Dumais, J. 2009 Surface tension propulsion of fungal spores. J. Exp. Biology 212, 2835-2843.

O'Dowd, C. \& De Leeuw, G. 2007 Marine aerosol production: a review of the current knowledge. Philos. Trans. R. Soc. A 365, 1753-1774.

Oliveira, M.S.N. \& McKinley, G.H. 2005 Iterated stretching and multiple beads-on-astring phenomena in dilute solutions of high extensible flexible polymers. Phys. Fluids 17, 071704 .

Pandit, A. B. \& Davidson, J. F. 1990 Hydrodynamics of the rupture of thin liquid films. J. Fluid Mech. 212, 11-24.

Pasteur, L. 1861 Mémoire sur les corpuscules organisés qui existent dans l'atmosphère. Annales des Sciences Naturelles (Partie Zoologique) Série 4, 5-98.

Pearson, J. R. A. 1958 On convection cells induced by surface tension. J. Fluid Mech. 4 (5), 489-500.

Peregrine, D.H., Shoker, G. \& Symon, A. 1990 The bifurcation of liquid bridges. J. Fluid Mech. 212, 25-39.

Philippi, J., Lagrée, P.-Y. \& Antkowiak, A. 2016 Drop impact on a solid surface: Shorttime self-similarity. J. Fluid Mech. 795, 96-135.

Pietsch, R. B., Grothe, H., Hanlon, R., Powers, C. W., Jung, S., Ross, S. D. \& III, D. G. Schmale 2018 Wind-driven spume droplet production and the transport of pseudomonas syringae from aquatic environments. PeerJ , (DOI 10.7717/peerj.5663), $1-26$.

Planck, M. 1901 On the law of distribution of energy in the normal spectrum. Annalen der Physik 4 (3), 553-563.

Plateau, J. 1849 Recherches expérimentales et théoriques sur les figures d'équilibre d'une masse liquide sans pesanteur. Mémoires De L'académie Royale Des Sciences, Des Lettres Et Des Beaux-Arts De Belgique XXIII, 5-150.

Plateau, J. 1873 Satique expérimentale et théorique des liquides soumis aux seules forces moléculaires. Ghauthier-Villard, Paris.

Pomeau, Y. \& Villermaux, E. 2006 Two hundred years of capillarity research. Phys. Today $59(3), 39-44$.

Poulain, S. \& Bourouiba, L. 2018 Biosurfactants change the thinning of contaminated bubbles at bacteria-laden water interfaces. Phys. Rev. Lett. 121, 204502.

Poulain, S., Villermaux, E. \& Bourouiba, L. 2018 Ageing and burst of surface bubbles. J. Fluid Mech. 851, 636-671.

Poulter, T. C. \& CAldwell, B. M. 1957 The development of shaped charges for oil well completion. Petroleum Transactions, AIME 210, 11-18.

Pugh, E. M., Eichelberger, J. \& Rostocker, N. 1952 Theory of jet formation by charges with lined conical cavities. J. Appl. Phys. 23 (5), 532-536.

QIAN, J. \& LAW, C. K. 1997 Regimes of coalescence and separation in droplet collision. J. Fluid Mech. 331, 59-80.

Rallison, J. M. \& Hinch, E. J. 1995 Instability of a high-speed submerged elastic jet. J. Fluid Mech. 288, 311-324.

Ranger, A., A. \& Nicholls, J., A. 1969 Aerodynamic shattering of liquid drops. AIAA Journal 7 (2), 285-290.

Ranz, W. E. 1959 Some experiments on the dynamics of liquid films. J. Appl. Phys 30, 19501955.

RAO, C. K. \& BASU, S. 2020 Atomization modes for levitating emulsified droplets undergoing phase change. Exp. in Fluids 61, 41.

RaO, C. K., Kamakar, S. \& Basu, S. 2017 Atomization characteristics and instabilities in the combustion of multi-component fuel droplets with high volatility differential. Scientific Reports 7, 8925 .

Raufaste, C., Celestini, F., Barzyk, A. \& Frisch, T. 2015 Hole growth dynamics in a two dimensional leidenfrost droplet. Phys. Fluids 27 (3), 031704. 
Redor, I., Barthélemy, E., Michallet, H., Onorato, M. \& Mordant, N. 2019 Experimental evidence of a hydrodynamic soliton gas. Phys. Rev. Letters. 122, 214502.

Reiter, G. 1992 Dewetting of thin polymer films. Phys. Rev. Lett. 68 (1), 75-78.

Resch, F. \& Afeti, G. 1991 Film drop distribution from bubbles bursting in seawater. $J$. Geophys. Res. 96 (C6), 10681-10688.

Reyssat, E. \& Quere, D. 2006 Bursting of a fluid film in a viscous environment. EPL 76, $236-242$.

Riboux, G. \& Gordillo, J. M. 2014 Experiments of Drops Impacting a Smooth Solid Surface: A Model of the Critical Impact Speed for Drop Splashing. Phys. Rev. Lett. 113 (2), 024507.

Riboux, G. \& Gordillo, J. M. 2015 The diameters and velocities of the droplets ejected after splashing. J. Fluid Mech. 772, 630-648.

Richtmyer, R. D. 1960 Taylor instability in shock acceleration of compressible fluids. Commun. Pure Appl. Maths 13, 297-319.

von Rittinger, P. R. 1867 Lehrbuch der Aufbereitungskunde : in ihrer neuesten Entwicklung und Ausbildung systematisch dargestellt. Ernst und Korn, Berlin.

Robinson, N. D. \& Steen, P. H. 2001 Observation of singularity formation during the capillary collapse and bubble pinch-off of a soap film bridge. J. Colloid Interface Sci. 241, 448-458.

Roché, M., Li, Z., Griffiths, I. M., Le Roux, S., Cantat, I., Saint-Jalmes, A. \& Stone, H. A. 2014 Marangoni flow of soluble amphiphiles. Phys. Rev. Lett. 112 (208302).

Roisman, I. V. 2004 Dynamics of inertia dominated binary drop collisions. Phys. Fluids 16 (9), $3438-3449$.

Roisman, I. V., Horvat, K. \& Tropea, C. 2006 Spray impact: rim transverse instability initiating fingering and splash, and description of a secondary spray. Phys. Fluids 18, 102104.

Roisman, I. V., Riobo, R. \& Tropea, C. 2002 Normal impact of a liquid drop on a dry surface: model for spreading and receding. Proc. Roy. Soc. Lond. A 458, 1411-1430.

Rowlinson, J. S. 2002 Cohesion. Cambridge University Press.

Rozhkov, A., Prunet-Foch, B. \& Vignes-Adler, M. 2002 Impact of water drops on small targets. Phys. Fluids 14, 3485-3501.

Rozhkov, A., Prunet-Foch, B. \& Vignes-Adler, M. 2010 Impact of drops of surfactant solutions on small targets. Proc. R. Soc. Lond. A 466 (2122), 2897-2916.

Rozhkov, A., Prunet-Foch, B. \& Vignes-Adler, M. 2015 Star-like breakup of polymeric drops in electrical field. J. Non Netonian Mechanics 226, 46-59.

Sallam, K. A., Aalburg, C. \& Faeth, G. M. 2004 Breakup of round nonturbulent liquid jets in gaseous crossflow. AIAA Journal 42 (12), 2529-2540.

Sauter, U. S. \& Buggish, H. W. 2005 Stability of initially slow viscous jets driven by gravity. J. Fluid Mech. 533, 237-57.

SAVART, F. 1833a Mémoire sur la constitution des veines liquides lancées par des orifices circulaires en mince paroi. Annales de Chimie et de Physique 53, 337-386.

Savart, F. $1833 b$ Mémoire sur le choc de deux veines liquides animées de mouvements directement opposés. Annales de Chimie et de Physique 55, 257-310.

SAvart, F. 1833c Mémoire sur le choc d'une veine liquide lancée contre un plan circulaire. Annales de Chimie et de Physique 54, 56-87.

SAvart, F. 1833d Suite du mémoire sur le choc d'une veine liquide lancée contre un plan circulaire. Annales de Chimie et de Physique 54, 113-145.

Savva, N. \& Bush, J. W. 2009 Viscous sheet retraction. J. Fluid Mech. 626, 211-240.

Scharfman, B. E., Techet, A. H., Bush, J. W. M. \& Bourouiba, L. 2016 Visualization of sneeze ejecta: steps of fluid fragmentation leading to respiratory droplets. Exp. in Fluids $57(24), 1-9$.

Schlichting, H. 1987 Boundary layer theory, 7th edn. New York: McGraw-Hill.

Schulkes, R. M. S. M. 1996 The contraction of liquid filaments. J. Fluid Mech. 309, 277-300. Schweitzer, P. H. 1937 Mechanism of disintegration of liquid jets. J. App. Phys. 8, 513-521. Scriven, L. E. \& Sternling, C. V. 1960 The marangoni effects. Nature 187 (4733), 186-188. SEdov, L. I. 1946 Le mouvement d'air en cas d'une forte explosion. C. R. Acad. Sci. URSS 52, 17-20. 
Senchenko, S. \& Bohr, T. 2005 Shape and stability of a viscous thread. Phys. Rev. E 71 (056301).

Sharma, A. \& Reiter, G. 1996 Instability of thin polymer films on coated substrates: Rupture, dewetting, and drop formation. J. Colloid Interface Sci. 178 (2), 383-399.

Sierou, A. \& Lister, J. R. 2004 Self-similar recoil of inviscid drops. Phys. Fluids 16 (5), $1379-1394$.

Simmons, H. C. $1977 a$ The correlation of drop-sizes distributions in fuel nozzles sprays. part i. J. Eng. for Power 7, 309-314.

Simmons, H. C. $1977 b$ The correlation of drop-sizes distributions in fuel nozzles sprays. part ii. J. Eng. for Power 7, 315-319.

Smoluchowski, M. von 1917 Versuch einer mathematischen Theorie der Koagulationskinetik kolloider Lösungen. Z. Phys. Chem 92, 129-168.

Sonder, I., Harp, A. G., Graettinger, A. H., Moitra, P., Valentine, G. A., Büttner, R. \& Zimanowski, B. 2018 Meter-scale experiments on magma-water interaction. JGR: Solid Earth 123, 10597-10615.

Soundar Jerome, J. J., Vandenberghe, N. \& Forterre, Y. 2016 Unifying impacts in granular matter from quicksand to cornstarch. Phys. Rev. Letters 117, 098003.

Sovani, S.D., Sojka, P.E. \& Lefebvre, A.H. 2001 Effervescent atomization. Prog. Energy Combust. Sci. 27, 483-521.

SpIEL, D. E. 1994 The number and size of jet drops produced by air bubbles bursting on a fresh water surface. J. Geophys. Res. 99 (C4), 10289-10296.

Spiel, D. E. 1998 On the birth of film drops from bubbles bursting on seawater surfaces. $J$. Geophys. Res. 103 (C11), 24907-24918.

Squire, H. B. 1953 Investigation of the stability of a moving liquid film. Br. J. of Appl. Phys. 4, 167-169.

Stone, H. A. \& Leal, L. G. 1989 Relaxation and breakup of an initially extended drop in an otherwise quiescent fluid. J. Fluid Mech. 198, 399-427.

Stow, C. D. \& Stainer, R.D. 1977 The physical products of a splashing water drop. J. Met. Soc. Japan 55 (5), 518-531.

Sultanov, F. M. \& Yarin, A. L. 1990 Droplet size distribution in a percolation model for explosive dispersal. J. Appl. Mech. and Tech. Phys. 31 (5), 708-713.

Swanson, J. G. \& LAngefeld, O. 2015 Fundamental research in water spray systems for dust control. Transactions of the Institution of Mining and Metallurgy, Section A: Mining Technology 124 (2), 78-82.

Tagawa, Y. \& Al. 2012 Highly focused supersonic microjets. Phys. Rev. X 2, 031002.

TAKACs, L. 2013 The historical development of mechanochemistry. Chem. Soc. Rev. 42, 76497659.

TAYlor, A. M. K. P. 2008 Science review of internal combustion engines. Energy Policy 36, 4657-4667.

TAYLOR, B. 1712 (part of a letter) concerning the ascent of water between two glass planes. Phil. Trans. Roy. Soc. 27, 538.

Taylor, G. I. 1935 Statistical theory of turbulence, part i. Proc. Roy. Soc. A CLI, 421-444.

TAYLOR, G. I. $1950 a$ The formation of a blast wave by a very intense explosion. ii. the atomic explosion of 1945. Proc. R. Soc. London A 201, 175-186.

TAYLOR, G. I. $1950 b$ The instability of liquid surfaces when accelerated in a direction perpendicular to their planes. i. Proc. R. Soc. Lond. A 201 (1065), 192-196.

TAYlor, G. I. $1959 a$ The dynamics of thin sheets of fluid I. Water bells. Proc. R. Soc. Lond. A 253, 289-295.

TAYlor, G. I. $1959 b$ The dynamics of thin sheets of fluid II. Waves on fluid sheets. Proc. $R$. Soc. Lond. A 253, 296-312.

TAYlor, G. I. 1959c The dynamics of thin sheets of fluid III. Desintegration of fluid sheets. Proc. R. Soc. Lond. A 253, 313-321.

Taylor, G. I. \& Michael, D. H. 1973 On making holes in a sheet of fluid. J. Fluid Mech. $\mathbf{5 8}(4), 625-639$.

Thereulaz, G., Bonabeau, E., Saumens, C., Deneubourg, J. L., Lioni, A., Libert, F., Passera, L. \& Solé, R. 2001 Model of droplet dynamics in the argentine ant linepithema humile (mayr). Bulletin of Mathematical Biology 63, 1079-1093. 
Thomson, J. J. \& Newall, H. F. 1885 On the formation of vortex rings by drops falling into liquids, and some allied phenomena. Proc. Roy. Soc. London 39, 417-436.

Thoroddsen, S. T. 2002 The ejecta sheet generated by the impact of a drop. J. Fluid Mech. 451, 373-381.

Thoroddsen, S. T., Етон, T. G. \& Takehara, K. 2006 Crown breakup by marangoni instability. J. Fluid Mech. 557, 63-72.

Thoroddsen, S. T. \& Takehara, K. 2000 The coalescence cascade of a drop. Phys. Fluids $12(6), 1265-1267$.

Thoroddsen, S. T., Takehara, K. \& Etoh, T. G. $2012 a$ Microsplashing by drop impacts. J. Fluid Mech. 706, 560-570.

Thoroddsen, S. T., Thoraval, M.-J., Takehara, K. \& Etoh, T. G. $2012 b$ Micro-bubble morphologies following drop impacts onto a pool surface. J. Fluid Mech. 708, 469-479.

TJahjadi, M. \& Ottino, J. M. 1991 Stretching and breakup of droplets in chaotic flows. J. Fluid Mech. 232, 191-219.

Tjahjadi, M., Stone, H. A. \& Ottino, J. M. 1992 Satellite and subsatellite formation in capillary breakup. J. Fluid Mech. 243, 297-317.

TомотікA, S. 1936 Breaking up of a drop of viscous liquid immersed in another viscous fluid which is extending at a uniform rate. Proc. R. Soc. London. A 153 (879), 302-318.

Turner, C. E., Jennison, M. W. \& Edgerton, H. E. 1941 Public health applications of high-speed photography. American journal of public health 31, 319-324.

Ukiwe, C. \& Kwok, D. Y. 2005 On the maximum spreading diameter of impacting droplets on well-prepared solid surfaces. Langmuir 21, 666-673.

Utada, A. S., Lorenceau, E., Link, D. R., Kaplan, P. D., Stone, H. A. \& Weitz, D. A. 2005 Monodisperse double emulsions generated from a microcapillary device. Science 308 (5721), 537-541.

Vanhook, S. J., Schatz, M. F., Swift, J. B., McCormick, W. D. \& Swinney, H. L. 1997 Long-wavelength surface-tension-driven bénard convection: Experiment and theory. J. Fluid Mech. 345, 45-78.

Vermorel, R., Vandenberghe, N. \& Villermaux, E. 2007 Rubber band recoil. Proc. Roy. Soc. London A 463, 641-658.

Vernay, C., Ramos, L. \& Ligoure, C. 2015 a Bursting of dilute emulsion-based liquid sheets driven by a marangoni effect. Phys. Rev. Letters 115, 198302.

Vernay, C., Ramos, L. \& Ligoure, C. $2015 b$ Free radially expanding liquid sheet in air: time- and space-resolved measurement of the thickness field. J. Fluid. Mech. 764, 428-444.

Veron, F. 2015 Ocean spray. Annu. Rev. Fluid Mech. 47, 507-538.

Veron, F., Hopkins, C., Harrison, E. L. \& Mueller, J. A. 2012 Sea spray spume droplet production in high wind speeds. Geophys. Res. Letters 39, L16602.

Vielle, P. 1900 Rôle des discontinuités dans la propagation des phénomènes explosifs. $C . R$. Acad. Sci. Paris CXXLI, 413-416.

Villermaux, E. 1994 Pulsed dynamics of fountains. Nature 371, 24-25.

Villermaux, E. 1998a Mixing and spray formation in coaxial jets. J. Prop. and Power 14, $807-817$.

Villermaux, E. $1998 b$ On the role of viscosity in shear instability. Phys. Fluids 10 (2), 368-73.

Villermaux, E. 2007 Fragmentation. Annu. Rev. Fluid Mech. 39, 419-446.

Villermaux, E. 2009 Hesitant nature. J. Fluid Mech. 636, 1-4.

Villermaux, E. 2012 The formation of filamentary structures from molten silicates : Pele's hair, angel hair, and blown clinker. Comptes Rendus Mecanique 340 (8), 555-564.

Villermaux, E. 2019 Mixing versus stirring. Annu. Rev. Fluid Mech. 51, 245-273.

Villermaux, E. \& Almarcha, C. 2016 Node dynamics and cusps size distribution at the border of liquid sheets. Phys. Rev. Fluids 1 (041902).

Villermaux, E. \& Bossa, B. 2009 Single drop fragmentation determines size distribution of raindrops. Nature Physics 5, 697-702.

Villermaux, E. \& Bossa, B. 2010 Size distribution of raindrops reply. Nature Physics 6, 232.

Villermaux, E. \& Bossa, B. 2011 Drop fragmentation on impact. J. Fluid Mech. 668, 412435. 
Villermaux, E. \& Clanet, C. 2002 Life of a flapping liquid sheet. J. Fluid Mech. 462, 342-363.

Villermaux, E. \& Duplat, J. 2003 Mixing as an aggregation process. Phys. Rev. Letters. $91(18), 184501$.

Villermaux, E. \& Eloi, F. 2011 The distribution of raindrops speeds. Geophys. Res. Letters 38, L19805.

Villermaux, E., Marmottant, Ph. \& Duplat, J. 2004 Ligament-mediated spray formation. Phys. Rev. Lett. 92 (7), 074501.

Villermaux, E., Moutte, A., Amielh, M. \& Meunier, P. 2017 Fine structure of the vapor field in evaporating dense sprays. Phys. Rev. Fluids 2, 074501.

Villermaux, E., Pistre, V \& Lhuissier, H. 2013 The viscous Savart sheet. J. Fluid Mech. 730, 607-625.

Vledouts, A., Quinard, J., Vandenberghe, N. \& Villermaux, E. 2016a Explosive fragmentation of liquid shells. J. Fluid Mech. 788, 246-273.

Vledouts, A., Vandenberghe, N. \& Villermaux, E. 2015 Fragmentation as an aggregation process. Proc. R. Soc. London A 471, 20150678.

Vledouts, A., Vandenberghe, N. \& Villermaux, E. 2016b Fragmentation as an aggregation process: the role of defects. Proc. R. Soc. A 472 (20150679).

VRIJ, A. 1966 Possible mechanism for the spontaneous rupture of thin, free liquid films. Discuss. Faraday Soc. 42, 23-33.

Vu, T. T. \& Dumouchel, C. 2018 Analysis of ligamentary atomization of highly perturbed liquid sheets. Int. J. Multiphase Flows 107, 156-167.

Wacheul, J. B., Le Bars, M., Monteux, J. \& Aurnou, J. M. 2014 Laboratory experiments on the breakup of liquid metal diapirs. Earth and Planetary Science Letters 403, 236-245.

Wagner, H. 1932 Über Stoß- und Gleitvorgänge an der Oberfläche von Flüssigkeiten. Z. Angew. Math. Mech. 12 (4), 193-215.

WANG, Y. \& BourouibA, L. 2017 Drop impact on small surface: thickness and velocity profiles of the expanding sheet in the air. J. Fluid Mech. 814, 510-534.

WANG, Y. \& Bourouiba, L. 2018 Unsteady sheet fragmentation: droplet sizes and speeds. $J$. Fluid Mech. 848, 946-967.

Wang, Y., Im, K. S. \& FezzAA, K. 2008 Similarity between the primary and secondary air-assisted liquid jet breakup mechanisms. Phys. Rev. Letters 100 (154502).

Watson, E. J. 1964 The radial spread of a liquid jet over a horizontal plane. J. Fluid Mech. 20, 481-499.

Weber, C. 1931 Zum Zerfall eines Flüssigkeitsstrahles. Z. angew. Math. U. Mech. 11 (2), $136-154$.

Wedershoven, H. M. J. M., Berendsen, C. W. J., Zeegers, J. C. H. \& Darhuber, A. A. 2015 Infrared-laser-induced thermocapillary deformation and destabilization of thin liquid films on moving substrates. Phys. Rev. Appl. 3 (024005).

Wells, F. W. 1955 Airborne contagion and air hygiene. Harvard University Press, Cambridge, MA.

Wisshoff, H. 2010 The dynamics of the piezo inkjet printhead operation. Phys. Rep. 491, $77-177$.

Wildeman, S., Sterl, S., Sun, C. \& Lohse, D. 2017 Fast dynamics of water droplets freezing from the outside in. Phys. Rev. Letters. 118, 084101.

Wilson, J. E., Grib, S. W., Ahmad, A. D., Renfro, M. W., Adams, S. A. \& Salaimeh, A. A. 2018 Study of near-cup droplet breakup of an automotive electrostatic rotary bell (esrb) atomizer using high-speed shadowgraph imaging. Coatings 8 (174), 1-17.

Winslow, C.-E. A. \& Robinson, E. A. 1910 An investigation of the extent of the bacterial pollution of the atmosphere by mouth spray. The Journal of Infectious Diseases 7 (1), $17-37$.

Wodlei, F., Sebilleau, J., Magnaudet, J. \& Pimienta, V. 2018 Marangoni-driven flowerlike patterning of an evaporating drop spreading on a liquid substrate. Nature Com. 9, 820.

Wong, D. C. Y., Simmons, M. J. H., Decent, S. P., Parau, E. I. \& King, A. C. 2004 Break-up dynamics and drop size distributions created from spiralling liquid jets. Int. J. Multiphase Flows 30, 499-520. 
Woodcock, A. H., Kientzler, C. F., Arons, A. B. \& Blanchard, D. C. 1953 Giant condensation nuclei from bursting bubbles. Nature 172, 1144-45.

Worthington, A. M. 1876 On the forms assumed by drops of liquids falling vertically on a horizontal plate. Proc. Royal Soc. Lond. 25, 261-272.

Worthington, A. M. 1908 A Study of Splashes. Longmans, Green \& Co.

Wu, P. K. \& FAeth, G. M. 1995 Onset and end drop formation along the surface of turbulent jets in still gases. Phys. Fluids 7 (11), 2915-2917.

Xu, L., Barcos, L. \& Nagel, S. R. 2007 Splashing of liquids: interplay of surrounding gas and surface roughness. Phys. Rev. E 76, 066311.

Yafetto, L. \& AL. 2008 The fastest flights in nature: High-speed spore discharge mechanisms among fungi. Plos-One $\mathbf{3}$ (9), e3237.

Yaminsky, V. V., Ohnishi, S., Vogler, E. A. \& Horn, R. G. 2010 Stability of aqueous films between bubbles. part 1 . the effect of speed on bubble coalescence in purified water and simple electrolyte solutions. Langmuir 26 (11), 8061-8074.

Yarin, A. L., Pourdeyhimi, B. \& Ramakrishna, S. 2014 Fundamentals and Applications of Micro- and Nanofibers. Cambridge University Press.

YARIN, A. L. \& Weiss, D. A. 1995 Impact of drops on solid surfaces: self-similar capillary waves, and splashing as a new type of kinematic discontinuity. J. Fluid Mech. 283, 141173.

York, J. L., Stubbs, H. E. \& TeK, M. R. 1953 The mechanism of disintegration of liquid sheets. Trans. ASME 75, 1279-1286.

Young, T. 1805 An essay on the cohesion of fluids. Proc. Roy. Soc. 95, 65-87.

Yule, A. J. \& Dunkley, J. J. 1994 Atomization of melts for powder production and spray deposition. Clarendron Press, Oxford.

Zayas, G., Chiang, M. C., Wong, E., Macdonald, F., Lange, C. F., Senthilselvan, A. \& KING, M. 2012 Cough aerosol in healthy participants : fundamental knowledge to optimize droplet-spread infectious respiratory disease management. BMC Pulmonary Medicine 12 (11), 1-11.

Zeff, B., Kleber, B., Fineberg, J. \& Lathrop, D. 2000 Singularity dynamics in curvature collapse and jet erruption on a fluid surface. Nature 403, 401-404.

Zeldovich, Y. B. \& Raizer, Y. P. 2002 Physics of Shock Waves and High-Temperature Hydrodynamic Phenomena. Dover Publications, Inc., New York.

ZhANG, H. \& RAVI-ChANDAR, K. 2007 On the dynamics of necking and fragmentation - I. Real-time and post-mortem observations in Al 6061-O. Int. J. Fract. 142 (3-4), 183-217.

ZHANG, H. \& RAVI-ChANDAR, K. 2008 On the dynamics of necking and fragmentation-II. Effect of material properties, geometrical constraints and absolute size. Int. J. Fract. $150(1-2), 3-36$.

Zhang, L., Brunet, P., Eggers, J. \& Deegan, R. 2010 Wavelength selection in the crown splash. Phys. Fluids 22, 122105.

Zhao, C., Sprittles, J. E. \& Lockerby, D. A. 2019 Revisiting the rayleigh-plateau instability for the nanoscale. J. Fluid Mech. 861, R3.

Zhaо, H., Liu, H. F., Xu, J. L. \& Li, W. F. 2011 Experimental study of drop size distribution in the bag breakup regime. Ind. Eng. Chem. Research 50 (16), 9767-9773. 